Portland State University

PDXScholar

$1-1-2011$

\title{
Attaining a Sustainable Future for Public Higher Education: The Role of Institutional Effectiveness and Resource Dependence
}

Mirela Blekic

Portland State University

Follow this and additional works at: https://pdxscholar.library.pdx.edu/open_access_etds Let us know how access to this document benefits you.

\section{Recommended Citation}

Blekic, Mirela, "Attaining a Sustainable Future for Public Higher Education: The Role of Institutional Effectiveness and Resource Dependence" (2011). Dissertations and Theses. Paper 277.

https://doi.org/10.15760/etd.277

This Dissertation is brought to you for free and open access. It has been accepted for inclusion in Dissertations and Theses by an authorized administrator of PDXScholar. Please contact us if we can make this document more accessible: pdxscholar@pdx.edu. 
Attaining a Sustainable Future for Public Higher Education: The Role of Institutional Effectiveness and Resource Dependence

by

Mirela Blekic

A dissertation submitted in partial fulfillment of the requirements for the degree of

\author{
Doctor of Philosophy \\ in \\ Public Affairs and Policy
}

\author{
Dissertation Committee: \\ Craig W. Shinn, Chair \\ Jay Kenton \\ Kathi A. Ketcheson \\ Cheryl Livneh \\ Darrell Brown
}

Portland State University

(C)2011 


\begin{abstract}
The world of today's higher education organizations is characterized by complexities brought about as a result of rapid change, economic and political turbulence, and increasing global interdependence. The complexity of the environment in which colleges and universities operate is also due in part to a need to serve multiple internal and external constituencies. In order to be more responsive to the demands of its numerous constituencies and at the same time preserve their intrinsic values, colleges and universities need to know how effective they are in what they do. This research asked: To what degree does institutional effectiveness allow public colleges and universities to operate in a sustained manner over a long period of time while meeting the needs of their constituencies? The lack of criteria about what constitutes effectiveness in higher education contributes to the lack of research in this area of organizational theory.

This research examined organizational effectiveness and its measurement in higher education environment using a survey of multiple internal and external constituencies. The purpose of the survey was to gather information regarding participants' perceptions about educational outcomes, processes, and environment in higher education organizations. In addition, given the changes in how higher education institutions are financed and the potential implications of these changes for effectiveness, this research explored the degree to which resource dependence, primarily dependence on public funding, influences the effectiveness of public colleges and universities.

To address these questions the research tested the applicability of the sustainability framework as a model of effectiveness in higher education. The study
\end{abstract}


suggests modification of the elements of the sustainability and extends the use of the concept of environment as it is defined in the sustainability framework to the concept of environment as defined in organizational theory. The sustainability framework has not been tested in this way before. The results indicate that there is promise in using the sustainability framework in this modified form and suggest that this concept is worthy of further exploration. Additionally, the study examined the role of multiple constituencies in defining effectiveness in higher education. The findings indicate that there are significant differences in perceptions of effectiveness among the groups of constituencies examined in the study.

Finally, the results suggest that sources of public funding and the amount of money institutions spend per student have an influence on some aspects of effectiveness. To examine this further, the study explores the role of the political and fiscal environment in which institutions of higher education operate and offers institutional theory as a basis to explain resource dependence in public higher education. The findings of this study contribute to the field of organizational effectiveness, aid in understanding the role that public funding plays in higher education effectiveness, and contribute to the field of organizational theory more generally. 


\section{ACKNOWLEDGMENTS}

I am indebted to a number of people for their help and support during the completion of this dissertation.

First, I express my gratitude to each of my committee members. My committee chair and adviser Dr. Craig Shinn has been with me since I entered the program, teaching me to think as a researcher, guiding me through my initial ideas and helping me to shape them into a research project that led to this dissertation. He has also been invaluable in helping me navigate the process and all the steps necessary to complete the dissertation. Dr. Kathi Ketcheson was there when I needed to explore the nuances of my future project. Kathi let me examine my interests in the area of organizational performance and effectiveness, pointed to various options and asked questions that helped me to clarify the topic I wanted to pursue. Her flexibility and guidance throughout this process is much appreciated. Dr. Cheryl Livneh was especially valuable in encouraging me to think about choices I made in regards to methods and data analysis portion of my dissertation and provided detailed feedback along the way, while Dr. Darrell Brown took the time to go over the details of my instrument, providing advice and contributing to a better overall quality of the survey. Finally, my deepest appreciation goes to Dr. Jay Kenton, who was always there to guide and support me. Jay gave numerous hours, offered countless advice and provided thoughtful insight into the world of higher education. Without his help and generosity, it would be difficult to accomplish this project in a time line I set for myself. 
Additionally, there have been many individuals at PSU, too many to name, from my friends and co-workers to my supervisors, who, in their own way, supported me in my endeavor over the course of the last five years.

Lastly, and most importantly, my gratefulness goes to my family, to my parents who have always supported me, but more importantly, taught me the value of education and instilled in me the confidence to pursue my highest aspirations, and to my sister for her unwavering support in every imaginable way. I dedicate this accomplishment to them. 
Table of Contents

PAGE

ABSTRACT $\ldots \ldots \ldots \ldots \ldots \ldots \ldots \ldots \ldots \ldots \ldots \ldots \ldots \ldots \ldots \ldots \ldots \ldots \ldots \ldots \ldots \ldots \ldots \ldots \ldots \ldots \ldots \ldots \ldots, \quad \mathrm{i}$

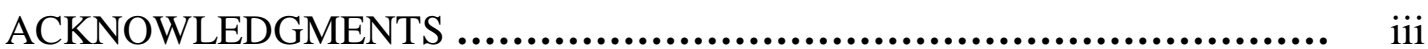

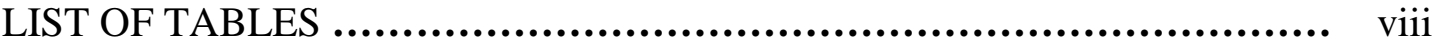

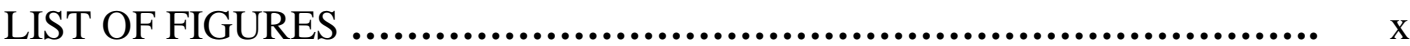

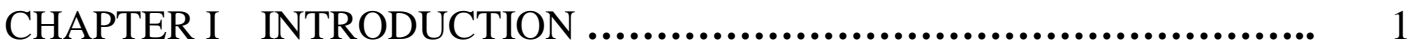

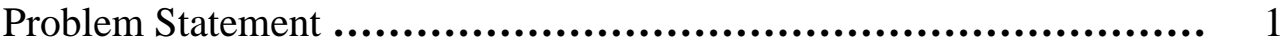

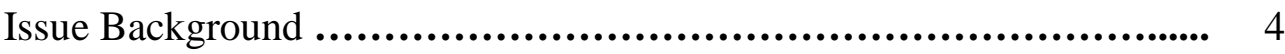

Significance of the Study $\ldots . \ldots \ldots \ldots \ldots \ldots \ldots \ldots \ldots \ldots \ldots \ldots \ldots \ldots \ldots \ldots \ldots \ldots \ldots . . . \ldots \ldots$

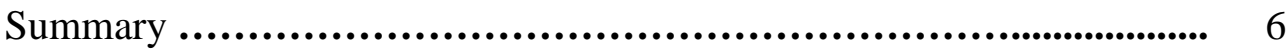

CHAPTER II LITERATURE REVIEW .................................. 8

Sustainability Framework and Its Application to Effectiveness ......... 9

Organizational Effectiveness .......................................... 13

Effectiveness in Higher Education ..................................... 19

Universities as Environmental Systems ............................... 24

Universities as Social Systems ...................................... 28

Universities as Economic Systems .................................... 31

State Appropriations vs. Tuition as Revenue Source .................... 33

Knowledge Economy as Revenue Source .............................. 37

Costs in Higher Education $. . . \ldots \ldots \ldots \ldots \ldots \ldots \ldots \ldots \ldots \ldots \ldots \ldots \ldots \ldots \ldots \ldots . . . . \ldots \ldots$ 
Effectiveness and Accountability ....................................... 41

Effectiveness and Resource Dependence .............................. 45

A Note on Perceptions .................................................. 48

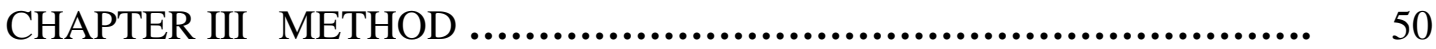

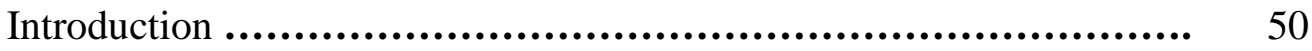

Research Model ......................................................... 52

Sample and Data Collection ............................................ 65

Method of Data Analysis ............................................... 72

Limitations of the Method ............................................... 74

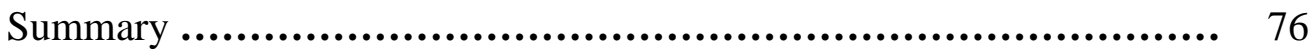

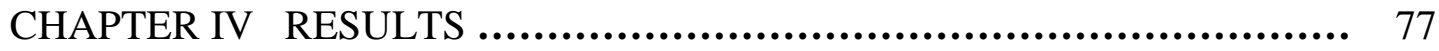

Factor Analysis .................................................... 78

One-Way Analysis of Variance ......................................... 85

Two-Way Analysis of Variance ......................................... 95

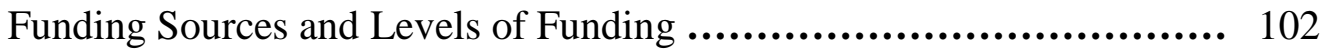

Responses to Open-Ended Question ................................... 105

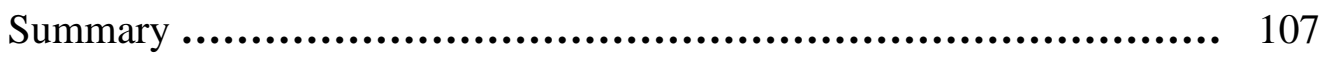

CHAPTER V DISCUSSION.................................................... 109

Research Questions and Overall Model .................................. 109

Effectiveness, Constituencies and Accountability ........................ 121

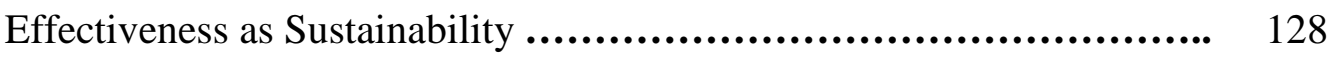

Resource Dependency Theory and Institutional Theory .................. 132 
Study Limitations .................................................... 138

Implications for Practice ............................................... 139

Implications for Theory and Recommendations for Future Research ...... 143

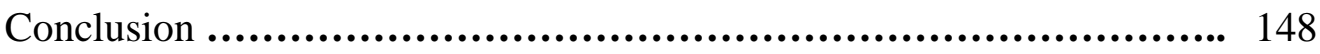

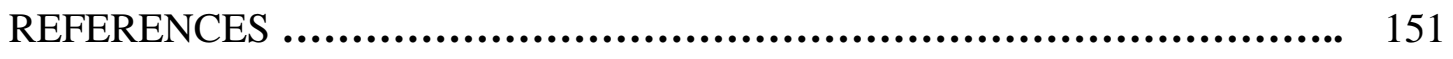

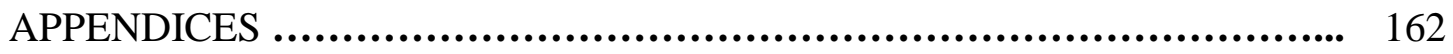

Appendix A: Invitations to participate in the Survey ..................... 162

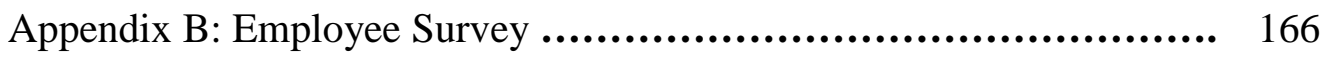

Appendix C: Student Survey …....................................... 178

Appendix D: External Constituencies Survey ............................ 184

Appendix E: Survey Key ............................................... 190

Appendix F: Compilation of Responses to Open-Ended Question ......... 192 


\section{LIST OF TABLES}

TABLE

PAGE

1. Factors Influencing Effectiveness in Public Higher Education Institutions Grouped by Domain .......................................... 55

2. Summary of Operationalized Variables Grouped by Domain .............. 61

3. Guidelines for Effect Size $\ldots \ldots \ldots \ldots \ldots \ldots \ldots \ldots \ldots \ldots \ldots \ldots \ldots \ldots \ldots \ldots \ldots \ldots \ldots \ldots \ldots . \ldots \ldots$

4. Sample Size Guidelines for Six Groups Based on Effect Size ............. 68

5. Study Population and Response Rate by Constituency and by Institution ... 72

6. Dimensions of Effectiveness, Item Loadings and Reliability Values ....... 81

7. Correlations Among Effectiveness Dimensions ........................... 84

8. Analysis of Variance of Effectiveness Dimensions by Constituency Institution 1 ............................................................

9. Analysis of Variance of Effectiveness Dimensions by Constituency Institution 2 ............................................................ 91

10. Analysis of Variance of Effectiveness Domains and Overall Effectiveness by Constituency - Institution 1 .................................... 93

11. Analysis of Variance of Effectiveness Domains and Overall Effectiveness by Constituency - Institution 2 ................................... 94

12. Analysis of Variance for Student Educational Satisfaction Dimension by

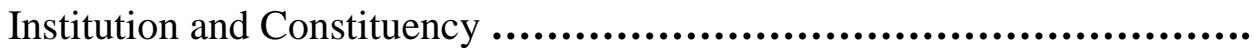

13. Analysis of Variance for Student Academic and Personal Development Dimension by Institution and Constituency ..................................

14. Analysis of Variance for Student Career Development Dimension by

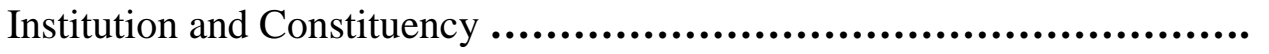

15. Analysis of Variance for Capacity to Maintain or Expand Resources Base Dimension by Institution and Constituency 
16. Analysis of Variance for Faculty, Staff and Administrator Employment Satisfaction Dimension by Institution and Constituency .................... 98

17. Analysis of Variance for Professional Development and Quality of the Faculty Dimension by Institution and Constituency................... .98

18. Analysis of Variance for System Openness and Interaction Dimension by

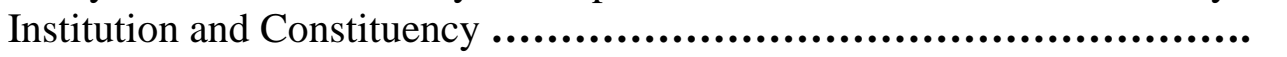

19. Analysis of Variance for Resources for Quality Programs, Faculty and Students Dimension by Institution and Constituency ........................

20. Analysis of Variance for Organizational Culture and Health Dimension by

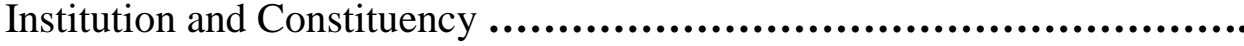

21. Analysis of Variance for Ability to Acquire, Save and Use Resources Effectively Dimension by Institution and Constituency .....................

22. Analysis of Variance for Ability to Overcome Financial Difficulties

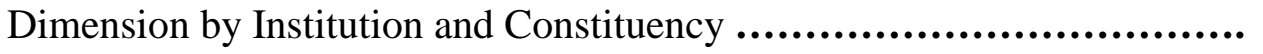

23. Analysis of Variance for Environmental Systems Domain by Institution and Constituency

24. Analysis of Variance for Social Systems Domain by Institution and Constituency

25. Analysis of Variance for Economic Systems Domain by Institution and

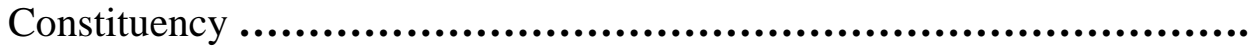

26. Analysis of Variance for Overall Institutional Effectiveness by Institution and Constituency

27. Effectiveness Means by Institution Based on Funding Source and Level ... 104 


\section{LIST OF FIGURES}

$\begin{array}{lll}\text { FIGURE } & \text { PAGE }\end{array}$

1. Visual Representation of Sustainable Development Framework .............. 10

2. Theoretical Model of Effectiveness in Higher Education ...................... 53

3. Revised Theoretical Model of Effectiveness in Higher Education.............. 118 


\section{CHAPTER I}

\section{INTRODUCTION}

The world of today's higher education organizations is characterized by rapid change, economic and political turbulence, and increasing global interdependence. While each of these trends is important by itself, the convergence of these factors and the effects and challenges this convergence presents for colleges and universities is even more important. This turbulent environment places pressure on colleges and universities, forcing them to rapidly change and accommodate many, often competing demands. At the same time, universities are expected to provide graduates who will be able to compete successfully in a global, knowledge-based economy. And, while governments continue their disinvestments in public higher education, they still expect colleges and universities to participate in solving social and economic problems facing today's society (Clark, 1998). The question then becomes whether today's colleges and universities are capable of fulfilling what is asked of them and how can they achieve the balance between being more responsive to the competing demands and preserving their intrinsic values.

\section{Problem Statement}

In order to be more responsive to the demands of their numerous constituencies in this complex, knowledge-oriented, resource-dependent environment, and at the same time preserve their intrinsic values, colleges and universities need to know how effective they are in what they do. This research asked to what degree does institutional effectiveness allow public colleges and universities to operate in a sustained manner over a long period of time while meeting the needs of their constituencies. Effectiveness in this research is 
intended to serve as a proxy for sustainability, thus effectiveness is defined as an "effort to maintain the living triangle" (Cooper \& Vargas, 2004, p. 17) between the social and economic spheres of a university and the environment in which they operate.

Organizational effectiveness in a higher education setting is a complicated notion, difficult to measure. According to Cameron (1978), due to the nature and complexity of educational goals and outcomes, researchers in higher education do not agree on what constitutes effectiveness in higher education. Pascarella and Terenzini (1991) note that many researchers do not consider the traditional measures, such as faculty reputation and research, student body characteristics, etc., as valid measures of institutional effectiveness. Weick (1976), on the other hand asserts that given the uniqueness of higher education institutions, the research on organizational effectiveness in a business setting is not applicable to higher education. Cameron proposes that the problem of measuring effectiveness could be tackled by "identifying a core group of effectiveness criteria that are relevant to organizational members, applicable across subunits, and comparable across institutions" (p. 611).

The purpose of this research was to examine organizational effectiveness and its measurement in higher education environment. In addition, given the changes in how higher education institutions are financed and potential implications for effectiveness, this research explored variations in the effectiveness among public institutions based on the source of public funding they receive and their dependence on these resources. For this research, effectiveness in higher education is defined as creating and maintaining a balance between the economic, social, and environmental factors affecting colleges and 
universities while meeting the needs of their constituencies. Resource dependence in public higher education is defined as a dependence on public funding, which includes state funding, federal funding and public financial aid funding. It has been proposed that the construct of effectiveness is central to sustaining successful operations of higher education institutions. As a result, the following research questions emerged:

1. To what extent is organizational effectiveness defined by environmental, social, and economic factors as they relate to public colleges and universities?

2. To what degree does resource dependence influence the effectiveness of public colleges and universities?

Colleges and universities continue to struggle to meet the demands of multiple constituencies while trying to contain rising costs and increase productivity. This situation has created conditions for increased calls for restructuring universities. As Guskin (1996) notes, "There is a growing public acceptance that colleges and universities are not cost-effective, that our tuitions are too high, and that academic institutions must therefore restructure their operations, much as has happened in other sectors of American society" (p. 26). However, before any attempts for restructuring are made, it is crucial to know where higher education institutions currently stand in terms of their effectiveness. This study intends to provide some answers to this question of effectiveness and move the conversation toward achieving long-term viability and sustainability of these institutions. 


\section{Issue Background}

The complex environment in which higher education institutions operate is partly due to a need to serve and accommodate multiple internal and external constituencies, including faculty, staff, students, parents, alumni, higher education boards, businesses, local governments, legislators, and other elected officials. Often times, these constituencies have competing demands, all of which colleges and universities are expected to accommodate, including demands for an increased access, lower cost, improved quality, and increased effectiveness (Gumport \& Pusser, 1999). Clark (1998) suggests that currently there is an imbalance between environmental demands and institutional capacity to meet them and that this imbalance creates institutional insufficiency. He notes, "Universities require not only an enlarged capacity to respond to changes in the external worlds of government, business, and civic life but also a better honed ability to bring demands under control by greater focus in institutional character. Strongly needed is an overall capacity to respond flexibly and selectively to changes taking place within the knowledge domains of the university world itself" (p. xvi).

Clark's statements imply that colleges and universities are not as effective as they should be in responding to the challenges they face. Yet there is little agreement among higher education researchers and practitioners about what constitutes effectiveness in higher education. This lack of definition and criteria related to effectiveness contributed to the lack of research in the area of organizational effectiveness in higher education. However, if higher education intends to continue its role as a major participant in the 
society, the issue of organizational effectiveness will need to be addressed in a more systematic manner. This study intends to address this issue.

To accomplish this, the study tests the applicability of the sustainability framework as a model of effectiveness in higher education. It suggests modification of the elements of the sustainability and extends the use of the concept of environment as it is defined in the sustainability framework to the concept of environment as defined in organizational theory sense of the term. The use of the sustainability framework with its interlocking circles representing elements of sustainability suggests that institutional effectiveness should be addressed as a system with fully integrated components rather than addressing the individual components of the system separately.

\section{Significance of the Study}

This research seeks to help renew an interest in the work of defining and measuring effectiveness in higher education. It uses the existing literature to explore environmental, social, and economic factors that have an influence on institutional effectiveness. As noted before, the findings from the study are used to consider the applicability of the sustainability framework as a model for assessing effectiveness in higher education. In addition, using a survey to gather information about perceptions of relevant internal and external university constituencies regarding educational outcomes, processes, and environment in higher education, the study investigates differences among constituencies in their ratings of effectiveness. Finally, given the changes in how higher education institutions are financed and the potential implications of these changes for effectiveness, this research explores the degree to which resource dependence, primarily 
dependence on public funding, influences the effectiveness of public colleges and universities.

This research contributes to empirical and theoretical knowledge about effectiveness and the factors influencing it. Its findings can assist higher education institutions to better prepare to respond to challenges and conflicting demands and thus increase institutional capacity. The results will help universities to better understand their constituencies and the expectations these constituencies have of institutions. Furthermore, the study suggests a model of institutional effectiveness by modifying the elements of the sustainability as put forward in the sustainability framework to fit the concepts defined in the organizational theory. Additionally, it offers institutional theory as a basis to explain resource dependence in public higher education. Thus, it is expected that this study will provide important contributions to the field of organizational effectiveness, aid in understanding the role that public funding plays in higher education effectiveness, and contribute to the field of organizational theory more generally.

\section{Summary}

Making informed decisions about institutional actions, strategies and goals is an important feature of effective institutions. This study looks at the factors considered as being influential on institutional effectiveness and proposes a way to measure it. The knowledge about institutional effectiveness positions higher education leaders to build a sustainable future for their institutions. To explore these propositions, the next chapter reviews literature relevant to the concepts put forward in this study. Chapters III and IV describe process used in identifying and developing dimensions of effectiveness, explain 
analyses performed in the study, and present the results of these analyses. Finally, Chapter V discusses the findings of the study and its empirical and theoretical implications. 


\section{CHAPTER II}

\section{LITERATURE REVIEW}

As indicated before, one purpose of this study is to provide more insight into the environmental, social, and economic factors affecting higher education institutions and their relationship to institutional effectiveness. To this end, the reviewed literature addresses previous research on organizational effectiveness with a particular focus on higher education. In addition, the literature on factors affecting higher education is examined to gain a better understanding about the complex internal and external environment in which higher education institutions operate.

To address a need for colleges and universities to respond to pressures of various constituencies, the importance of institutional effectiveness for accountability and improvement purposes is discussed. Finally, given the emphasis in this study on the role of resources in effectiveness, the review looks into the resource dependence through the lenses of resource dependency theory and institutional theory. First, however, given that the proposed research tests the applicability of the sustainability framework as a model of effectiveness, a short history of the sustainability framework development is reviewed, and its utility as a conceptual basis for the current research and the conversion into a model of effectiveness is discussed. Additionally, the definitions of the elements of sustainability in higher education and the rationale for these definitions are outlined. 
Sustainability Framework and Its Application to Effectiveness

One of the research questions in this study seeks to examine the applicability of the sustainability framework as a model of assessing effectiveness in higher education. In order to do that, it is important to first review sustainability framework as it is conceived in its original form and then explain how it is envisioned to be translated into a model of effectiveness. The most often used definition of sustainability is the one put forward in the report Our Common Future by the World Commission on Environment and Development, known as Brundtland Commission, which states that sustainable development is the development that "meets the needs of the present without compromising the ability of future generations to meet their own needs" (as cited in Cooper \& Vargas, 2004, p. 2). This definition captures one of the main findings of Brundtland Commission pointing out that in order to achieve sustainable development, the focus cannot be only on the environment or the economy but "it must be based on the living triangle, a balance between environmental protection, social development, and economic development" (Cooper \& Vargas, 2004, p. 3).

As Cooper and Vargas (2004) note, the efforts to address all three elements of sustainability in a concentrated manner have not been successful thus far and the tendency has been to focus only on one dimension at a time, with environmentalists focusing on environmental protection, economists on economic development and growth, and advocates of social sustainability focusing on social development. These three elements, or pillars, form the basis of sustainability theory as it is known today. The World Conservation Union adopted, in their 2005 report, the use of interlocking circles as 
a model of sustainability (Figure 1) with the intention to "demonstrate that the three objectives need to be better integrated, with action to redress the balance between dimensions of sustainability" (Adams, 2006, p. 2).

\section{Figure 1: Visual Representation of Sustainable Development Framework}

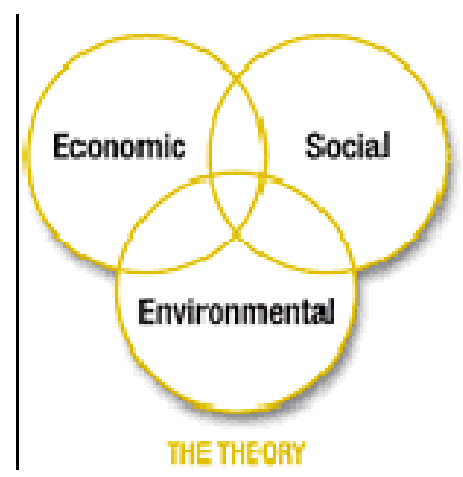

The World Conservation Union Report:http://cmsdata.iucn.org/downloads/iucn_future_of_sustanability.pdf

The use of interlocking circles as a representation of sustainability framework serves as the foundation for the higher education effectiveness framework as put forward in this study. Before moving onto the review of the literature on effectiveness, it is important to consider the meaning and definitions of the three pillars of sustainability in the context of sustainability framework in order to establish its usefulness in examining effectiveness.

According to Esty, Levy, Srebotnjak and de Sherbinin (2005), "Environmental sustainability refers to the long-term maintenance of valued environmental resources in an evolving human context" (p. 11) and it includes among other factors, natural resources, pollution levels, environmental management and general "protection and management of environmental resources and stresses" (Dahl, as cited in Esty, et. al., 2005, p. 12). Economic sustainability refers to a general distribution of the wealth, 
making sure that the "capital accounts are in balance, and investments in wealth generating assets are at least equivalent to their depreciation" (Dahl, as cited in Esty, et.al., 2005, p. 12). Lastly, social sustainability refers to the social aspects of the society and as Dahl notes, "No society can be considered sustainable without attention to the social dimension, including effective governance, social justice and respect for diverse cultural, ethical and spiritual needs" (p. 12).

The three pillars of sustainability require modified definitions in order to fit the framework of organizational theory and to apply them to the higher education setting. First, this study uses the term domain instead of term pillar or dimension of sustainability. Each domain consists of a number of factors forming a conceptual base of the model. The factors within each domain were used to operationalize and measure variables identified as the ones best representing the area of influence for that domain.

While, in general words, organizational environment domain can be defined similarly to how the environmental dimension of sustainability is defined, which is as the "long-term maintenance of valued environmental resources in an evolving human context" (Esty, et. al., 2005, p. 12), it is important to note that the notion of environmental resources in the organizational theory context has a different meaning. It refers to the entities, organizations, groups and individuals an organization interacts with and is affected by them in some way, whether by depending on resources, or as Scott (2003) notes, through the regulative, normative, or cultural-cognitive rules, or in some other way. For the purposes of this research, the environmental domain is comprised of external constituencies, which expect certain outcomes from the institutions and in some 
way can put pressure or otherwise have an influence on public colleges and universities and includes students, state government officials through its legislative education committees, members of the state boards of higher education, local government representatives and employers (businesses and non-profit organizations).

The economic domain of the sustainability framework in higher education refers, in essence, to the ability of an institution to balance its revenues and expenses. More specifically, it refers to the ability of an institution to acquire resources it needs, but also the ability to save and use resources effectively. To paraphrase Dahl (Esty, et. al., 2005), an institution is economically sustainable if its resources are distributed appropriately, its accounts are in balance, and its investments in asset generation are, at least, equivalent to their spending. The social domain of the effectiveness framework, as put forward in this study, is defined as the internal dimension of an institution and refers to the components such as faculty, administrators and staff workplace satisfaction, morale, organizational culture and health of the institution.

As Cooper and Vargas (2004) indicate, one of the primary reasons sustainable development has not been achieved so far is due to the tendency of the groups representing each pillar to clash and advocate their own perspective at the expense of others. It is likely that the same problem exists in higher education. One option then, in the context of organizational theory and as it relates to effectiveness, might be to explore ways to address the domains as a system with fully integrated components rather than addressing the individual components of the system separately. 


\section{Organizational Effectiveness}

Over the years, there has been a considerable disagreement on what constitutes an effective organization. Consequently, researchers have developed numerous theories and models to define and assess organizational effectiveness. Each model, however, has encountered criticism and debate, and the question about the most appropriate model to assess effectiveness remains unanswered. According to Cameron (2005), five models emerged as the best known and most used in the research on organizational effectiveness.

The five models are:

- Goal model

- System resource model

- Internal processes model

- Strategic constituencies model

- Human relations model

The goal model of organizational effectiveness posits that an organization is effective if it accomplishes its goals. According to Georgopoulos and Tannenbaum (1957), organizational effectiveness cannot be studied without consideration of means and ends because "all organizations attempt to achieve certain objectives and develop group products through the manipulation of given animate and inanimate facilities" (p. 535). In addition to the means-ends consideration, Georgopoulos and Tannenbaum recommend organizational flexibility, productivity and strain on its members as necessary criteria in defining effectiveness. Taken together, the authors claim, these four 
factors can potentially be applied to all organizations and therefore be used in assessing their effectiveness.

The goal model encountered criticism from other organizational theorists, mainly proponents of the system resource model (Yuchtman \& Seashore, 1967). Price (1971) responds to the critics of the goal approach, particularly focusing on the issues of goal identification and criteria for evaluation of effectiveness. While acknowledging some problems with the goal approach, such as difficulty with goal identification and lack of general measures to assess effectiveness, Price (1971) contends that the goal approach still offers a better model to address the effectiveness problem than the system resource model does.

For proponents of the system resource model of effectiveness (Seashore and Yuchtman, 1967; Yuchtman \& Seashore, 1967; Pfeffer and Salancik, 2003), an organization is effective if it acquires needed resources. In a critique of the goal model Seashore and Yuchtman (1967) state that, "no single criterion can reasonably be used alone to represent organizational performance" (p. 379) and given the difficulties of identifying the ultimate goal of an organization, the goal approach used in the study of organizational effectiveness appears more "as hindrance rather than as a help" (Yuchtman \& Seashore, 1967, p. 895). Moreover, as the authors note, the relationship between an organization and its environment needs to be incorporated into any framework developed to address organizational effectiveness, and goal approach does not appear to address this relationship (Yuchtman \& Seashore, 1967). Based on this rationale, Seashore and Yuchtman (1967), define organizational effectiveness as organizational 
"ability to exploit its environments in the acquisition of scarce and valued resources to sustain its functioning" (p. 393). Pfeffer and Salancik (2003) add that, "organizational survival and success are not always achieved by making internal adjustments. Dealing with and managing the environment is just as important a component of organizational effectiveness" (p. 4).

Noting problems associated with other models of assessing effectiveness, Steers (1976) suggests that instead of focusing attention on identifying criteria for effectiveness, which he considers an end state, researchers should "view effectiveness in terms of a process" (p. 53-54). In this view, effectiveness is seen largely as the level of success achieved by managers and employees in removing obstacles in organizational goal attainment. Steers (1976) proposes that this multidimensional process model consists of three components: goal optimization, systems perspective, and human behavior, where "the actual criteria for evaluation vary depending on the particular operative goals of the organization" (p. 61). "Because of this", Steers concludes, "it appears appropriate to place greater emphasis on understanding the dynamics associated with effectivenessoriented behavior" (p. 61). It is important to note that Steers viewed this process as contingent and the question of 'fit' with the environment, where as he recommends, managers should "recognize the unique qualities that define their own organization ... and respond in a manner consistent with this uniqueness" (p. 63).

Connolly, Conlon \& Deutsch (1980) offer another perspective in approaching the study of organizational effectiveness. They state, "We argue that an answer to the question "How well is entity X performing?" is inevitably contingent on whom one is 
asking" (p. 212). In other words, they propose a model that allows multiple groups or constituencies to evaluate the performance of an organization with which they are involved based on the criteria identified by these groups. Therefore, strategic constituencies model is a model which defines an organization as effective if it satisfies, at least minimally, its constituencies. According to Tsui (1990), "constituency refers to a group of individuals holding similar preferences or interests pertaining to the activities of a focal organizational unit" (p. 461). Tsui notes how, given that constituencies' judgment is a subjective measure, an organization could influence the perceptions of its constituencies by providing targeted information to the less satisfied groups. This potential for influence, as Tsui notes, could provide a foundation for further study and expansion of the strategic constituencies model.

The last model Cameron (2005) notes as having made an impact in the studies of organizational effectiveness is human relations model. The human relations model states that an organization is effective if its members are satisfied and engaged. Human relations model as espoused by Likert (1967), takes into consideration factors such as motivation, employee satisfaction, decision-making and leadership as influencing organizational effectiveness.

The problem many researchers have with these models is that each leaves something out and is not comprehensive enough to help better understand what constitutes an effective organization. Thus, some researchers (Cameron, 1986b, 2005, Quinn \& Rohrbough, 1981) have attempted to integrate the assumptions that characterize each of the models. The resulting composite model, which they call the competing values 
framework, places effectiveness criteria from each of the five models in four quadrants in opposition to each other. Quinn and Rohrbough (1981) note as advantages of the framework consistency in the level of analysis, integration of perspectives, multiplicity of criteria, relationship among the criteria, an ability to represent the coalitional, dynamic nature of organizations, an ability to allow a comparison across studies, and an explicit definition.

One of the most important features the competing values framework promotes is the paradoxical nature of effectiveness criteria. Weick (1976) and Cameron (1986b), among others, argue that effectiveness is by nature paradoxical. Cameron (1986b) suggests that, "To be effective, an organization must possess attributes that are simultaneously contradictory, even mutually exclusive" (p. 545) and thus, the key to achieving an effective organization is to manage that paradox. The competing values framework, despite its advantages, however, did not solve the effectiveness problem, as the disagreement about the effectiveness criteria remains. Cameron (1982) warns that different models of effectiveness are appropriate for different conditions and the choice depends on a variety of factors. Lewin and Minton also (1986) advocate a contingent approach in the development of effectiveness theory, arguing that one, universal theory would not serve organizations well.

Cameron $(1980,1986 a)$ and Cameron and Whetten $(1983,1996)$ offer seven questions organizations should pose to improve the process of assessing organizational effectiveness. In posing these questions, Cameron (1980) points out that "to gain meaningful results from any organizational evaluation the concept of organizational 
effectiveness must be clearly specified and limited" (p. 79). The questions Cameron and Whetten (1996) consider critical are:

1. "What time frame is being employed? Short-term effects may differ from long-term effects, and different stages in an organization's life cycle may produce different levels of performance.

2. What level of analysis is being used? Effectiveness at different levels of analysis in an organization may be incompatible.

3. From whose perspective is effectiveness being judged? The criteria used by different constituencies to define effectiveness often differ markedly and often follow from unique constituency interests.

4. On what domain of activity is the judgment focused? Achieving high levels of effectiveness in one domain of activity in an organization may mitigate against effectiveness in another domain.

5. What is the purpose for judging effectiveness? Changing the purposes of an evaluation may change the consequences and the criteria being evaluated.

6. What type of data are being used for judgment of effectiveness? Official documents, perceptions of members, participant observations, and symbolic or cultural artifacts all may produce a different conclusion about the effectiveness of an organization.

7. What is the referent against which effectiveness is judged? No universal standard exists against which to evaluate performance, and different standards will produce different conclusions about effectiveness" (p. 275).

Despite difficulties in identifying a proper method of assessing organizational effectiveness, Cameron and Whetten (1996) advocate continued research in this area and offer at least three reasons for it: "First, organizational effectiveness lies at the center of all models and theories of organization. Second, effectiveness was the ultimate dependent variable in organizational research. Third, individuals are constantly faced with the need to make judgments about the effectiveness of organizations" (p. 267). Thus, the authors conclude, "the need to assess organizational performance and to make judgments about excellent practices" (p. 268) remains regardless of whether there is an adequate method to assess it. 


\section{Effectiveness in Higher Education}

If developing effectiveness criteria in business organizations presents a problem, assessing effectiveness in higher education is even more problematic. It has been plagued by a lack of consensus of what criteria should be included in the model. Cameron (1978) notes a lack of clear, measurable goals and outcomes; resistance of academic community to be evaluated in this way because it is seen as potentially restricting academic freedom; prior research, which placed an emphasis on efficiency rather than effectiveness; and the claims that the concept of organizational effectiveness does not apply to colleges and universities because they are, so called, loosely coupled systems. To address these critiques, Cameron focused on identifying organizational characteristics, rather than goals. To that end, he developed nine criteria intended to measure effectiveness of higher education institutions focusing on the organizational level to allow for comparison among institutions.

A major criticism of higher education effectiveness research is that current theories do not capture the characteristics of a majority of institutions of higher education. In addition, there is little agreement among higher education researchers and practitioners on what constitutes effectiveness in higher education. Webster (1981), for example, attempted to identify the most common methods of evaluating effectiveness in higher education and came up with the following: "reputational ratings" by peers or experts, citation counts of faculty members' work, faculty awards and honors, student achievements after graduation, scores of entering students on national exams, and institutional resources. Cameron (1986a), on the other hand, contends that, "In each case, 
major flaws are associated with each of these criteria, the most important of which is that they apply only to 50 or so of the best known institutions in the country" (p. 89). Cameron goes on to say, "Unfortunately, the group of institutions for which these six criteria don't apply compose over 95 percent of the colleges and universities in America. Aside from the few schools with high visibility and traditions of academic excellence, most institutions of higher education are left without obvious criteria to assess their organizational effectiveness" (p. 89).

Indeed, as Cameron (1986a) points out, those institutions that may emphasize meeting local needs, do not have visible athletic programs, or focus more on teaching than research and publications, are left out when effectiveness is measured in traditional terms. Similarly, institutions that emphasize student learning over other traditional criteria are often excluded from this discussion. Schmitz (1993), who undertook a study of the validity of some criteria used by U.S. News \& World Report to assess effectiveness in higher education, such as student ability, instructional budget per student, faculty characteristics, etc., supports these contentions. Schmitz's sample used modified Carnegie classification and included national universities, national liberal arts colleges, as well as regional institutions. More specifically, Carnegie classification was modified by grouping institutions based on the region of the country where they are located and four out of the ten categories were used in the study: national universities and colleges, national liberal arts colleges, Midwestern (regional) liberal arts colleges, and northern (regional) colleges and universities. 
Schmitz's major finding is that indicators studied were valid for national but not valid for regional schools. He used a total of eight indicators divided into input (acceptance rate, mean entrance test score, class standing), process (faculty/student ration, faculty background, instructional budget) and outcome (retention, graduation) indicators. He points out that some of the criteria are not appropriate in determining whether an institution is effective and goes on to say, "The findings assert a more difficult problem with the definition of quality and irrelevance of selectivity and resource indicators for institutions that are nonselective, public (resource-limited) and diverse in mission" (p. 517). Consequently, this lack of criteria and the inability to include broad spectrum of colleges and universities, as noted previously, contributed to the lack of research in the area of organizational effectiveness in higher education. Nonetheless, if higher education intends to continue its role as a major participant in the society, the issue of organizational effectiveness will need to be addressed in a more systematic manner.

While, as noted above, organizational effectiveness in higher education is an understudied concept, Cameron's contribution to the field is significant. In addition to the work he did either by himself or in collaboration with others to test and refine his model, several other researchers have used his model to assess effectiveness in higher education. Clott (1995) studied the relationship between Cameron's dimensions of effectiveness and predictor variables of managerial strategy, organizational culture, change in the external environment and resource dependency in the schools of business in the United States and Canada. His findings show the existence of the relationship for each predictor, but the strength of this relationship is not the same among the predictors. Organizational culture 
has been shown in his study to have the most important influence on organizational effectiveness, followed by limited support for managerial strategy as indicator of effectiveness.

Smart and St. John (1996) explored the relationship between organizational culture and Cameron's dimensions of effectiveness with a particular focus on culture type and culture strength of studied colleges and universities. Cameron and Ettington (1988) proposed a typology of organizational cultures consistent with the competing values framework of organizational effectiveness mentioned earlier. Additionally, they found that Jungian framework of cognitive types, which looks at the ways individuals gather and evaluate information, "lie at the heart" (p. 371) of competing values framework. Based on this, they developed four culture types (clan, adhocracy, hierarchy, market) noting that each type has opposite characteristics with one of the types and shares some characteristics with other two remaining types. The strength of the culture concept stems from the growing agreement among researchers such as Saffold, Peters and Waterman, Deal and Kennedy, and others (as cited in Smart \& St. John, 1996) that the contribution of the organizational culture to performance depends on that culture strength. Strong cultures are defined as "congruence between espoused beliefs and actual practices, whereas weak cultures are characterized by incongruence between espoused beliefs and actual practices" (p. 223).

Smart and St. John (1996) found that culture type has a stronger effect on organizational effectiveness than culture strength, but the differences are more stated on campuses with strong than on campuses with weak cultures. Smart and Hamm (1993b) 
investigated the utility of Cameron's construct in two-year colleges. Besides testing the applicability of Cameron's nine dimensions of effectiveness, they also investigated the perceptions of organizational effectiveness among two-year colleges with different missions. The results support the applicability of nine dimensions in two-year colleges and show that the effectiveness of these institutions varies significantly based on their mission. In addition, to this study, Smart and Hamm (1993a) undertook another study in which they explored the extent to which a sample of two-year colleges differed in the effectiveness based on the dominant type of their organizational culture. They found wide differences in the effectiveness of two-year colleges depending on whether their dominant culture is clan, adhocracy, hierarchy, or market with the adhocracy culture appearing to be the most effective.

Cameron's construct of effectiveness has been tested internationally as well. Lysons (1990), and Lysons and Hatherly (1992) have tested this construct in Australian and United Kingdom context respectively. While findings in Australian context support only four of Cameron's nine dimensions, findings in the U.K. setting show much higher level of reliability for the nine dimensions studied. According to the authors, a possible explanation for differences in results lies in cultural differences between the countries and the link to reputation and resources which exist in both U.S. and U.K. but is lacking in Australian higher education.

As can be seen from the review of the literature, defining effectiveness and developing criteria to assess it presents a problem in both business organizations, as well as in higher education organizations. As the literature review revealed, a major criticism 
of the higher education effectiveness research is that current theories do not capture a majority of institutions of higher education. To address this problem, this research makes an assumption that all colleges and universities have some combination of environmental, social, and economic variables influencing their operations. While public and private colleges will differ to some degree in what constitutes each of these groups, the three domains nevertheless provide a way to define effectiveness criteria in a systematic manner.

While the construct of effectiveness and the criteria defining it is an underdeveloped area of study, the relationship between the factors influencing higher education institutions and effectiveness is even less explored. Some studies have addressed a few of these factors, but a comprehensive study of this problem has not been undertaken. This research attempts to address this gap. To that end, the next section of the literature review explores the environmental, social, and economic factors as they relate to higher education institutions and their effectiveness with a particular focus on public four-year institutions.

\section{Universities as Environmental Systems}

Higher education institutions do not exist in a vacuum; they are embedded into their environment and often influenced by it. Consequently, this environment (public, state government, local government, businesses, students and parents) often has an impact on decision-making and strategy in colleges and universities. If the fiscal constraints higher education faces are added to this list, then it is not surprising that colleges and universities are forced to reconsider how they do their work and review their 
costs, productivity and effectiveness. This dependence and impact of the external environment has not always been as prominent in higher education as it has become in recent times. Roherty (1997) notes, "In a clinical sense, an exogenous variable may be thought of as an agent introduced from, or produced, outside of the organism. Higher education has a long history of ignoring exogenous agents, preferring instead to define the academy from within. This tendency, traditionally defended under the rubrics of academic freedom and faculty governance, served it well over many centuries and well into the twentieth century. Now, however, these outside agents appear eminent, and their clamor may well shape the future of higher education" (p. 13).

The environmental factors influencing higher education bring to the forefront the discussion about the role of public higher education in a society. As Gumport (2000) states, there are two conflicting views about the role of public higher education in a society. One view is of higher education as an industry, with activities and priorities similar to the ones businesses espouse: "to produce and sell goods and services, train some of the workforce, advance economic development, and perform research" (p. 7071). All of this should be accompanied by flexibility and ability to respond to changes in a rapid manner, adjust programs and activities as needed, and improve efficiency and customer satisfaction. On the other hand, for those who view higher education as a social institution, its primary functions should include "cultivation of citizenship, the preservation of cultural heritage(s), and the formation of individual character and habits of mind" (p. 71). 
The conflicting views about the role of higher education are reflected in how public higher education is financed. Roherty (1997) states that, "state government has in many cases adopted a policy of passive resistance in financing higher education" (p. 21), where governors and legislators "have allowed natural forces to take their course" (p. 21). This withdrawal of state support has led colleges and universities to supplement their revenues with increased tuition, intensify the pursuit of research funding and private giving, as well as to redraw the lines between universities, state and market.

Slaughter and Rhoades (2004) argue that these lines cannot be clearly distinguished any more and offer a theory of academic capitalism to support their claim: "Theory of academic capitalism explains the processes by which colleges and universities are integrating with the new economy, shifting from a public good knowledge/learning to an academic capitalist knowledge/learning regime" (p. 7). They go on to explain: "The theory of academic capitalism focuses on networks...that link institutions as well as faculty, administrators, academic professionals and students to the new economy...Together these mechanisms and behaviors constitute an academic capitalist knowledge/learning regime" (p. 15). Slaughter and Rhoades point to the connection between the changing resource mix and the academic capitalist knowledge/learning regime. In addition, given that students and their parents pay an increased share of the tuition, they are also becoming more conscious about what they expect to receive from colleges and universities in return. Thus, "these changed expectations reshape student identity from that of learner to that of consumer" (p. 12). 
Colleges and universities, as part of the environmental system are becoming more responsive to environmental constraints, but Gumport (2000) warns that this responsiveness is "both impressive and troubling. It is impressive because such adaptive responses just may help public higher education survive... and troubling because of the potential damage to public higher education as an intellectual enterprise" (p. 69). Understanding universities as systems means understanding how parts of a university interact with each other and how they fit into the larger system. As Katz and Kahn (2005) assert: "System theory is basically concerned with problems of relationships, of structure, and of interdependence" (p. 482) and "living systems, whether biological organisms or social organizations, are acutely dependent upon their external environment and so must be conceived as open systems" (p. 482-483).

Universities are considered open systems, and even though they have boundaries, these boundaries are permeable and enable interactions with the environment (Birnbaum, 1988). According to Simsek and Louis (1994), "By viewing organizations as adaptive organisms that strive toward equilibrium under changing environmental conditions, systems theory shifted the focus of organizational research from exclusive attention to internal conditions to a concern with the relationship between the organization and its environment" (p. 670). It is important to note that organizations differ in permeability of their boundaries, which can create different responses to the environment. In addition, the degree of the complexity and uncertainty of environmental demands can contribute to a variation in institutional response (Birnbaum, 1988). 


\section{Universities as Social Systems}

Universities are social systems, which are both shaped and shape the lives of individuals associated with them. Thus, it is important to examine the influence of employees and organizational culture, as internal organizational components, on universities and their effectiveness. Even though external forces represent an important and powerful factor influencing institutions of higher education, it can be argued that internal forces can and sometimes have even bigger impact on shaping the character of these institutions. Tierney (1988) contends that these internal forces originate from values and goals of the people working in a university and goes on to say, “An organization's culture is reflected in what is done, how it is done, and who is involved in doing it. It concerns decisions, actions, and communication both on an instrumental and a symbolic level" (p. 3). Thus, it is important to consider the role of culture in achieving organizational effectiveness.

Morgan (1997) explains that culture as a set of "patterns of belief or shared meaning, fragmented or integrated, and supported by various operating norms and rituals can exert a decisive influence on the overall ability of the organization to deal with the challenges that it faces" (p. 129). The results of some studies (Clott, 1995; Welsh \& Nunez, 2005) suggest that organizational culture has one of the most, if not the most important influence on organizational effectiveness in higher education

These findings are complicated by Bergquist's (2008) claim that there is not only one, but rather six cultures simultaneously existing in any given college or university. They are: collegial, managerial, developmental, advocacy, virtual and tangible culture. 
The interplay of these cultures creates a special dynamic as each member of the university community participates in one or more of these cultures and interacts with others from the same or different culture. Understanding these six cultures and interactions that occur among them can help higher education leaders to improve the operations and effectiveness of colleges and universities. Bergquist (2008) notes that a desire to understand cultures is reasonable, given that it helps us to sort out complex organizational dynamics and create order out of them.

Bergquist (2008) points out that it is not possible in most instances to create a unified culture in the academy, even though some authors such as Tierney, advocate for this. Bergquist agrees that having a unified culture in an organization is a great asset, but warns against creating a unified culture dominated by one of them without considering benefits other cultures can provide. As indicated before, Smart and Hamm (1993a) showed evidence that the type of culture has an impact on effectiveness. While the typology they used differs from Bergquist's, their findings do have implications for how institutional leaders approach the subject of organizational culture as it relates to effectiveness.

The challenging environment and the changes higher education institutions, especially public ones, are undergoing have been noted as one of the defining moments in higher education in recent history (Clark, 1998). The changes in the structure of universities and the nature of work for faculty, staff and administrators are some of the changes impacting internal functions in higher education. Gumport (2000) discusses academic re-stratification, which is "based upon the increased use-value of particular 
knowledge in the wider society and exchange-value in certain markets" (p. 81). Some of the consequences of re-stratification include engaging in commercial activities, such as patents and licenses, and copyrights. This in turn contributes to the "commodification" of academic functions, despite the best intentions of faculty and administrators to improve colleges and universities.

One of the issues associated with the question of improvement is, as Slaughter and Rhoades (2004) point out, that faculty and administrators often do not have the same view of what constitutes an improvement: "Senior level administrators seek to enhance the annual operating budgets of institutions. Managerial professionals are concerned with expanding and making permanent their professional positions and services", while "Faculty often try to maintain their control of the curricula and expand their share of profits from external revenues derived from intellectual property" (pp. 157-158). While generalization of these claims can be questionable, the reality is that different internal constituencies often have different goals and aspirations as well as demands placed on them. And the demands are continuously increasing as the university functions become more complex, contributing to the divisions in the university community.

These changes lead to a sharper division and increased tension between faculty and administrators, since, "external calls for greater accountability and demonstrable outcomes, institutional pressure for faculty to generate revenue, and the necessity of keeping up with the never-ending expansion of new knowledge all conspire to create seemingly endless demands and expectations of faculty members" (Gappa et al., as cited in Bergquist, 2008, p. 70). In addition, due to resource constraints, universities are 
increasingly relying on part-time faculty, substituting them for tenure and tenure-track faculty, which while lowering the costs, changes the composition of the faculty as a whole and has implications for processes such as faculty governance and control over curriculum. On the other hand, as Brigham (1996) states, administrators "are told to transform undergraduate education, reshape faculty roles, practice quality, serve new constituents, work in teams, and honor diversity - all entailing deep changes of mind and habit that no committee, no matter how powerful, can simply order up" (p. 28).

\section{Universities as Economic Systems}

Balancing revenues and expenses has become a constant struggle for public colleges and universities. Previously major source of revenue, state appropriations occupy a smaller and smaller portion of total revenues for public colleges and universities. To fill the gap, institutions are turning to other sources: increased tuition, auxiliary revenues, increased research funding, gifts, endowments, public/private partnerships, and partnerships with local governments. Each of these sources brings with it a host of issues for consideration. One of the reasons state appropriations have been steadily declining over time is the public perception that a college degree benefits individuals more than society, and therefore individuals should pay a greater share for their education.

Fairweather and Hodges (2006) examine the benefits, both private and public of a college degree, pointing out that private benefits are the ones that accumulate to an individual and to a family, while public benefits provide value to a community, state, nation, and beyond. The authors suggest that costs and benefits of higher education can 
be considered by looking at the return on investment in both public and private area. They argue that the "return on investment is crucial to individuals and to state governments because it estimates whether an additional dollar invested in higher education achieves the desired benefits in comparison to other types of investment" (p. 4). They note that private and public benefits can be divided between the social and the economic.

Private social benefits include students' academic, personal, and skill development; these benefits are long lasting. More specifically, college graduates report that they are satisfied and enjoy life, have improved health and life expectancy, exhibit better consumer decision making, and are better prepared for a successful career. Some of the private economic benefits include higher earnings and benefits, higher savings rates, and lower rate of unemployment.

As public social benefits, Fairweather and Hodges (2006) list "advancement of knowledge, preservation of culture, support for and enjoyment of the arts and culture, discovery and encouragement of talent, attitudes toward lifelong learning, and the advancement of social welfare" (p. 8). In addition, college graduates contribute to the reduced crime rates, increased charitable giving and community service, as well as to an increased quality of civic life. Moreover, living in the close proximity to an institution of higher education provides benefits to residents by enabling them to, for example, use university facilities for various purposes or participate in educational, cultural or recreational activities organized by the institution. 
Finally, public economic benefits to which higher education contributes are increased tax revenues from college graduates but also from university employees. In addition, college graduates are less likely to receive public assistance, putting less pressure on public welfare system, and are more likely to be in a better health thereby reducing costs of health care. Institutions of higher education contribute significantly to the economic growth of the region they are located in by spending in the local community as well as by generating jobs beyond their campuses. All this points to the conclusion that, "return to the state from investing in public higher education always exceeds the original investment, sometimes dramatically so" (Fairweather \& Hodges, 2006, p. 10).

This examination of public and private benefits brings up again the questions concerning the role of higher education in a society. As Gumport (2000) states, "On the one hand, there is a call to protect: How can higher education protect its legacy, including decades of public investment in an enterprise whose strengths must not be diluted or deteriorated for short-term market demands? On the other hand, there is a call to respond: How can higher education redefine itself to attend to the signals of those it is supposed to serve?" (p. 88). Moreover, structural changes related to financing of higher education institutions are of no less importance, the most notable being a shift from state funding to tuition as a major source of revenue.

State Appropriations vs. Tuition as Revenue Source There are over 4,200 degree-granting, public and private institutions of higher education in the United States (Ehrenberg, 2007). Even though the majority of these 
institutions are private, about two thirds of all undergraduate students are enrolled in public four-year institutions. The disparity in the average tuition and fees students pay at public and private institutions is about four to one (The College Board, 2007), but neither price reflects the full cost of education for institutions.

According to Winston (1999), undergraduate college education is subsidized at both, private and public institutions, but at public institutions, those subsidies come from state appropriations in addition to other sources, such as endowments, alumni and other donors. Despite subsidies, last three decades have seen a dramatic increase in tuition and fees at public and private colleges. Ehrenberg (2007) notes that the rates of tuition increase at four-year public institutions have been the same or even greater than at private colleges. However, as Ehrenberg (2007) adds, "While tuition increases at privates have always been associated with increases in expenditures per student, tuition increases at publics have often been associated with decreases in expenditures per student" (p. 11). One of the main reasons for this is a steady decrease in state funding of public higher education institutions, forcing institutions to charge higher tuition and fees and in this way make up for lost revenues.

According to Roherty (1997), state governments have four core businesses funded with general fund: K-12 education, health care, higher education, and public assistance. The problem with this funding is that increased costs put pressure on the ability of states to fund their core businesses, with higher education being the only one viewed as a discretionary item in the budget and therefore suitable for budget cuts. Additionally, it is one of the few that has alternative sources of revenue, to which institutions can tap in to 
substitute for state funding. Faced with, what Ehrenberg (2006a) calls perfect storm, public colleges and universities are finding themselves looking for other ways to pay for expenses, one of the primary being tuition increases. A shift from public support to reliance on tuition revenues indicates privatization of public higher education leading some university presidents to claim, "Once we were state supported. Then we were state assisted. Now we are state located" (as cited in Thelin, 2004, p. 36).

One of the consequences of the view that higher education benefits an individual more than a society, and is a private rather than a public good, has been shifting the cost of higher education from public to individual. Breneman and Finney (1997) warn that this shift has important implications which need to be taken into consideration and note, "As states go about the task of determining their priorities and as public institutions begin to adopt the revenue-raising strategies of private institutions, it is crucial that policy makers consider the effects of a general shift from state to private support of public higher education" (pp. 52-53).

One of these effects is the effect of tuition increases on students especially as it relates to affordability, educational opportunity, and access. The transfer of financial responsibility to students forces them to increase their dependence on grants and loans in order to pay for college education. Furthermore, as a result of the political pressure from voters, state and federal government have begun to change the nature of financial assistance to students. Middle class has disproportionately benefited from these changes because assistance has increasingly moved away from need-based aid. The federal government responded to pressures by increasing support for loans and tax credits as 
forms of financial aid, not by increasing Pell Grant awards. States, on the other hand, have started to shift more resources away from direct institutional support to grant aid given directly to students, but that aid is increasingly merit based (Ehrenberg, 2006a).

This movement from educational opportunity to middle-class support has a negative effect on educationally disadvantaged students, especially since as Ehrenberg notes, "high school graduates who qualify for these awards are disproportionately white and middle- or upper-income. Hence, the growth of these programs can be understood primarily as a response to large voting blocs concerned about rising college tuitions, not as an effort to increase access for underrepresented groups" (p. 49).

Faced with this situation, public higher education institutions have an option to provide grant aid themselves to maintain an access for lower income students. This option however is often in competition with other institutional priorities, especially given the increased national focus on rankings and associated indicators of quality. Hossler (2004) points out that this places public institutions with a relatively open-access mission at a disadvantage, since a significant part of these rankings includes high school grade point average, standardized test scores, and high school class ranks. Thus, institutions have to use limited revenues to balance often-competing goals of access, diversity, and quality. Hossler reminds us that, "Unfortunately, as a nation we have not yet achieved the societal equity goals we espouse; and because a disproportionate number of minority students of color are from low-income families, a disproportionate number of students of color may be less likely to score well on traditional indicators of academic quality" (p. 159). 
The combination of state cuts, tuition increases, and admissions restrictions has made many public institutions less accessible and less dedicated to expanding educational opportunity for the broader population (Alexander, 2006). This has implications for public policy, since "many argue that the increased essentiality of higher education to individuals' life chances in modern society makes it all the more necessary that public policy ensure equity of access across an increasingly diverse population" (Zumeta, 2004, p. 81). A challenge for public colleges and universities is to find a way "to sustain a system of higher education that is characterized both by quality and broad accessibility" (Zemsky \& Wegner, 1997, p.66), because as Zumeta (2004) notes, "Higher education faces the odd paradox of being simultaneously highly sought after by key societal elements, and sharply constrained in its ability to gain effective political support and thereby adequate financial sustenance - at least from its traditional sources - to realize its own aspirations and those society holds for it" (p. 79)/.

\section{Knowledge Economy as Revenue Source}

In addition to raising tuition, public colleges and universities have started to pay more attention to other sources of revenue further blurring the line between public and private. According to Slaughter and Leslie (1997), activities such as some forms of research grants and contracts, technology transfer, public-private partnerships, partnerships with various levels of government, service contracts, and others are beginning to change the nature of work for faculty and administrators. Increasingly, public higher education is forced to look for external funds to fill a hole left by inadequate state funding. To secure these funds, faculty and administrators are using 
market-like activities to a greater extent than before. Slaughter and Leslie (1997), and Slaughter and Rhoades (2004) call these efforts academic capitalism, a process of integrating universities with the knowledge economy, which essentially shifts the focus away from public good knowledge.

Slaughter and Rhoades (2004) point out that this new economy is not a cause for the rise of academic capitalism, but, at the same time, it is not easy to separate knowledge and the new economy. Since universities are places of knowledge creation and "the new economy treats advanced knowledge as raw material that can be claimed through legal devices, owned, and marketed as products or services" (p. 15), universities, in their quest for new revenues, are deciding to become participants in the new economy, changing in the process the academia. Slaughter and Rhoades do not see faculty and administrators as actors being "corporatized" (p. 12). Rather, in the climate where the search for new external sources of revenue is of ever increasing importance, they themselves initiate activities related to academic capitalism. Indeed, as the authors note, the continuing decrease in government funding has played a role in making academic capitalism legitimate. Therefore, even in occasional periods of increased state revenues for higher education, the activities pursuing market strategies still increased as well.

\section{Costs in Higher Education}

In addition to a need to keep tuition at a reasonable level and to enhance revenues, colleges and universities are also under pressure to keep their costs as low as possible. While operational costs keep rising due to increased regulations and costs related to accountability reporting, technology costs, competition between institutions, operational 
inefficiencies, budgeting systems, administrative lattice, academic ratchet, employee health and retirement benefits, and others, public and state governments are still asking colleges and universities to contain and reduce costs of their operations.

Massy (1996) asserts that there is a tension between being responsive to the external demands and being true to one's intrinsic values and that tension should be addressed. In Massy's view, this tension can be addressed by effective resource allocation process, which is based on the system of incentives guiding institution's spending. This system "will allow institution to achieve a proper balance between its intrinsic values and those of the marketplace. Institutions that ignore the market-place risk financial dislocation; those that ignore intrinsic values - as bound up in the academic mission and vision, for example - will come to behave like an ordinary business enterprise" (pp. 4-5). However, as Massy (1996) notes, there is no one intrinsic value within any given institution of higher education. On the contrary, there is value diversity in an institution depending on the academic discipline, educational purpose and self-interest. These different values and the interests they promote create a situation where resources are diverted from institutional goals. Massy notes that one of the keys for effective resource allocation is recognizing and managing this value diversity and self-interests that exist within an institution as well as an ability to manage complexity.

The traditional approach in dealing with the problems of value diversity, selfinterest, managing complexity, and balancing values and market forces is to have a central control over resources. It has been recognized, however, that the traditional budgeting systems are not well positioned to adequately address issues which confront 
higher education. In particular, it has been noted that the failure to understand changes in the society and the demands to address these changes marginalizes colleges and universities and even endangers their existence (Massy, 1996). At the same time, any change deviating from the traditional resource allocation model will be confronted with resistance based on the assumptions ingrained in the culture of colleges and universities. According to Massy (1996), the solution lies in finding a way to "decentralize budgetmaking authority without abandoning institution-level values and priorities" (p. 5). In making a case for a resource allocation reform, Massy (1996) asserts, "Absent decentralization, rigidities and misallocations will build up to the point where the institution cannot remain true to its mission or respond effectively to environmental threats and opportunities" (p. 10).

Two processes often associated with increased costs in higher education are an administrative lattice and academic ratchet. According to Massy (1996), the administrative lattice denotes "the proliferation and entrenchment of administrative staff at American colleges and universities. The term lattice refers not only to this increase in staff but also to its effects on an institution's operations and costs." (p. 80). And while the effect of lattice is relatively visible, the changes caused by academic ratchet are more difficult to detect. Ratchet refers to "irreversible shift of faculty allegiance away from the goals of a given institution, toward those of an academic specialty...The increasing outputs or primary gainers from the ratchet are research, publications, professional services (consulting), and curriculum specialization. Diminishing outputs or the primary 
losers include teaching quality, advising, mentoring, tutoring, and curriculum structure" (Massy, 1996, p. 81).

Whether we are talking about additional revenue streams or efforts to decrease costs, it is important to consider these efforts in light of the shift from public to private support of public higher education. Breneman and Finney (1997) suggest that given this shift, a better understanding of the effects of an increased reliance on tuition and other private revenues sources is required. For example, what effects will the change in how institutions are financed have on public policy goals such as access, economic development, student financial aid, educational opportunity, and others. As Zemsky and Wegner (1997) pose, "Necessarily, then, the basic questions concerning the financing of higher education - "Who Pays? Who benefits? Who should pay?" - will need to be reasked, in some cases as a means of distinguishing between the different missions that higher education fulfills: "What should be subsidized? From what sources? For what purpose?"”' (p. 67).

\section{Effectiveness and Accountability}

Over the last several decades, as higher education costs have risen and budgetary constraints put pressures on the ability of states to fund core areas of operation including higher education, questions have increasingly been asked about how colleges and universities do their work and how they spend their money. In the 1970s, responding to concerns about quality and return on investment, state policymakers have started monitoring the performance of public colleges and universities (Layzell, 1999). To that end, legislative and regulative bodies have used a variety of models to connect quality 
and accountability. As Nedwek (1996) notes, there is a "steady movement away from quality assurance through internal mechanisms of control toward externally driven description of activity monitored by government agencies and other organizations" (p. 47).

Over time, this interest in higher education institutions and the quality of their work expanded from legislative and regulative bodies to tax-payers, students, parents, businesses, employers, and funding bodies other than government. Thus, a need to assess institutional performance and effectiveness to demonstrate accountability to the public has taken new importance for colleges and universities, especially public ones. In addition, of no less importance is assessment for improvement, which serves internal purposes of an institution. Inevitably, a need to do assessment serving two different purposes created a tension, especially given that as literature review revealed there is no agreement on what constitutes effectiveness in higher education.

Frye (1999) makes a distinction between assessment and accountability, but it seems appropriate to modify these terms to assessment for improvement and assessment for accountability to account for terminology developed in this area in the last decade. He defines assessment for improvement as "a set of initiatives we take to monitor the results of our actions and improve ourselves" (p.1), while assessment for accountability is seen as "a set of initiatives others take to monitor the results of our actions, and to penalize or reward us based on the outcomes" (p. 1).

Ewell (2009) notes changes in the assessment movement in the last couple of decades and points to a changed policy environment, which brought higher education to 
the center of attention. This new "policy centrality" (p. 6) of higher education is promoted at the state and federal level and supported by the business communities and it refers to the need to raise educational attainment of U.S. citizens and help improve the country's competitive position in the world. Ewell goes on to say that transparency and student learning outcomes are central to this changed accountability environment. Thus, states have embraced this "public agenda" (p. 10) for higher education where, according to Ewell, focusing on learning outcomes "meant focusing attention less on how individual institutions perform with respect to learning and more on determining particular strengths and deficiencies in the "educational capital" of the state as a whole" (p. 10).

Huisman and Currie (2004) note that this new focus denotes a shift from professional to political accountability. According to Romzek (2000), there are four basic types of accountability encountered in the public sector, hierarchical, legal, professional, and political, with the last two found most often in higher education. Professional accountability allows a high degree of autonomy to an organization or individuals in their everyday work. It is usually used when a specialized knowledge and skills are required to perform a job and thus the decisions about performance are deferred to professionals with recognized knowledge and expertise. Political accountability, on the other hand, refers to the responsiveness of administrators to key stakeholders, including government officials, funders, general public and others.

Romzek points out that the biggest difference between the two types of accountability is the source of the standard for performance. For professional type 
accountability, the standard is the "individual's own internalized standards and judgment" (p. 26), while the source of the performance standard in the political accountability is "the other and whether the administrator had successfully responded to the others' concerns" (26). This shift is also reflected in how assessment is viewed from assessment as an internal activity focused on improvement to externally oriented assessment for accountability. And as Ewell (2009) states, this shift is not of small importance because "Adopting either of these two perspectives affects institutional choices about what and how to assess, how to organize assessment tasks and strategies, and how to communicate assessment results" (p. 3).

A need to conduct assessment for accountability in order to respond to the numerous external constituencies, does not, however, obviate a need to conduct assessment for internal purposes including for improvement. And this conflicting purpose is what, according to some (Huisman \& Currie, 2004; Ewell, 2009), creates a tension in higher education. Huisman and Currie (2004) conducted a study about the effect of accountability on higher education and found that from the internal perspective, it is generally not viewed as contributing positively to the quality of education. Nevertheless, the authors found indications that the shift from professional to political accountability has already occurred and universities are left to manage this shift as best as they can. Ewell (2009) warns that managing this tension is crucial because "Giving too much attention to accountability risks losing faculty engagement - effectively suppressing the sustained, critical self-examination that continuous improvement demands. Devoting attention solely on the internal conversations needed for improvement, on the other hand, 
invites external actors to invent accountability measures that are inappropriate, unhelpful, or misleading" (p. 20).

Thus far, public, legislators, governors, and accrediting agencies have dominated the discussion and decision-making about effectiveness and accountability in higher education. In many cases, funding decisions are made based on the results of these discussions, leaving colleges and universities to comply or face financial implications. Perhaps, it is time for higher education to take a lead in these conversations. While a number of national associations are already addressing the issues of accountability, effectiveness and educational quality, there is much more to do. This will require colleges and universities to rethink how they do their work and how they define performance. It will also require a broader engagement within the university community. The challenge is developing criteria that convey what is important to both internal and external constituencies, as well as allows clearer understanding about what constitutes an effective organization. The current study aims to move a step further in finding solutions to some of these issues.

\section{Effectiveness and Resource Dependence}

One purpose of this study is to explore the role of resource dependence in effectiveness of public higher education institutions. The resource dependence is linked to the external environment within which universities operate, and affects the way they interact with that environment. Organizations are systems and elements of this system affect and are affected by each other and cannot be viewed in isolation (Scott, 2003). This brings up, as Scott and Meyer (1996) note, "the idea that organizations are deeply and 
essentially embedded in wider institutional environments" as opposed to "conceptions of organizations as bounded, relatively autonomous, rational actors (p. 1). In order to be successful, organizations have to learn how to manage this environment.

The notion that organizations depend on other organizations for resources introduces special dynamics in their relationships. As Pfeffer and Salancik (2003) point out, dependence helps us understand the influence of the external environment on organizational decision making and indicates the power external organizations have in a given organization. Dependency is really "a measure of how much these organizations must be taken into account and also how likely it is that they will be perceived as important and considered in the organizations decision making" (p. 52).

According to Pfeffer and Salancik (2003), the power of resource dependence will depend on the number of funding sources an organization depends on. The rationale here is that if resources are controlled by a small number of organizations, the affected organization will be more dependent on them due to a limited pool of resources available and thus these organizations will have more power over it. Resource dependency theory as developed by Pfeffer and Salancik in the 1970s puts the dependence on resources at the center of explaining organizational behavior, stating that organizations conform to the expectations of their funders.

While important, resource dependency theory does not fully explain the dependence on resources colleges and universities, especially public ones, experience. It can be argued that other institutional factors, such as political, legal or social environment, can and many times do have a greater role in explaining the way 
dependence affects universities. Thus, institutional theory, which states that, "institutions are multifaceted systems incorporating symbolic systems - cognitive constructions and normative rules - and regulative processes carried out through and shaping social behavior (Scott, 1995, p. 33) could perhaps better explain the dependence higher education institutions face including dependence on resources and the way they approach this dependence.

Scott (1995) identifies three elements or pillars of institutions that are used as a means to influence organizations with which they interact. Scott (1995) argues that "institutions constrain and regularize behavior (p. 35) and Andrews (2008) points out that institutions such as governments can significantly affect an individual organization in areas such as strategic leadership or resource allocation. Andrews (2008), who studied the interaction and the presence of interdependent relationships between performance and institutional variables in the public sector, further argues that the performance of public organizations is significantly influenced by regulative, normative and cognitive elements. The present study seeks to understand whether resource dependence, particularly dependence on various sources of public funding impacts institutional effectiveness and it argues that due to a complexity of environment in which public higher education organizations exist, Scott's (1995) concept of institutional pillars can be used to explain this dependence.

The role of the government as an institutional actor has multiple meanings in the context of public higher education. Scott (1995) points to the special powers allocated to the state, which give it a unique status among institutional actors and enables it to 
exercise authority over other organizations. It is important to note that Scott uses here the term state in the context of the nation-state, but as Meyer (1994) points out, this authority can be found at different levels of government and the concepts are thus appropriate to use on more than one government level. This authority, according to Scott, permits the state to affect organizations as a collective actor through regulative processes, but also through normative and cognitive processes influencing the institutional structure. For example, “As collective actors", writes Scott (1995), "agencies of the state can take a variety of actions, including granting special charters, allocating key resources such as finance capital or monopoly status, imposing taxes, and exercising regulatory controls" (p. 94).

The existence of multiple authorities and funding sources in public higher education contributes to the fragmentation of its environment, which in turn affects organizational structure of the universities essentially forcing them to develop more complex and elaborate structure in order to deal with this complex, fragmented environment (Scott, 1995). There is no evidence that the impact of these structural changes on organizational effectiveness has been studied except for the indications that these complex structures result, as would be expected, in the growth of the administrative elements of the organization (Scott, 1995).

\section{A Note on Perceptions}

Effectiveness in this study is measured with the information constituencies submitted through the survey and reflects the subjective judgment of these constituencies. Thus, it inevitably relies on the perceptions of the surveyed population and may be 
subject to perceptual bias. Tsui (1990) asserts that this approach "may be appropriate for any social entities that have some sort of constituency relationships, when the entity's effectiveness can only be measured by subjective opinions of some referent group, and/or where consensus does not exist on the relative significance of objective performance measures" (p. 480). Similarly, Schneider, Parkington and Buxton (1980) argue that perceptions of various groups about the organizational practices and procedures represent critical data in examining and understanding organizational behavior. They note that, "No behavior in, or of, organizations occurs in the absence of perceptions" (p. 254).

The relative importance awarded to different constituencies in this study and the influence they potentially can exert on an institution, makes the use of perceptual data not only appropriate but also of special interest for this research. It is also important to note that perceptions can be influenced by a number of factors. Tsui (1990) notes two groups of factors that could influence effectiveness judgment and these are cognitive and social processes involved in perceptions. Obtaining information about constituencies' perceptual judgments can identify potential problems or provide insight about how constituencies differ among each other, which will help concentrate intervention efforts where they are most needed. 


\section{CHAPTER III}

\section{METHOD}

\section{Introduction}

The central issue explored in this study is the relationship between effectiveness and sustainability of public higher education organizations. It has been proposed that the construct of effectiveness is central to sustaining successful operations of higher education institutions. The review of the relevant literature revealed a gap in how effectiveness in higher education is defined and assessed, as well as a need for better understanding of the factors affecting operations and performance of higher education institutions. The purpose of this research is to propose a framework for defining and assessing effectiveness in higher education and examine the role that resources play in effectiveness. The following research questions were used to investigate these tenets: 1. To what extent is organizational effectiveness defined by environmental, social and economic factors as they relate to public colleges and universities?

2. To what degree does resource dependence influence effectiveness of public colleges and universities?

To explore these questions, the operational definitions of effectiveness and resource dependence have been developed. Effectiveness in higher education is defined as creating and maintaining a balance between the economic, social and environmental factors affecting colleges and universities while meeting the needs of their constituencies. 
Resource dependence in this research is defined as dependence on public funding, which includes state funding, federal funding and public financial aid funding.

Based on the research questions, the following hypotheses were tested:

Hypothesis 1a: Perceptions of institutional effectiveness will differ based on the constituency.

Hypothesis 1b: Environmental, social, and economic factors will be strong contributors to organizational effectiveness in higher education.

Hypothesis 1c: Overall institutional effectiveness, and thus sustainability will be higher for institutions that address environmental, social, and economic factors simultaneously than for institutions focusing on only one of these factors at a time.

Hypothesis 2: The influence of resource dependence on institutional effectiveness will differ depending on the predominant sources of public funding.

To examine the proposed questions and hypotheses, this research uses a model in which eleven dependent variables are examined first separately and then clustered in three groups: environmental, social, and economic. ${ }^{1}$ The eleven dependent variables, which demonstrate components of effectiveness in higher education organizations, were used in testing hypotheses $1 \mathrm{a}, 1 \mathrm{~b}$ and $1 \mathrm{c}$. To test hypothesis 2 , the influence of different sources of public funding on effectiveness was examined, taking into consideration the amount of money spent per student and institution type. The next section will describe the

1 The environmental domain of the effectiveness framework refers to the entities, organizations, groups and individuals an organization interacts with and is affected by in some way. The social domain is defined as the internal dimension of an institution and refers to the components such as faculty, administrators and staff workplace satisfaction, morale, organizational culture and health of the institution. The economic domain refers to the ability of an institution to acquire resources it needs, but also the ability to save and use resources effectively. 
research model and show its operationalization in addition to the operationalization of dependent and independent variables.

\section{Research Model}

The theoretical model in Figure 2 was used as a basis for the literature review and in the operationalization of the variables developed for the study. The foundation of this model is the sustainability framework proposed in 1987 by the Brundtland Commission (Cooper and Vargas, 2004), which has as at its core a balance between the economic, social and environmental elements in the society. It is, as Cooper and Vargas (2004) note, "a way of thinking, of living, of governing, and of doing business" (p. 3). Since the study examines the applicability of the sustainability framework as a model for assessing organizational effectiveness in higher education, it is deductive and largely exploratory in its nature. The results of this study will contribute to building a model of effectiveness in higher education and thus will address a gap currently existing in this area. To investigate the research questions, a quantitative approach was employed using data collected from major university constituencies through a survey. 


\section{Figure 2: Theoretical model of effectiveness in higher education}

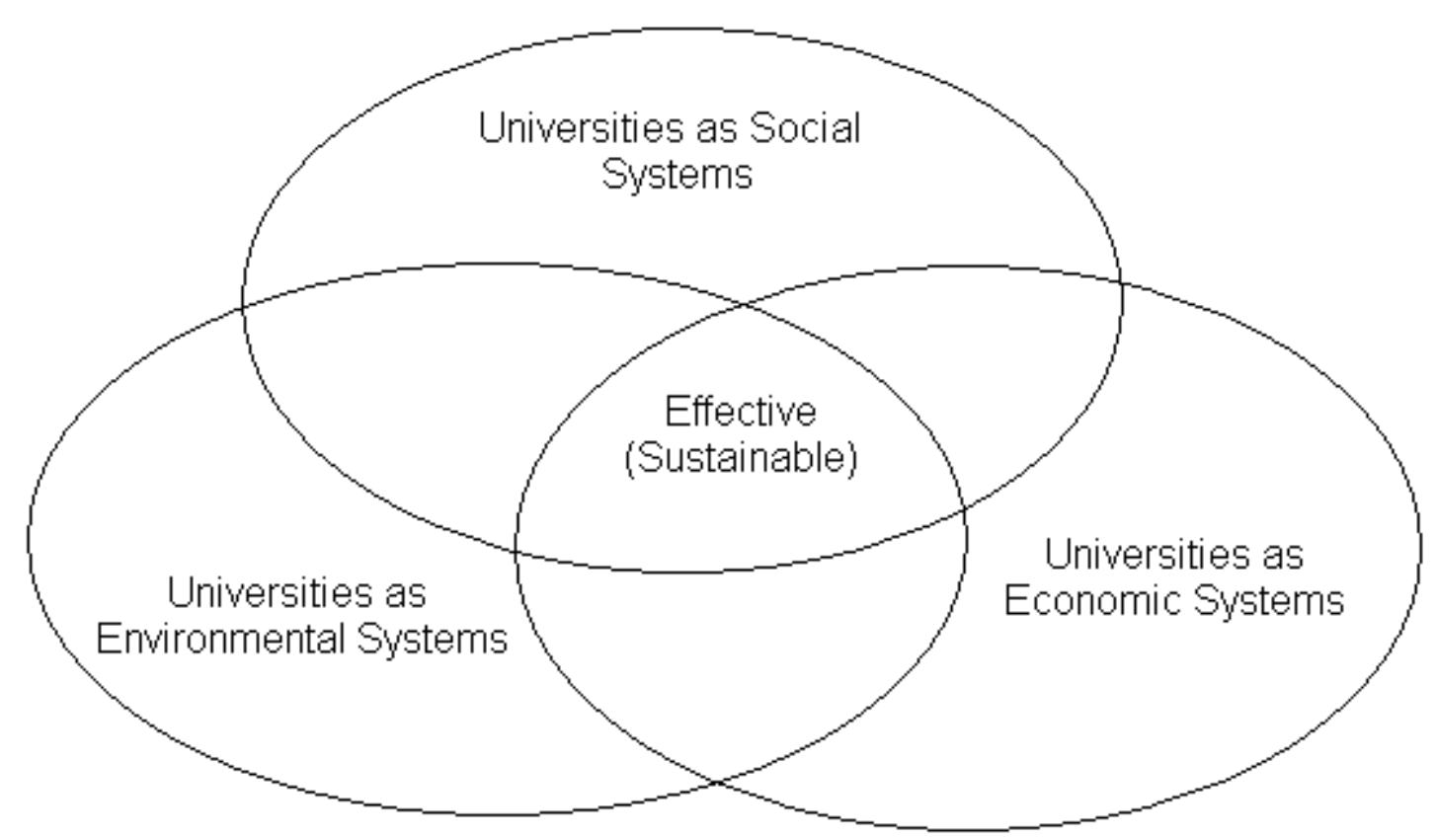

Note: Figure 2 adapted from the Model of Sustainability from the World Conservation Union report: http://cmsdata.iucn.org/downloads/iucn_future_of_sustanability.pdf

The survey instrument consists of a questionnaire reflecting in part the methodology developed by Cameron $(1978,1982)$. The questionnaire consists largely of items developed by Cameron in 1978, but due to specific requirements for this research, it was supplemented with questions taken from the Institutional Performance Survey developed as part of a national research study by the National Center for Higher Education Management Systems (Krakower \& Niwa, 1985). Additional questions were developed by the researcher and some of the existing questions were modified to better reflect the needs of the current research. These modifications addressed an intention to assess institutional conditions as they relate to the environment in which institutions operate their strategy and effectiveness. The added and modified items are intended to 
strengthen Cameron's instrument particularly in the dimensions of financial effectiveness and interaction with environment.

The survey asked respondents to "provide descriptive information, not evaluative judgments, regarding the extent to which their institutions possessed certain characteristics. These characteristics were found in previous research to be indicative of institutional effectiveness" (Cameron, 1982, p. 8). The current study differs from Cameron's in the following important ways:

1. The use of multiple constituencies in determining the effectiveness is broadened to reflect an increased role of external constituencies in the functioning of higher education institutions. Additionally, the group of internal constituencies has been expanded to include faculty and staff, which was not the case in previous studies.

2. The dimensions identified in Cameron's studies were grouped in three domains environmental, social, and economic - and form a basis for the theoretical model (Figure 2). Given that the theoretical model posits, and the literature review (Table 1) revealed additional characteristics not addressed in Cameron's questionnaire, questions were added to the survey to account for missing items. Table 1 shows the three domains as presented in the theoretical model along with groups and factors hypothesized as influencing effectiveness in higher education institutions.

3. This study extends the use of the concept of environment as it is defined in the sustainability framework to the concept of environment in the organizational theory sense of the term. It explores the role of the political and fiscal environment in which institutions of higher education operate. 
4. While Cameron (1986b) did attempt to build a model of effectiveness, the current research aims to test the applicability of the sustainability framework as a model of institutional effectiveness in higher education. The sustainability framework has not been tested in this way before.

\section{TABLE 1}

Factors Influencing Effectiveness in Public Higher Education Institutions Grouped by Domain

Universities as

Environmental Systems

Domain

State Government including members of the State Board of Higher Education

Local Government

Employers/Businesses
Universities as Social Systems Domain

Faculty, staff and administrators: morale, workplace satisfaction, turnover, compensation

Organizational culture
Universities as Economic Systems Domain

Revenues: tuition, state appropriations, auxiliary revenues, increased research funding, gifts, endowments, public/private partnerships, partnerships with local governments Expenditures: accountability, regulations, technology, competition between institutions, operational inefficiencies, administrative lattice and academic ratchet

Students

The principal purpose of this research is to explore to what degree institutional effectiveness allows public colleges and universities to operate in a sustained manner over a long period of time while meeting the needs of their constituencies. The constituencies selected to be included in this study are considered to be relevant to public higher education organizations. Internal constituencies, which in the research model 
comprise social systems domain, consist of faculty, administrators and professional staff. Their importance stems from the fact that they are central to an organizational performance (Van de Ven \& Ferry, 1980) and as such can influence its effectiveness. External constituencies include students, board of higher education members, state and local government officials and employers (businesses and non-profits). These groups are part of the organizational environment and represent the environmental systems domain of the research model.

Regardless of whether the selected constituencies are viewed as internal or external, associated with an organization to a larger or lesser degree, the intent is to "emphasize the possibility that individuals and groups...may form evaluations of its activities, and may be able to influence the activities of that organization to some extent" (p. 213, Connolly, Conlon \& Deutsch, 1980). In some cases, these groups can and, as noted before, do have an effect on organizational performance. In others, they can influence the flow of resources, and yet in third, they can have a role in policy decisions thereby affecting the work of an organization and possibly its effectiveness.

The survey questions operationalize selected dependent variables. According to Singleton, Straits and Straits (1993), "The basic idea of a survey is to measure variables by asking people questions and then to examine the relationships among measures" (p. 254). Table 1 provides a conceptual basis for the study and Table 2 below (page 60) shows the operationalization of the conceptual model depicted in Figure 2 and Table 1. Initial operationalization of dependent variables started with nine dimensions of effectiveness previously identified by Cameron (1978). However, due to added questions, 
it was expected that additional dimensions might be identified. Subsequent analysis confirmed this expectation and three new dimensions related to economic systems domain were identified. Additionally, one of Cameron's dimensions, Student Personal Development, was not identified as a separate dimension. Rather, it was combined with Student Academic Development into a new dimension called Student Academic and Personal Development. Thus, a total of eleven dimensions were identified representing eleven dependent variables used in this research. Details of this process along with factor analysis results and reliability coefficients for each dimension can be found in the next chapter. In order to examine the research questions the eleven dimensions were subsequently grouped in three domains: environmental, social, and economic. The eleven dimensions are operationalized as follows:

1. Student Educational Satisfaction: indicates the degree of student satisfaction with their educational experiences at the institution. The three items developed to measure this variable relate to students satisfaction at the institution and the risk of dropping out due to dissatisfaction with their experience. Respondents were given five-point Likert scale response options ranging from "strongly agree" to "strongly disagree".

2. Student Academic and Personal Development: indicates the extent of student academic attainment, academic and personal growth and progress at the institution. This variable is measured with eight items designed to assess academic attainment of graduates in their major of study, analytical, problem-solving and communication skills, community engagement and social responsibility, presence of a stimulating 
intellectual environment for students, students' engagement in extra academic work, and opportunities institution provides for personal development.

3. Student Career Development: indicates the extent of student occupational development and the opportunities for career development provided by the institution. The four questions designed to assess student career development have a five-point scale ranging from "a large majority" to "a small minority" and relate to benefits of education received in obtaining employment, preparation to compete in the global economy, and students' ability to connect learning with real-world experience. The last question in this group relates to the reason students attend an institution - career or occupational versus social, athletic, financial or other reasons.

4. Capacity to Maintain or Expand Resource Base: indicates the ability to maintain existing or find new sources of funding. The four measures in this dimension relate to the number of potential new students institutions can recruit, ability to expand the array of academic programs, increasing the number of admitted out-of-state students, and the ability to establish new domains of activity. The response options for these items range from "strongly agree" to "strongly disagree".

5. Faculty, Administrator and Staff Employment Satisfaction: measures the satisfaction by faculty members and administrators with their job and employment at the institution. This variable is assessed on a five-point scale with responses ranging from "a large majority" to "a small minority" and includes six items asking faculty, administrators and staff about their personal satisfaction with employment and probability of leaving if given the opportunity. 
6. Professional Development and Quality of the Faculty: evaluates the extent of faculty professional attainment and development and the amount of professional development support provided by the institution. This variable is measured with four items on a five-point scale with responses ranging from "a large majority" to "a small minority". The questions in this group are intended to obtain information about the number of faculty publishing books/articles, displaying work of art, receiving awards, teaching at a "cutting edge", engaging in research or external consulting.

7. System Openness and Interaction: indicates the emphasis placed on interaction with, adaptation to, and service in the external environment. This set of nine questions measures the degree to which an institution is open and interacts with its environment. These questions ask how responsive and adaptive an institution is to external constituencies, the degree to which faculty and administrators serve on boards, committees and other bodies outside of the university, the emphasis on institution-community and community oriented programs and projects sponsored by the institution, focus on research partnerships with public and private entities, and the institution's impact on the economic development of the region. In addition, this variable is assessed using questions about faculty and administrators engagement in activities outside of the institution, investment in functions dealing with external groups (e.g., admissions, development, government relations, businesses), institution's openness to new activities and policies and whether institutional members educate important outsiders about the value of the institution. 
8. Resources for Quality Programs, Faculty and Students: assesses the ability of the institution to acquire resources from the external environment and to attract high quality students and faculty. The five questions in this part are designed to obtain respondents' level of agreement with the statements about an ability of the institution to obtain financial resources to provide quality instructional programs, as well as resources it needs to be effective, an ability to attract high achieving students, to attract faculty who are leaders in their fields, and to establish an alumni base committed to the institution. Responses in this section range from "strongly agree" to "strongly disagree" on a five-point Likert scale.

9. Organizational Culture and Health: indicates the extent of smooth functioning of the institution and vitality and viability in its internal processes and practices. This set of eight items relates to measuring organizational culture and health and includes questions about student/faculty relationships, equity of treatment and awards among employees, interdepartmental relations, general pattern of supervision and control, general health of the institution (smooth, productive functioning), recognition and reward received from superiors, the amount of information or feedback received, and general social environment.

10. Ability to Acquire, Save and Use Resources Effectively: the four items in this dimension measure institutional emphasis on finding new sources of funding, but also on containing costs and using resources effectively. In addition, it inquires about the competing power of the institution to recruit top students. 
11. Ability to Overcome Financial Difficulties: the two items in this dimension ask about external uncertainties affecting revenues and difficulties in obtaining financial resources. This variable is measured with responses ranging from "strongly agree" to "strongly disagree" on a five-point scale.

\section{TABLE 2}

Summary of Operationalized Dependent Variables Grouped by Domains

Universities as

Environmental Systems

Domain

Student Educational

Satisfaction (1)

Student Academic and

Personal Development (2)

Student Career

Development (3)

System Openness and Interaction (7)
Universities as Social

Systems Domain

Faculty, Administrator and

Staff Employment

Satisfaction (5)

Professional Development

and Quality of the Faculty

(6)

Organizational Culture and

Health (9)
Universities as Economic Systems Domain

Capacity to Maintain or Expand Resource Base (4)

Resources for Quality Programs, Faculty and Students (8)

Ability to Acquire, Save and Use Resources Effectively (10)

Ability to Overcome Financial Difficulties (11)

The survey instrument includes items designed to measure participants' perceptions about the eleven dimensions of effectiveness noted above. Given variations in the level of familiarity with institutional processes among the constituencies surveyed, the decision was made to employ three surveys, one for university employees, one for students and one for other external constituencies. The university employees responded to the full version of the survey which contained the highest number of questions, while 
some of the questions were omitted for students and external groups. Using three versions of the survey is considered appropriate since there was no expectation that external constituencies would know details about internal institutional processes. For example, business leaders and employers in general would not have enough information about faculty, administrator and staff employment satisfaction, making this question irrelevant to external constituencies. The survey was piloted with a convenience sample involving members of each constituency with the purpose to identify potential problems and refine the survey instrument. Based on the results of the pilot, some questions were modified for clarity and consistency.

The three versions of the survey instrument, which appear in Appendices B, C and $\mathrm{D}$, were developed with each dimension containing between two and nine items related to the criteria measuring these dimensions. To obtain effectiveness scores for each dimension, the eleven dimensions were first analyzed individually and mean scores for each dimension were computed. Next, the eleven dimensions were grouped in three domains noted in Table 2 and the results for all dimensions, domains and overall effectiveness were used to test hypotheses and the theoretical model of effectiveness proposed in this research.

The Environmental Systems Domain consists of four dimensions containing twenty-four items in the domain. The Student Educational Satisfaction is measured with items 1.8 to 1.10, while the Student Academic and Personal Development is measured with items $1.1,2.1,2.2,2.3,4.1,4.2,4.3$ and 7.1 . The items 7.2 to 7.5 measure Student Career Development. The following items measure the variable of System Openness and 
Interaction: $1.2,4.4,4.5,4.6,4.7,5.5,9.3,9.4$ and 9.5. Table 28 in Appendix E shows the eleven effectiveness dimensions and the relationship between individual items and each dimension. The Social Systems Domain consists of three dimensions containing eighteen items. The Faculty, Administrator and Staff Employment Satisfaction is measured with items 3.1 to 3.6 and Professional Development and Quality of the Faculty is measured with items 5.1 to 5.4. The items 6.1 to 6.8 illustrate measures for Organizational Culture and Health dimension. The Economic Systems Domain consists of four dimensions containing fifteen items. Items 8.2, 9.1, 9.2 and 9.6 measure the Capacity to Maintain or Expand Resource Base, while Resources for Quality Programs, Faculty and Students are measured with items 1.3 to 1.7 . The Ability to Acquire, Save and Use Resources Effectively dimensions consists of items 5.6, 9.7, 9.8 and 9.9. The last dimension in this group, Ability to Overcome Financial Difficulties, consists of two items 8.1 and 8.3.

The independent variables are the level of dependence on public funding, broken down to state funding, federal funding and public financial aid funding. These variables were measured using institutional budget data for the fiscal year 2009-10. More specifically, state funding includes all operational funding provided to a university by the state, including state fiscal stabilization funding (ARRA). Federal funding consists of all federal grants received, which for the most part consists of funding for research as well as a small amount coming from the fiscal stabilization fund related to federal grants. Lastly, public financial aid variable consists of funding from Pell grants, other federal financial aid grants, grants from state and local governments as well as institutional fee remissions. 
In addition, two other independent variables were used in the study, spending per student FTE and institution type. According to Ehrenberg (2007), the spending per student in public higher education has declined in recent times, and while the relationship between spending and quality of education has not been clearly established, the concerns about the decline in the quality of public higher education due to a decrease in expenditures per student should not be ignored. The Delta Cost Project report (2009) confirms the trends in spending per student, noting that the cost per degree has declined in public higher education in recent years. The report voices similar concerns about the quality, albeit warning that no conclusions can be made without established quality benchmarks.

The information about spending per student FTE was obtained from the institutional data. It was calculated by combining institutional expenditures and the amount allocated to instruction from other program areas, such as academic support, student services, plant operation and maintenance, and institutional support. This calculation is based on the relative amount each institution spends on instruction, research, public service and other activities. The obtained total amount spent on instruction was then divided by the number of full time equivalent students at each institution, resulting in spending per student FTE amount used as independent variable. The universities involved in the study are categorized as institutions with comprehensive research and urban missions. Since each institution has a unique public funding mix based on their mission, the expectation was that the funding mix and amounts could potentially have an influence on their effectiveness. 


\section{Sample and Data Collection}

Public four-year institutions of higher education from the same state were recruited for this study. The identified universities were approached and asked to participate, two of them agreed. The reasons this sampling procedure was chosen were threefold. First, given the intent to explore the link between effectiveness and resource dependence, this sample allowed for insight about how different public institutions within the same state context address the issue of resource dependence and whether it has an influence on their effectiveness. It is proposed that even though the sample institutions are public institutions from the same state system, not all of them will depend on public resources to the same degree. Thus, their effectiveness may vary in one or more domains.

Second, this sample allowed examination of the role of multiple constituencies in determining the effectiveness of institutions. For each of the institutions, members of four groups of constituencies, comprising six distinct constituencies, were asked to provide information through a survey administered online. The selected constituencies included administrators (executive, academic affairs, financial affairs, student affairs, deans and department chairs), faculty, professional staff, and major external constituencies who can be and often are major funders as well. The category of major external constituencies consisted of students, government members (board of education, state legislators, local government officials), and a group of employers (businesses, non-profit organizations) employing graduates of a particular university. The preference to explore the utility of multiple constituencies in defining effectiveness and the large number of constituencies surveyed contributed to the decision to limit the study to institutions within one state. The 
role of various constituencies is especially relevant in today's environment where the calls for an increased accountability in higher education can be heard from a number of sources, not the least from the groups included in this study. At the same time, not less important is a need to improve internal functioning of colleges and universities as seen by faculty, staff and administrators.

Third, as noted before, one of the research questions examines whether organizational effectiveness in higher education can be defined by environmental, social, and economic factors affecting colleges and universities, testing thereby the applicability of sustainability framework as a model of effectiveness. Thus, the exploratory nature of this inquiry deems the use of non-probability sample appropriate. According to Singleton, Straits and Straits (1993), the use of purposive sampling is justified in cases where the researcher wants to acquire more information about the problem without intention to produce precise statistical generalization. Biemer and Lyberg (2003) note that purposive sampling, also called judgment sampling, relies on the researcher's expert judgment to select cases representing the intended population, adding that this kind of sample, if designed properly, can have characteristics of the general population. Further, as Biemer and Lyberg assert, purposive sampling with a very small sample can provide more accurate information than random sampling. In addition, sampling error could potentially be much larger with a random sample of this size than with purposive sample.

Selecting a sample size needs to take into consideration significance criterion $(\alpha)$, population effect size (ES) and statistical power (1- $\beta$ ). As Cohen (1992) notes, the relationship among these four variables is crucial in statistical power analysis and each of 
them is a function of the other three. The significance criterion $\alpha$ is conventionally taken to be .05 , which is a compromise between minimizing Type I error and maximizing power. Type I error happens "when the populations that underlie a study show no effect, but the samples in the study accidentally show an effect large enough that a statistical test yields a (falsely) significant result" (p. 122, Cohen \& Lea, 2004).

According to Cohen, Cohen, West \& Aiken (2003), failing to control for statistical power can result in Type II error, which is "the error of failing to reject false null hypotheses and failing to find things that are there" (p. 183). The conventional value recommended for power is .80 , and it represents the middle ground between committing Type II error and having too large sample size. Effect size is often noted as the most difficult part to be determined in the power analysis. Effect size represents the degree to which null hypothesis is false (Cohen, 1992), and Cohen's guidelines for effect size are established conventions used in power analysis. Table 3 presents Cohen's effect size guidelines (1992) used in one-way analysis of variance and were used in this research to determine the sample size for the populations under study.

\section{TABLE 3}

Guidelines for Effect Size

\begin{tabular}{lccc}
\hline & Small & Medium & Large \\
\hline Effect Size & .1 & .25 & .4 \\
\hline
\end{tabular}

As Cohen (1992) notes, in planning the research, it is important to know sample size needed to achieve the desired power for specified effect size and significance level. This is even more important in the current study given the number of groups to be surveyed and the predetermined size of at least two groups. Table 4 shows the minimum 
sample size required for each group to achieve power of .80 at the .05 significance level for small, medium and large effect size based on Cohen's (1992) recommendations.

\section{TABLE 4}

Sample Size Guidelines for Six Groups Based on Effect Size at $\alpha=.05$ and Power $=.80$ Effect Size

\begin{tabular}{lccc} 
& Small (.1) & Medium (.25) & Large (.4) \\
\hline Sample Size & 215 & 35 & 14 \\
\hline
\end{tabular}

A web-based survey was administered to selected populations for the two participating institutions. As Singleton, Straits and Straits (1993) note, "surveys offer the most effective means of social description; they can provide extraordinarily detailed and precise information about large heterogeneous population" (p. 252). Faculty, administrators and staff from each university were surveyed. The faculty group included full-time faculty (tenured, tenure-track and fixed term), while the administrators' group comprised of executive, academic affairs, financial affairs, and student affairs administrators as well as deans and department chairs. Although the staff category consists of a number of different positions, only professional staff working in student services and support services was included in this study. This decision was made due to a difference in categorization of positions at various universities, which would make the comparisons difficult.

The institutions selected to participate in the study were chosen through a purposive sampling procedure and the sampling of the population for each university depended on a constituency and involved either random or purposive sampling. Due to a request from one of the institutions to limit the sample size originally planned and due to 
the fact that this request came a few days after the survey started at another institution, the sampling procedure for two institutions differed with some of the constituencies. Thus, the sample of faculty, administrators and staff at Institution 1 was obtained by census of employees in the categories noted above, while the sample at Institution 2 included a percentage of total number of employees. This percentage was drawn by simple random sampling procedure from the list of all full time employees in academic and non-academic departments that belonged to one of the three constituencies.

The next group included in the survey was external group consisting of government groups including board members, state legislators and local government officials. Since the institutions involved in the research belong to the same state system, all board members were included in the survey for both universities. The same procedure was followed for state legislators, a group which included members of the Senate and House education committees. A purposive sample of local government officials in the area where each university is located was drawn from the list of local government agencies. The second external group included in the survey was a group of employers recruiting graduates of the institution under study. This group included businesses as well as non-profit organizations in the area where each university is located and beyond. The list of participants in this group was obtained through the institutional student career offices and their websites using purposive sampling procedure. Lastly, the students' sample at both institutions was drawn randomly from all students with senior class standing at the time of survey administration. 
Using Cohen's guidelines listed in Table 4, minimum acceptable sample size was selected for each constituency studied. However, given that the government and employers had either a predetermined size or were limited in size due to constraints in obtaining information about sample participants, unequal samples were drawn. Cohen (1988) states that unequal sampling is acceptable and occurs in certain circumstances such as in studies where one or more groups is fixed in size and the size of a sample is left to the researcher to decide. Thus, the survey response for four groups - faculty, administrators, professional staff and students - needed to be a minimum of 35 for each group for medium effect size, while government and employers needed at least 14 respondents for large effect size. This goal was achieved for all groups except for employer group at Institution 2. In order to remediate this problem, the decision was made to combine two external groups, government and employers where needed. This decision changed in some cases the number of groups included in the analysis from six to five, which consequently changed the minimum sample size needed for each constituency from 35 to 39 for medium effect size and 14 to 16 for large effect size. Nevertheless, the sampling procedure described satisfies power analysis criteria established by Cohen and presented in Table 5.

An initial e-mail was sent to the participants notifying them about the upcoming online survey and inviting them to participate (Appendix A). This e-mail also contained brief information about the study and identified the sender of the e-mail as well as the subject of the forthcoming e-mail. The e-mail with the survey link was sent one week after the initial e-mail (Appendix A). In addition, two follow up e-mails, a week apart 
from each other, were sent to non-responders (Appendix A). The survey was administered over a period of one month during the fall of 2010 .

The survey sample consisted of 2,694 employees, 151 government representatives, 1,090 employers and 5,300 students from the two universities. The response rate was $19.1 \%$ for employees, $18.5 \%$ for government, $9.9 \%$ for employers and 8.8\% for students. A look at the response rate for each institution shows that internal constituencies were more likely to respond to the survey than external ones including the students, which was an expected result. In addition, there was not much variation in the response rates between institutions, except for employees, where faculty, administrators and staff at Institution 1 had higher response rate than the same population at the Institution 2. Table 5 presents constituencies surveyed, sample sizes for each of the four groups, and the number of respondents and response rate for each constituency by institution as well as combined. 


\section{TABLE 5}

Study Population and Response Rate by Constituency and by Institution

\begin{tabular}{|c|c|c|c|}
\hline Constituency & Sample (N) & Respondents (n) & Response Rate \\
\hline Total & 9235 & 1119 & $12.1 \%$ \\
\hline Employees & 2694 & 515 & $19.1 \%$ \\
\hline Faculty & & 227 & \\
\hline Administrators & & 83 & \\
\hline Professional Staff & & 120 & \\
\hline External: Government & 151 & 28 & $18.5 \%$ \\
\hline External: Employers & 1090 & 108 & $9.9 \%$ \\
\hline Students & 5300 & 468 & $8.8 \%$ \\
\hline \multicolumn{4}{|l|}{ Institution 1} \\
\hline Total & 5888 & 783 & $13.3 \%$ \\
\hline Employees & 1727 & 377 & $21.8 \%$ \\
\hline Faculty & & 169 & \\
\hline Administrators & & 63 & \\
\hline Professional Staff & & 95 & \\
\hline External: Government & 88 & 19 & $21.6 \%$ \\
\hline External: Employers & 1000 & 97 & $9.7 \%$ \\
\hline Students & 3073 & 290 & $9.4 \%$ \\
\hline \multicolumn{4}{|l|}{ Institution 2} \\
\hline Total & 3347 & 341 & $10.2 \%$ \\
\hline Employees & 967 & 138 & $14.3 \%$ \\
\hline Faculty & & 58 & \\
\hline Administrators & & 20 & \\
\hline Professional Staff & & 25 & \\
\hline External: Government & 63 & 14 & $22.2 \%$ \\
\hline External: Employers & 90 & 11 & $12.2 \%$ \\
\hline Students & 2227 & 178 & $8 \%$ \\
\hline
\end{tabular}

\section{Method of Data Analysis}

Prior to conducting data analysis, some negatively worded items first needed to be reverse coded. A total of eight items was reverse coded, two related to Student Educational Satisfaction, three related to Faculty, Administrators and Staff Employment Satisfaction, two items asking about the institutional Ability to Overcome Financial 
Challenges and one item related to Organizational Culture and Health of the institution. Next, exploratory factor analysis was performed to reduce a number of newly created variables into factors called dimensions of effectiveness, but also to determine whether Cameron's dimensions are valid for this research due to the additional items. After checking for reliability and validity of dimensions, a number of statistical procedures were performed to test the research questions and hypotheses.

To examine research question one, which inquired about the extent to which organizational effectiveness is defined by environmental, social, and economic factors as they relate to public colleges and universities, and related hypotheses, a number of analysis of variance (ANOVAs) were performed. The purpose of conducting the first series of ANOVAs was to test hypothesis 1a which looked at the differences in ratings of effectiveness among constituencies within each institution by dimension. To address hypotheses $1 \mathrm{~b}$ and $1 \mathrm{c}$, additional ANOVAs were conducted with the goal to determine whether there is a difference in the ratings of environmental, social, and economic effectiveness domains within each institution as well as in the overall effectiveness. These procedures, in addition to factorial ANOVAs discussed below, were useful in testing the hypotheses $1 \mathrm{a}, 1 \mathrm{~b}, 1 \mathrm{c}$, and the overall research model and in determining the degree to which the three domains interact and the way in which they interact.

To test research question two, which asked about the influence of dependence on state, federal and public financial aid funding, and spending per student FTE on effectiveness, a two-pronged approach was used. First, a series of two-way analysis of variance or factorial ANOVAs were conducted with institution type and constituency as 
independent variables and the eleven dimensions of effectiveness, the three domains and overall effectiveness as dependent variables. Factorial ANOVAs were conducted to determine the influence of institution type and constituency as well as to test for possible interaction between the two on each dependent variable noted above.

The second step in examining research question two involved first looking at patterns in the level of funding based on the funding source for each institution and then comparing the means for each dimension, domain and overall effectiveness between the two institutions. To that end, independent sample t-tests were performed for each dependent variable. These analyses provided answers in testing hypothesis 2 , which states that resource dependence influence on institutional effectiveness will differ depending on the predominant source of public funding.

\section{Limitations of the Method}

There are several limitations to this methodology. First, even though certain population categories were chosen randomly, the sample of universities selected to participate in this study was not chosen randomly which can limit the generalizibility of the research results. Biemer and Lyberg (2003) note that random sampling significantly reduces sampling bias. However, as noted before, the sampling error would be large with this small number of institutions and can be reduced with purposive sampling. Thus, the trade off that had to be made to fulfill the purpose of the research is considered appropriate. Second, since some of the constituencies are small in number either by their nature or due to difficulties in obtaining a larger sample (e.g., legislative committees, employers), their size required some modification in the analysis stage of the research. To 
minimize the negative effect of the variation in size and consequent smaller response rates, some constituencies needed to be combined for one of the institutions.

Third, the study relies on perceptions collected from the populations surveyed. There is no objective measurement of the variables examined. Further research could address this by measuring the identified variables with the objective data, provided they exist for each institution. Moreover, it is a cross-sectional study, providing a snapshot of the effectiveness for the institutions studied. Singleton, Straits and Straits (1993) note that, "Because cross-sectional designs call for collection of data at one point in time, they do not always show clearly the direction of causal relationships and they are not well suited to the study of process and change" (p. 255). One of the ways to address this problem is to use a longitudinal design in which data is collected at different points in time. Given that it is not always possible to undertake a longitudinal study, another way to address this limitation is to have questions in the survey that account for the study of process or change (Babbie, 1973).

Fourth, due to a small number of cases (two institutions) involved in the study, research questions related to dependence on various public funding sources can only be partly answered. It does, however, provide a pathway for more robust future research which would involve a larger number of institutions. Despite these limitations, this study represents an important contribution to the field of organizational effectiveness and the role resources play in effectiveness, as well as a contribution to organizational theory in general. 


\section{Summary}

This research was designed to explore the role of institutional effectiveness and resource dependence in attaining a sustainable future of public colleges and universities while meeting the needs of their constituencies. It has been proposed that in order for institutions to be considered effective, there needs to exist a balance in addressing economic, social and environmental factors affecting them. A quantitative approach using an online survey was employed to examine the research questions and to test the hypotheses put forward in the study. The next chapters will address the results of the study and its implications for future research and practice. 


\section{CHAPTER IV}

\section{RESULTS}

The proposed research relied primarily on quantitative research techniques to analyze data collected via surveys. It was expected that the reliance on multiple dimensions of effectiveness examined individually as well as grouped in three domains (environmental, social, and economic $)^{1}$ and involvement of multiple constituencies would increase reliability and internal validity of the research. The data analysis follows and extends the analysis Cameron performed in a number of studies $(1978,1981,1982)$.

The data analysis involved a number of separate procedures. After cleaning the data, it was examined for skewness and kurtosis and existence of any outliers. According to Field (2009), skewness and kurtosis will be above or below zero if the scores deviate from a normal distribution. Thus, the more normally distributed scores are, the closer they are to zero. Field recommends that skewness and kurtosis values should not exceed 2, but he notes that it also depends on sample size and the larger the sample the higher this value can be. Upon data examination, it was determined that there were no values above 2, and all except one were below 1, indicating that there are no problems with the data in this regard. Data was also checked for outliers and outliers were identified for one item. After examining skewness and kurtosis for this item, as well as its histogram and

\footnotetext{
1 The environmental domain of the effectiveness framework refers to the entities, organizations, groups and individuals an organization interacts with and is affected by in some way. The social domain is defined as the internal dimension of an institution and refers to the components such as faculty, administrators and staff workplace satisfaction, morale, organizational culture and health of the institution. The economic domain refers to the ability of an institution to acquire resources it needs, but also the ability to save and use resources effectively.
} 
boxplot it was determined that there is no reason to remove this item from the data set, thus the decision was made to keep it.

Next, psychometric tests were conducted to ensure that the dimensions of effectiveness have validity and reliability. Cameron $(1978,1982)$ found that the nine dimensions he developed did have both, high reliability and validity, however given the addition of questions in the instrument used in this research, it was important to test for it in this study. To that end, a series of exploratory factor analytic procedures were employed to check on the construct validity and to "determine the dimensionality of the intended scales" (p. 11, Clott, 1995). In addition, the reliability of the items was assessed by computing Cronbach's alpha on all the items in the survey. The use of factor analysis is considered appropriate when the purpose is to reduce the number of variables to a smaller number of factors. As Babbie (1973) notes, "It is an efficient method for discovering predominant patterns among a large number of variables" (p. 328).

\section{Factor Analysis}

Exploratory factor analysis was performed using principal axis factoring extraction method with oblique rotation. Oblique rotation method used was promax rotation with Kaiser normalization. Kaiser-Meyer-Olkint test was run to test for sampling adequacy and resulted in $\mathrm{KMO}=.90$, which is a superb value according to Field (2009). In addition, Bartlett's test for sphericity was significant $(\mathrm{p}<.001)$ indicating that factor analysis was appropriate in this case. Field (2009) recommends looking at a sample size, eigenvalues and scree plot to identify an appropriate number of factors. It is generally recommended that factors with eigenvalues above 1 are retained, especially with large 
samples as it is in this case. The scree plot can also be used as a guide and the usual cutoff point is where the curve levels off after a steep drop. However, Field also notes that in some cases the results of the scree plot can be ambiguous because there may be more than one place where the curve drops, although usually not as dramatically as the first drop. Ultimately, factor analysis resulted in identifying eleven factors accounting for 49.6\% of the variance. While Cameron identified nine factors in his research, it was expected that current research might reveal more factors due to the added questions, especially questions related to financial conditions of institutions. Three additional financial factors besides one factor previously identified by Cameron were indeed extracted resulting in four total financial dimensions.

One of Cameron's dimensions, Student Personal Development, did not load on a separate factor. Rather, the three questions from this dimension loaded onto the Student Academic Development dimension. Thus, the decision was made to combine these two dimensions into a new dimension called Student Academic and Personal Development. Additionally, one question, "After students leave this institution, they maintain a strong commitment to the institution" originally belonging to Student Educational Satisfaction did not load on this dimension. Rather, it loaded on two other factors, Student Academic Development and Resources for Quality Programs, Faculty and Students. Given that commitment of the graduates to the institution can, and often does, result in alumni giving, the decision was made to place it under financial dimension - Resources for Quality Programs, Faculty and Students. It is worth noting that some questions loaded on more than one factor, some with very close factor loadings. After careful consideration of 
the goals of the current research, in most cases the decision was made to keep them consistent with Cameron's dimensions.

Two items did not load on any factor and were removed from the data set. The factor analysis was re-run without these two items and the results were consistent with results before removing the items. The removed questions were: "Factors outside the institution that affect its enrollments have become more predictable over the last five years" and "This institution tries to insulate itself from demands and pressures in the environment". The first question was related to one of the financial dimensions, while the second question belonged to system openness and interaction dimension.

Since there were three versions of the survey used in this research, for employees, students and external constituencies, factor analysis was run with each group separately as well as with all groups combined. The results were consistent, with the reminder that students and external groups did not have all questions and all dimensions represented in their questionnaire. Given these consistent results and the intention to compare results among constituencies, the decision was made to run factor analysis with one combined data set. The final eleven factors identified with factor analysis are: Student Educational Satisfaction, Student Academic and Personal Development, Student Career Development, Capacity to Maintain or Expand Resource Base, Faculty, Staff and Administrator Employment Satisfaction, Professional Development and Quality of the Faculty, System Openness and Interaction, Resources for Quality Programs, Faculty and Students, Organizational Culture and Health, Ability to Acquire, Save and Use Resources Effectively; and Ability to Overcome Financial Difficulties. 
Cronbach's alpha was computed to test for reliability and consistency of the dimensions and results for each but two dimension were in the range between .70 and .84 indicating good overall reliability (Field, 2009). The Capacity to Maintain or Expand Resource Base dimension had Cronbach's $\alpha=.55$ and Ability to Acquire, Save and Use Resources Effectively had $\alpha=.57$. Field (2009) notes that literature considers Cronbach's alpha .70 to .80 as acceptable value. Thus, the two values above would indicate relatively low reliability. However, Field also notes that some researchers such as Kline consider values below .70 acceptable depending on the type of construct being studied. In this case, one possible explanation could be that concepts covered in the two dimensions are more different or less consistent than expected and they deserve a second look in any subsequent study. Table 6 lists factor loadings for each item and Cronbach's coefficient alpha for each dimension.

\section{TABLE 6}

Dimensions of Effectiveness, Item Loadings and Reliability Values

\begin{tabular}{lcc} 
Factor and Individual Item Measures & Loading & $\alpha$ \\
\hline 1. Student educational satisfaction (SEDS) & .63 & .75 \\
\hline Satisfaction among students & .66 & \\
\hline $\begin{array}{l}\text { Students drop out because of dissatisfaction with } \\
\text { educational experiences }\end{array}$ & .61 & .84 \\
\hline$\quad$ Students complain about educational experiences & .54 \\
\hline 2. Student academic and personal development (SAPD) & .73 \\
\hline Opportunity for personal development & .75 \\
\hline $\begin{array}{l}\text { Acquisition of analytical, problem-solving and } \\
\text { communication skills }\end{array}$ & .44 \\
\hline $\begin{array}{l}\text { Depth of knowledge in major of study } \\
\text { Community engagement and social responsibility }\end{array}$ & .39 \\
\hline $\begin{array}{l}\text { Aevelopment } \\
\text { academic development }\end{array}$
\end{tabular}




\begin{tabular}{|c|c|c|}
\hline Students develop and mature in non-academic areas & .56 & \\
\hline $\begin{array}{l}\text { Stimulating intellectual environment for student } \\
\text { academic development }\end{array}$ & .70 & \\
\hline Students engaging in extra academic work & .55 & \\
\hline 3. Student career development (SCAD) & & .74 \\
\hline $\begin{array}{l}\text { Education graduates received beneficial in obtaining } \\
\text { employment }\end{array}$ & .58 & \\
\hline $\begin{array}{l}\text { Graduates prepared for challenges of global } \\
\text { economy }\end{array}$ & .77 & \\
\hline $\begin{array}{l}\text { Graduates able to connect classroom learning with } \\
\text { real world experience }\end{array}$ & .75 & \\
\hline $\begin{array}{l}\text { Students attending institution for career or } \\
\text { occupational goals as opposed to social, athletic, } \\
\text { financial, or other reasons }\end{array}$ & .42 & \\
\hline 4. Capacity to maintain or expand resource base (CMER) & & .55 \\
\hline Potential students institution can recruit & .32 & \\
\hline Expanding array of academic programs & .61 & \\
\hline Increasing number of out-of-state students & .46 & \\
\hline Institution establishes new domains of activity & .70 & \\
\hline $\begin{array}{l}\text { 5. Faculty, staff and administrator employment } \\
\text { satisfaction (FAES) }\end{array}$ & & .84 \\
\hline $\begin{array}{l}\text { Faculty leaving for another job for professional } \\
\text { reasons }\end{array}$ & .71 & \\
\hline $\begin{array}{l}\text { Administrators leaving for another job for } \\
\text { professional reasons }\end{array}$ & .72 & \\
\hline Staff leaving for another job for professional reasons & .74 & \\
\hline Faculty personally satisfied with employment & .62 & \\
\hline Administrators personally satisfied with employment & .58 & \\
\hline Staff personally satisfied with employment & .57 & \\
\hline $\begin{array}{l}\text { 6. Professional development and quality of the faculty } \\
\text { (PDQF) }\end{array}$ & & .84 \\
\hline $\begin{array}{l}\text { Faculty publishing books or articles or displaying } \\
\text { work of art in a show }\end{array}$ & .75 & \\
\hline Faculty teaching at a "cutting edge" in their field & .72 & \\
\hline $\begin{array}{l}\text { Faculty engaged in activities such as research, } \\
\text { consulting, getting an advanced degree, etc. }\end{array}$ & .80 & \\
\hline $\begin{array}{l}\text { Faculty receiving academic or professional awards, } \\
\text { honors, etc. }\end{array}$ & .70 & \\
\hline 7. System openness and interaction (SOCI) & & .80 \\
\hline $\begin{array}{l}\text { Institution responsive and adaptive to external } \\
\text { constituencies }\end{array}$ & .56 & \\
\hline Faculty, administrators and staff engage in & & \\
\hline
\end{tabular}




\begin{tabular}{|c|c|c|}
\hline professional activities outside the institution & .55 & \\
\hline $\begin{array}{l}\text { Institution sponsors community-oriented programs, } \\
\text { workshops, projects, or activities }\end{array}$ & .69 & \\
\hline Institution creates research partnerships & .63 & \\
\hline $\begin{array}{l}\text { Institution has positive impact on economic } \\
\text { development }\end{array}$ & 61 & \\
\hline $\begin{array}{l}\text { Faculty, administrators and staff serving in } \\
\text { government, on boards and committees, etc. }\end{array}$ & .40 & \\
\hline $\begin{array}{l}\text { Investment in outreach functions dealing with } \\
\text { external people (admissions, development, } \\
\text { government relations, etc.) }\end{array}$ & .41 & \\
\hline Openness for new activities and policies & .39 & \\
\hline $\begin{array}{l}\text { Institutional members educate important outsiders } \\
\text { about the value of institution }\end{array}$ & .53 & \\
\hline $\begin{array}{l}\text { 8. Resources for quality programs, faculty and students } \\
\text { (QPFS) }\end{array}$ & & .82 \\
\hline $\begin{array}{l}\text { Institution can obtain financial resources for quality } \\
\text { instructional programs }\end{array}$ & .86 & \\
\hline $\begin{array}{l}\text { Institution can obtain resources it needs to be } \\
\text { effective }\end{array}$ & .94 & \\
\hline Institution can attract leading faculty in their field & .55 & \\
\hline Institution can attract leading high school graduates & .53 & \\
\hline $\begin{array}{l}\text { Students maintain commitment to institution after } \\
\text { leaving }\end{array}$ & .51 & \\
\hline 9. Organizational culture and health $(\mathrm{ORCH})$ & & .84 \\
\hline Student/faculty relationships & .45 & \\
\hline Equity of treatment and rewards & .64 & \\
\hline Interdepartmental relations in the institution & .64 & \\
\hline General pattern of supervision and control & .35 & \\
\hline Organizational health of the institution & .74 & \\
\hline $\begin{array}{l}\text { Recognition and reward received for good work } \\
\text { from superiors }\end{array}$ & .70 & \\
\hline The amount of information or feedback received & .77 & \\
\hline The general social environment & .77 & \\
\hline $\begin{array}{l}\text { 10. Ability to acquire, save and use resources effectively } \\
\text { (AACR) }\end{array}$ & & .57 \\
\hline $\begin{array}{l}\text { Proportion of top students attending institution rather } \\
\text { than competition }\end{array}$ & .53 & \\
\hline Administrators emphasizing finding new money & .52 & \\
\hline Administrators emphasizing saving money & .46 & \\
\hline $\begin{array}{l}\text { Administrators provide incentives for conserving } \\
\text { resources }\end{array}$ & .53 & \\
\hline
\end{tabular}




\begin{tabular}{ccc}
\hline 11. Ability to overcome financial difficulties (AOFD) & & .70 \\
\hline External uncertainties affecting revenues & .81 & \\
\hline Difficulty of obtaining financial resources & .57 \\
\hline
\end{tabular}

To examine the relationship among the dimensions, correlation coefficients were computed for all eleven dimensions (Table 7). The results indicate that there is moderate to low and even negative correlation among eleven dimensions ranging from -.09 to .55 . These correlations suggest that dimensions can be differentiated from one another. Results also show absence of very high correlations $(r>.8)$, indicating that there are no problems with multicollinearity (Field, 2009). While it does not necessarily mean it is problematic, the negative correlation of the Ability to Overcome Financial Difficulties dimension with four other dimensions deserves further exploration, such as looking at the wording of the items or considering the fact that this dimension consists of only two items.

\section{TABLE 7}

Correlations Among Effectiveness Dimensions

\begin{tabular}{|c|c|c|c|c|c|c|c|c|c|c|c|}
\hline & 1 & 2 & 3 & 4 & 5 & 6 & 7 & 8 & 9 & 10 & 11 \\
\hline 1. SEDS & 1.00 & .45 & .32 & .19 & .45 & .25 & .27 & .47 & .43 & .27 & .12 \\
\hline 2. SAPD & .45 & 1.00 & .48 & .35 & .41 & .47 & .58 & .54 & .49 & .40 & .03 \\
\hline 3. SCAD & .32 & .48 & 1.00 & .31 & .33 & .37 & .39 & .35 & .33 & .34 & -.09 \\
\hline 4. CMER & .19 & .35 & .31 & 1.00 & .21 & .28 & .53 & .26 & .32 & .34 & -.05 \\
\hline 5. FAES & .45 & .41 & .33 & .21 & 1.00 & 22 & .41 & .55 & .58 & .32 & .20 \\
\hline 6. $\mathrm{PDQF}$ & .25 & .47 & .37 & .28 & .22 & 1.00 & .51 & .33 & .36 & .39 & -.04 \\
\hline 7. SOCI & .27 & .58 & .39 & .53 & .41 & .51 & 1.00 & .45 & .51 & .50 & .01 \\
\hline 8. QPFS & .47 & .54 & .35 & .26 & .55 & .33 & .45 & 1.00 & .55 & .39 & .30 \\
\hline 9. $\mathrm{ORCH}$ & .43 & .49 & .33 & .32 & .58 & .36 & .51 & .55 & 1.00 & .41 & .12 \\
\hline 10. AACR & .27 & .40 & .34 & .34 & .32 & .39 & .50 & .39 & .41 & 1.00 & -.02 \\
\hline 11. AOFD & .12 & .03 & 年- 09 & -.05 & .20 & -.04 & .01 & .30 & .12 & -.02 & 1.00 \\
\hline
\end{tabular}




\section{One-Way Analysis of Variance}

To begin examining research question one, which asked to what extent is organizational effectiveness defined by environmental, social, and economic factors as they relate to public colleges and universities, a series of one-way analysis of variance (ANOVAs) were conducted for each institution. The purpose of conducting ANOVAs was to examine whether there are differences among constituencies within an institution in their ratings of effectiveness on each dimension, domain, as well as overall effectiveness. In order to further explore differences found among constituencies, follow-up tests using post hoc mean comparisons were conducted. Field (2009) notes that ANOVA is considered a reliable test under the normal distribution of the data. However, potential issues to consider relate to controlling the Type I error and the statistical power of the results (Type II error). Field reminds that there is a trade-off between controlling the Type I and Type II error and keeping Type I error at a conservative level can result in the loss of statistical power, which increases the possibility to miss a real difference in the data.

It is important to have these issues in mind when choosing post hoc procedures especially when group sizes are different as is the case in current research and when the group variances are very different. Given the assumption that group variances for some dimensions would be different, the decision was made to test for that as well. Thus, the post hoc procedure used to compare the constituencies were Levene's test to check for homogeneity of variance and Welch's test was used in cases when group variances were not equal. In addition, given different sample size among constituencies, Hochberg's GT2 
test was used when group variances were similar, and Games-Howell procedure was used in cases when group variances were different.

The results indicate that for Institution 1, there were significant differences among constituencies on the majority of dimensions, with the exception of the following three: Student Educational Satisfaction, Faculty, Administrators and Staff Employment Satisfaction, and Ability to Overcome Financial Difficulties (Table 8). Administrators, faculty, staff and students see Institution 1 as more effective in Student Academic and Personal Development than government and employers, $\mathrm{F}(5,697)=131.075, \mathrm{p}<.05$, while students, government and employers perceive Student Career Development as more effective than faculty and staff, $\mathrm{F}(5,659)=11.557, \mathrm{p}<.05$. Capacity to Maintain Resource Base was perceived as more effective by administrators, faculty and staff than by students, government and employers, $\mathrm{F}(5,680)=134.460, \mathrm{p}<.05$. Faculty evaluated Professional Development and Quality of the Faculty higher than administrators, F(2, $315)=5.547, \mathrm{p}<.05$, and this was the only dimension where internal constituencies differed in their ratings. System Openness and Interaction was assessed as more effective by administrators, faculty, staff, government and employers than by students $F(5,697)=$ 518.767, $\mathrm{p}<.05$ and administrators, faculty and staff perceived Resources for Quality Programs, Faculty and Students to be more effective than what students, government and employers thought, $\mathrm{F}(5,693)=56.881, \mathrm{p}<.05$. Organizational Culture and Health appeared to be higher rated for administrators, faculty and staff than students, $\mathrm{F}(3,597)=$ 881.077, $\mathrm{p}<.05$, while Ability to Acquire, Save and Use Resources Effectively was 
perceived as more effective by administrators, faculty and staff than by government and employers, $\mathrm{F}(4,401)=132.309, \mathrm{p}<.05$.

Institution 2 showed significant differences on seven dimensions, leaving results for four dimensions non significant (Table 9). The four dimensions that did not have significantly different ratings among constituencies were: Student Educational Satisfaction, Faculty, Administrator and Staff Employment Satisfaction, Ability to Overcome Financial Difficulties, and Student Career Development. Student Academic and Personal Development was perceived as more effective by administrators, faculty, staff and students than by external constituencies (government and employers), F(4, 296) $=54.560, \mathrm{p}<.05$ and administrators, faculty and staff perceived Capacity to Maintain or Expand Resource Base more effective than students and externals, $F(4,290)=35.516$, p $<.05$. Faculty gave higher rating to Professional Development and Quality of the Faculty than staff, $\mathrm{F}(2,98)=4.849, \mathrm{p}<.05$, while administrators, faculty, staff and externals perceived System Openness and Interaction to be higher than what students perceived, $\mathrm{F}(4,295)=161.622, \mathrm{p}<.05$. Institution 2 was perceived more effective in Resources for Quality Programs, Faculty and Student by administrators, faculty and staff than by students and externals, $\mathrm{F}(4,293)=79.793, \mathrm{p}<.05$, more effective by administrators, faculty and staff then students in Organizational Culture and Health, $F(3,272)=271.656$, $\mathrm{p}<.05$, and more effective by administrators, faculty and staff than by externals in the Ability to Acquire, Save and Use Resources effectively, $\mathrm{F}(3,119)=59.804, \mathrm{p}<.05$.

To examine differences among constituencies by domain and by overall effectiveness, additional ANOVAs were conducted for each of the two institutions 
studied. Results for both institutions showed significant differences among constituencies for each domain as well as for overall effectiveness (Table $10 \& 11$ ). Institution 1 was perceived as more effective in the Environmental Systems domain by administrators, faculty and staff than by students, government and employers $(\mathrm{F}(5,699)=53.452, \mathrm{p}<$ .05). In addition, students perceived the institution to be more effective in this domain than employers. The results for this domain for Institution 2 were similar (administrators, faculty and staff had higher ratings than students and externals), with the exception that there was no difference between students and externals in this domain $(F(4,296)=$ $19.292, \mathrm{p}<.05)$.

The Social Systems domain was perceived higher by administrators, faculty and staff than by students in both institutions $(\mathrm{F}(3,603)=1720.848, \mathrm{p}<.05$ for Institution 1 and $F(3,273)=434.058, p<.05$ for Institution 2, while Economic Systems domain was perceived as more effective by administrators, faculty and staff than by students, government and employers (Institution 1) and administrators, faculty and staff than by students and externals (Institution 2), $\mathrm{F}(5,699)=370.012, \mathrm{p}<.05$ and $\mathrm{F}(4,294)=$ $207.966, p<.05$ respectively. Finally, in terms of overall institutional effectiveness both institutions were perceived as more effective by administrators, faculty and staff than by students, government and employers (Institution 1) and students and externals (Institution 2), $\mathrm{F}(5,701)=386.154, \mathrm{p}<.05$ and $\mathrm{F}(4,296)=121.018, \mathrm{p}<.05$ respectively. 


\section{TABLE 8}

Analysis of Variance of Effectiveness Dimensions by Constituency - Institution 1

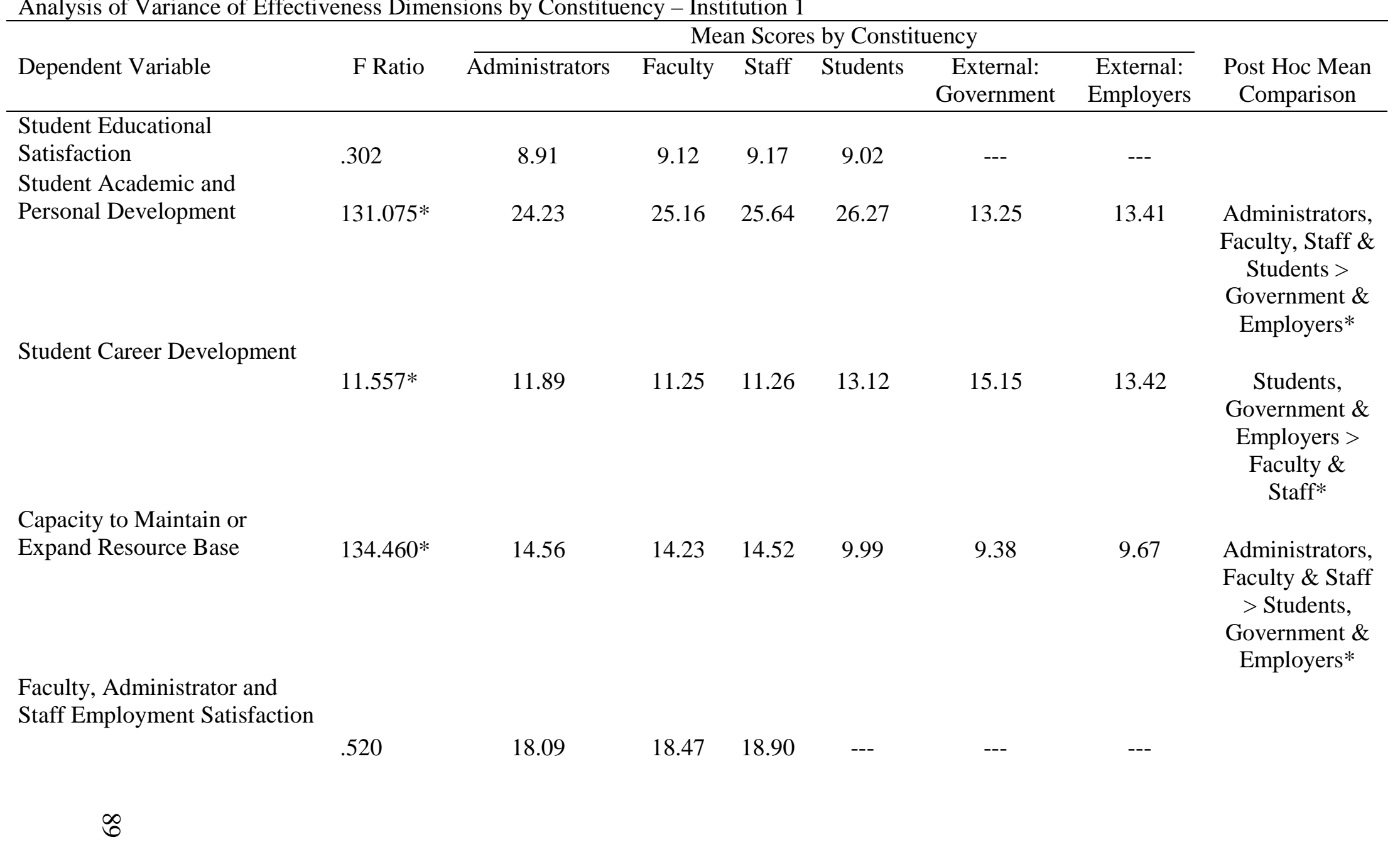


Professional Development and

Quality of the Faculty

$5.547 *$

11.39

12.93

11.97

$--$

$--$

Faculty >

System Openness and

Interaction

Administrators*

Resources for Quality

Programs, Faculty and

Students

$56.881 *$

12.82

13.18

14.13

9.53

9.41

Administrators,

Faculty \& Staff

$>$ Students,

Government \&

Employers*

Organizational Culture and

Health

881.077*

23.25

$23.71 \quad 22.83$

6.23

Ability to Acquire, Save and Use Resources Effectively

Faculty \& Staff

$>$ Government

\& Employers*

Ability to Overcome Financial Difficulties

3.19 


\section{TABLE 9}

Analysis of Variance of Effectiveness Dimensions by Constituency - Institution 2

\begin{tabular}{|c|c|c|c|c|c|c|c|}
\hline \multirow[b]{2}{*}{ Dependent Variable } & \multirow[b]{2}{*}{ F Ratio } & \multicolumn{5}{|c|}{ Mean Scores by Constituency } & \multirow[b]{2}{*}{$\begin{array}{l}\text { Post Hoc Mean } \\
\text { Comparison }\end{array}$} \\
\hline & & Administrators & Faculty & Staff & Students & $\begin{array}{c}\text { External: } \\
\text { Government } \\
\text { And Employers }\end{array}$ & \\
\hline Student Educational & & & & & & & \\
\hline $\begin{array}{l}\text { Satisfaction } \\
\text { Student Academic and }\end{array}$ & .549 & 11.04 & 10.45 & 11.00 & 10.61 & --- & \\
\hline Personal Development & $54.560^{*}$ & 26.88 & 27.07 & 27.92 & 28.54 & 13.09 & $\begin{array}{c}\text { Administrators, } \\
\text { Faculty, Staff } \\
\& \text { Students > } \\
\text { External* }\end{array}$ \\
\hline Student Career & & & & & & & \\
\hline $\begin{array}{l}\text { Development } \\
\text { Capacity to Maintain or }\end{array}$ & 1.955 & 14.62 & 14.30 & 14.96 & 13.31 & 14.00 & \\
\hline Expand Resource Base & $35.516^{*}$ & 14.64 & 14.32 & 15.17 & 10.86 & 10.36 & $\begin{array}{c}\text { Administrators, } \\
\text { Faculty \& Staff } \\
>\text { Students \& } \\
\text { External* }\end{array}$ \\
\hline $\begin{array}{l}\text { Faculty, Administrator and } \\
\text { Staff Employment }\end{array}$ & & & & & & & \\
\hline Satisfaction & .703 & 22.41 & 20.78 & 21.65 & --- & --- & \\
\hline $\begin{array}{l}\text { Professional Development } \\
\text { and Quality of the Faculty }\end{array}$ & $4.849^{*}$ & 14.78 & 15.56 & 12.74 & --- & --- & $\begin{array}{l}\text { Faculty }> \\
\text { Staff* }\end{array}$ \\
\hline
\end{tabular}


System Openness and Interaction

$161.622^{*}$

31.96

31.66

31.50

13.57

30.78

Administrators,

Faculty, Staff

$\&$ External >

Students*

Resources for Quality Programs, Faculty and Students

$79.793 *$

18.67

16.62

18.50

11.01

11.24

Administrators,

Faculty \& Staff

$>$ Students \&

External*

Organizational Culture and

Health

271.656*

24.86

26.81

24.68

6.99

$--$

Administrators,

Faculty \& Staff

$>$ Students*

Ability to Acquire, Save and Use Resources

$59.804^{*}$

11.48

12.23

12.58

$--$

6.70

Effectively

Ability to Overcome

Financial Difficulties

1.858

3.90

3.61

4.25

3.36
Administrators,

Faculty \& Staff

$>$ External*

$* p<.05$ 


\begin{tabular}{|c|c|c|c|c|c|c|c|c|}
\hline \multicolumn{9}{|l|}{ TABLE 10} \\
\hline \multicolumn{9}{|c|}{ Analysis of Variance of Effectiveness Domains and Overall Effectiveness by Constituency - Institution 1} \\
\hline \multirow[b]{2}{*}{ Dependent Variable } & \multirow[b]{2}{*}{ Ratio } & \multicolumn{6}{|c|}{ Mean Scores by Constituency } & \multirow[b]{2}{*}{$\begin{array}{l}\text { Post Hoc Mean } \\
\text { Comparison }\end{array}$} \\
\hline & & Administrators & Faculty & Staff & Students & $\begin{array}{c}\text { External: } \\
\text { Government }\end{array}$ & $\begin{array}{c}\text { External: } \\
\text { Employers }\end{array}$ & \\
\hline Environmental Systems & $53.452 *$ & 72.71 & 75.70 & 76.66 & 61.17 & 54.00 & 53.68 & $\begin{array}{c}\text { Administrators, } \\
\text { Faculty \& Staff > } \\
\text { Students, } \\
\text { Government \& } \\
\text { Employers* } \\
\text { Students > } \\
\text { Employers* }\end{array}$ \\
\hline Social Systems & $1720.848 *$ & 50.98 & 54.24 & 53.19 & 6.23 & --- & --- & $\begin{array}{l}\text { Administrators, } \\
\text { Faculty \& Staff }> \\
\text { Students* }\end{array}$ \\
\hline Economic Systems & $370.012 *$ & 38.93 & 41.94 & 42.03 & 19.34 & 26.88 & 28.03 & $\begin{array}{c}\text { Administrators, } \\
\text { Faculty \& Staff > } \\
\text { Students, } \\
\text { Government \& } \\
\text { Employers* } \\
\text { Government \& } \\
\text { Employers > } \\
\text { Students* }\end{array}$ \\
\hline \multicolumn{9}{|l|}{ Overall Institutional } \\
\hline Effectiveness & $386.154 *$ & 158.11 & 171.88 & 171.33 & 86.65 & 80.88 & 81.01 & $\begin{array}{c}\text { Administrators, } \\
\text { Faculty \& Staff > } \\
\text { Students, } \\
\text { Government \& } \\
\text { Employers* }\end{array}$ \\
\hline
\end{tabular}




\begin{tabular}{|c|c|c|c|c|c|c|c|}
\hline \multicolumn{8}{|l|}{ TABLE 11} \\
\hline \multicolumn{8}{|c|}{ Analysis of Variance of Effectiveness Domains and Overall Effectiveness by Constituency - Institution 2} \\
\hline \multirow[b]{2}{*}{ Dependent Variable } & \multirow[b]{2}{*}{ F Ratio } & \multicolumn{5}{|c|}{ Mean Scores by Constituency } & \multirow[b]{2}{*}{$\begin{array}{l}\text { Post Hoc Mean } \\
\text { Comparison }\end{array}$} \\
\hline & & Administrators & Faculty & Staff & Students & $\begin{array}{c}\text { External: } \\
\text { Government } \\
\text { And Employers }\end{array}$ & \\
\hline Environmental Systems & $19.292 *$ & 82.67 & 82.98 & 83.08 & 65.96 & 56.04 & $\begin{array}{c}\text { Administrators, } \\
\text { Faculty \& Staff } \\
>\text { Students \& } \\
\text { External* }\end{array}$ \\
\hline Social Systems & $434.058^{*}$ & 60.00 & 61.28 & 56.32 & 6.99 & --- & $\begin{array}{l}\text { Administrators, } \\
\text { Faculty \& Staff } \\
>\text { Students* }\end{array}$ \\
\hline Economic Systems & $207.966^{*}$ & 46.33 & 46.05 & 50.50 & 21.81 & 30.55 & $\begin{array}{c}\text { Administrators, } \\
\text { Faculty \& Staff } \\
>\text { Students \& } \\
\text { External* } \\
\text { External > } \\
\text { Students* }\end{array}$ \\
\hline $\begin{array}{l}\text { Overall Institutional } \\
\text { Effectiveness }\end{array}$ & $121.018^{*}$ & 186.50 & 190.31 & 187.88 & 94.76 & 85.26 & $\begin{array}{c}\text { Administrators, } \\
\text { Faculty \& Staff } \\
>\text { Students \& } \\
\text { External } *\end{array}$ \\
\hline
\end{tabular}

$* \mathrm{p}<.05$ 
Two-Way Analysis of Variance

The first step in exploring research question two, which asked to what degree resource dependence influences effectiveness of public colleges and universities, was to test whether there are institutional differences between institutions studied. Since previous tests determined significant differences among constituencies on most of the dimensions, all domains and overall effectiveness by institution, a series of factorial ANOVAs were conducted to determine the influence of institution and constituency as well as to test for possible interaction between the two on each dependent variable noted above. The results indicate a significant main effect for institution on all dependent variables, while constituency had significant main effect on all but four variables noted before. There was a significant interaction effect between institution and constituency on six out of fourteen dependent variables (Table 12-26).

Specifically, there was a significant main effect of institution on Student Educational Satisfaction $(\mathrm{F}(1,878)=69.372, \mathrm{p}=.000)$, Student Academic and Personal Development $(\mathrm{F}(1,994)=11.718, \mathrm{p}=.001)$, Student Career Development $(\mathrm{F}(1,946)=$ $39.239, \mathrm{p}=.000)$, Capacity to Maintain or Expand Resource Base $(\mathrm{F}(1,971)=5.429, \mathrm{p}=$ .020), Faculty, Administrator and Staff Employment Satisfaction $(F(1,421)=23.573, \mathrm{p}=$ $.000)$, Professional Development and Quality of the Faculty $(F(1,413)=28.258, p=$ $.000)$, System Openness and Interaction $(\mathrm{F}(1,993)=6.378, \mathrm{p}=.012)$, Resources for Quality Programs, Faculty and Students $(F(1,987)=161.596, p=.000)$, Organizational Culture and Health $(\mathrm{F}(1,869)=19.786, \mathrm{p}=.000)$, Ability to Acquire, Save and Use Resources Effectively $(\mathrm{F}(1,521)=10.308, \mathrm{p}=.001)$, Ability to Overcome Financial 
Difficulties $(\mathrm{F}(1,529)=4.223, \mathrm{p}=.040)$, Environmental Systems domain $(\mathrm{F}(1,996)=$ 23.097, $\mathrm{p}=.000)$, Social Systems domain $(\mathrm{F}(1,876)=34.971, \mathrm{p}=.000)$, Economic Systems domain $(\mathrm{F}(1,994)=69.484, \mathrm{p}=.000)$, and Overall Effectiveness $(\mathrm{F}(1,998)=$ $38.538, \mathrm{p}=.000)$.

Next, the main effect of constituency was significant on Student Academic and Personal Development $(F(4,994)=93.303, p=.000)$, Capacity to Maintain or Expand Resource Base $(\mathrm{F}(4,971)=151.775, \mathrm{p}=.000)$, Professional Development and Quality of the Faculty $(F(2,413)=8.425, \mathrm{p}=.000)$, System Openness and Interaction $(\mathrm{F}(4,993)=$ $559.612, \mathrm{p}=.000)$, Resources for Quality Programs, Faculty and Students $(\mathrm{F}(4,987)=$ 156.176, $\mathrm{p}=.000)$, Organizational Culture and Health $(\mathrm{F}(3,869)=1050.146, \mathrm{p}=.000)$, Ability to Acquire, Save and Use Resources Effectively $(F(3,521)=61.010, p=.000)$, Environmental Systems domain $(F(4,996)=71.382, \mathrm{p}=.000)$, Social Systems domain $(\mathrm{F}(3,876)=1892.318, \mathrm{p}=.000)$, Economic Systems domain $(\mathrm{F}(4,994)=554.867, \mathrm{p}=$ $.000)$, and Overall Effectiveness $(\mathrm{F}(4,998)=508.522, \mathrm{p}=.000)$.

Finally, there was a significant interaction effect between the institution and constituency on Student Career Development $(\mathrm{F}(4,946)=8.670, \mathrm{p}=.000)$, Resources for Quality Programs, Faculty and Students $(\mathrm{F}(4,987)=13.331, \mathrm{p}=.000)$, Organizational Culture and Health $(F(3,869)=3.032, \mathrm{p}=.029)$, Social Systems domain $(F(3,876)=$ $7.657, \mathrm{p}=.000)$, Economic Systems domain $(\mathrm{F}(4,994)=4.645, \mathrm{p}=.001)$, and Overall Effectiveness $(\mathrm{F}(4,998)=3.127, \mathrm{p}=.014)$ 
TABLE 12

Analysis of Variance for Student Educational Satisfaction Dimension by Institution and Constituency

\begin{tabular}{lcccc} 
Factor & $d f$ & $\mathrm{~F}$ & $\eta$ & $\mathrm{p}$ \\
\hline Institution & 1 & 69.372 & .073 & .000 \\
Constituency & 3 & .460 & .002 & .711 \\
Institution X Constituency & 3 & .634 & .002 & .593 \\
\hline $\mathrm{p}<.05$ & & & &
\end{tabular}

\section{TABLE 13}

Analysis of Variance for Student Academic and Personal Development Dimension by Institution and Constituency

\begin{tabular}{lcccc}
\hline Factor & $d f$ & $\mathrm{~F}$ & $\eta$ & $\mathrm{p}$ \\
\hline Institution & 1 & 11.718 & .012 & .001 \\
Constituency & 4 & 93.303 & .273 & .000 \\
Institution X Constituency & 4 & .836 & .003 & .502 \\
& & & &
\end{tabular}

\section{TABLE 14}

Analysis of Variance for Student Career Development Dimension by Institution and Constituency

\begin{tabular}{lcccc}
\hline Factor & $d f$ & $\mathrm{~F}$ & $\eta$ & $\mathrm{p}$ \\
\hline & & & & \\
Institution & 1 & 39.239 & .040 & .000 \\
Constituency & 4 & 1.195 & .005 & .312 \\
Institution X Constituency & 4 & 8.670 & .035 & .000 \\
& & & &
\end{tabular}




\section{TABLE 15}

Analysis of Variance for Capacity to Maintain or Expand Resource Base Dimension by Institution and Constituency

\begin{tabular}{lcccc} 
Factor & $d f$ & $\mathrm{~F}$ & $\eta$ & $\mathrm{p}$ \\
\hline & & & & \\
Institution & 1 & 5.429 & .006 & .020 \\
Constituency & 4 & 151.775 & .385 & .000 \\
Institution X Constituency & 4 & 1.129 & .005 & .341 \\
& & & &
\end{tabular}

\section{TABLE 16}

Analysis of Variance for Faculty, Administrator and Staff Employment Satisfaction Dimension by Institution and Constituency

\begin{tabular}{lcccc}
\hline Factor & $d f$ & $\mathrm{~F}$ & $\eta$ & $\mathrm{p}$ \\
\hline & & & & \\
Institution & 1 & 23.573 & .053 & .000 \\
Constituency & 2 & .574 & .003 & .563 \\
Institution X Constituency & 2 & .892 & .004 & .411 \\
& & & &
\end{tabular}

\section{TABLE 17}

Analysis of Variance for Professional Development and Quality of the Faculty Dimension by Institution and Constituency

\begin{tabular}{lcccc} 
Factor & $d f$ & $\mathrm{~F}$ & $\eta$ & $\mathrm{p}$ \\
\hline & & & & \\
Institution & 1 & 28.258 & .064 & .000 \\
Constituency & 2 & 8.425 & .039 & .000 \\
Institution X Constituency & 2 & 2.843 & .014 & .059 \\
& & & &
\end{tabular}




\section{TABLE 18}

Analysis of Variance for System Openness and Interaction Dimension by Institution and Constituency

\begin{tabular}{lcccc} 
Factor & $d f$ & $\mathrm{~F}$ & $\eta$ & $\mathrm{p}$ \\
\hline & & & & \\
Institution & 1 & 6.378 & .006 & .012 \\
Constituency & 4 & 559.612 & .693 & .000 \\
Institution X Constituency & 4 & .683 & .003 & .604 \\
& & & & \\
\hline $\mathrm{p}<.05$ & & & \\
\hline
\end{tabular}

\section{TABLE 19}

Analysis of Variance for Resources for Quality Programs, Faculty and Students Dimension by Institution and Constituency

\begin{tabular}{lcccc}
\hline Factor & $d f$ & $\mathrm{~F}$ & $\eta$ & $\mathrm{p}$ \\
\hline & & & & \\
Institution & 1 & 161.596 & .141 & .000 \\
Constituency & 4 & 156.176 & .388 & .000 \\
Institution X Constituency & 4 & 13.331 & .051 & .000 \\
& & & & \\
\hline $\mathrm{p}<.05$ & & &
\end{tabular}

\section{TABLE 20}

Analysis of Variance for Organizational Culture and Health Dimension by Institution and Constituency

\begin{tabular}{lcccc}
\hline Factor & df & $\mathrm{F}$ & $\eta$ & $\mathrm{p}$ \\
\hline & & & & \\
Institution & 1 & 19.786 & .022 & .000 \\
Constituency & 3 & 1050.146 & .784 & .000 \\
Institution X Constituency & 3 & 3.032 & .010 & .029 \\
& & & & \\
\hline $\mathrm{p}<.05$ & & &
\end{tabular}




\section{TABLE 21}

Analysis of Variance for Ability to Acquire, Save and Use Resources Effectively Dimension by Institution and Constituency

\begin{tabular}{lcccc} 
Factor & $d f$ & $\mathrm{~F}$ & $\eta$ & $\mathrm{p}$ \\
\hline & & & & \\
Institution & 1 & 10.308 & .019 & .001 \\
Constituency & 3 & 61.010 & .260 & .000 \\
Institution X Constituency & 3 & 1.314 & .008 & .269 \\
& & & & \\
\hline $\mathrm{p}<.05$ & & & \\
\hline
\end{tabular}

\section{TABLE 22}

Analysis of Variance for Ability to Overcome Financial Difficulties Dimension by Institution and Constituency

\begin{tabular}{lcccc}
\hline Factor & df & $\mathrm{F}$ & $\eta$ & $\mathrm{p}$ \\
\hline Institution & & & & \\
Constituency & 1 & 4.223 & .008 & .040 \\
Institution X Constituency & 3 & 1.139 & .006 & .333 \\
& 3 & 2.375 & .013 & .069 \\
\hline $\mathrm{p}<.05$ & & & & \\
\hline
\end{tabular}

\section{TABLE 23}

Analysis of Variance for Environmental Systems Domain by Institution and Constituency

\begin{tabular}{lcccc} 
Factor & $d f$ & $\mathrm{~F}$ & $\eta$ & $\mathrm{p}$ \\
\hline & & & & \\
Institution & 1 & 23.097 & .023 & .000 \\
Constituency & 4 & 71.382 & .023 & .000 \\
Institution X Constituency & 4 & .877 & .004 & .477 \\
& & & & \\
\hline $\mathrm{p}<.05$ & & &
\end{tabular}




\section{TABLE 24}

Analysis of Variance for Social Systems Domain by Institution and Constituency

\begin{tabular}{lcccc} 
Factor & $d f$ & $\mathrm{~F}$ & $\eta$ & $\mathrm{p}$ \\
\hline & & & & \\
Institution & 1 & 34.971 & .038 & .000 \\
Constituency & 3 & 1892.318 & .866 & .000 \\
Institution X Constituency & 3 & 7.657 & .026 & .000 \\
& & & & \\
\hline $\mathrm{p}<.05$ & & &
\end{tabular}

\section{TABLE 25}

Analysis of Variance for Economic Systems Domain by Institution and Constituency

\begin{tabular}{lcccc} 
Factor & $d f$ & $\mathrm{~F}$ & $\eta$ & $\mathrm{p}$ \\
\hline & & & & \\
Institution & 1 & 69.484 & .065 & .000 \\
Constituency & 4 & 554.867 & .691 & .000 \\
Institution X Constituency & 4 & 4.645 & .018 & .001 \\
& & & & \\
\hline $\mathrm{p}<.05$ & & & & \\
\hline
\end{tabular}

\section{TABLE 26}

Analysis of Variance for Overall Institutional Effectiveness by Institution and Constituency

\begin{tabular}{lcccc}
\hline Factor & df & $\mathrm{F}$ & $\eta$ & $\mathrm{p}$ \\
\hline & & & & \\
Institution & 1 & 38.538 & .037 & .000 \\
Constituency & 4 & 508.522 & .671 & .000 \\
Institution X Constituency & 4 & 3.127 & .012 & .014 \\
& & & & \\
\hline $\mathrm{p}<.05$ & & &
\end{tabular}


Funding Sources and Levels of Funding

The second step in exploring the degree to which resource dependence influences effectiveness of public colleges and universities (research question two), was to test whether there is a difference in means between the institutions based on sources and levels of funding. To that end, a series of two-tailed independent t-tests were performed for each effectiveness dimension, each domain and overall effectiveness. Funding levels were examined by source for each institution with the goal to determine existence of any patterns and relationships between funding and mean scores of effectiveness.

The examination determined that Institution 1 had a higher level of funding for state and public financial aid funding, while Institution 2 had a higher funding level for federal funding and higher spending per student FTE. Further examination revealed that if funding sources were combined based on levels of funding there might be a relationship between funding by institution and mean scores of effectiveness. Thus, the decision was made to compare the means between the Institution 1 with higher state and public financial aid funding combined and Institution 2 with higher federal funding and higher spending per student (Table 27). Lavene's Test of Equality of Variance was run to check the assumption of equal variance for each of the dependent variable. The tests showed three dependent variables not having homogenous variance and the test results represented below reflect this finding.

The t-tests showed that the institution with higher federal funding and spending per student FTE had higher means for nine out of eleven individual effectiveness dimensions, with seven being significantly higher: Student Educational Satisfaction 
$(\mathrm{t}(962)=-9.81, \mathrm{p}=.000)$, Student Academic and Personal Development $(\mathrm{t}(1101)=5.40$ $\mathrm{p}=.000)$, Student Career Development $(\mathrm{t}(996)=-5.95, \mathrm{p}=.000)$, Faculty, Administrator and Staff Employment Satisfaction $(\mathrm{t}(482)=-4.80, \mathrm{p}=.000)$, Professional Development and Quality of the Faculty $(\mathrm{t}(473)=-5.73, \mathrm{p}=.000)$, Resources for Quality Programs, Faculty and Students $(\mathrm{t}(541.492)=-7.69, \mathrm{p}=.000)$, and Ability to Acquire, Save and Use Resources Effectively $(\mathrm{t}(203.956)=-2.00, \mathrm{p}=.05)$. The institution with higher state and public financial aid funding had higher means for two dimensions, System Openness and Interaction and Organizational Culture and Health with only System Openness and Interaction being significantly higher $(\mathrm{t}(1101)=3.062, \mathrm{p}=.002)$. The $\mathrm{t}$-tests for effectiveness domains and overall institutional effectiveness showed higher means for Institution 2 on Environmental Systems and Economic Systems domain, and Overall effectiveness, while Institution 1 had higher mean for Social Systems domain. However, only Environmental Systems domain had a significantly higher mean $(\mathrm{t}(1106)=-3.37, \mathrm{p}$ $=.001)$, while there was no significant difference between the institutions for two other domains and overall institutional effectiveness. 


\section{TABLE 27}

Effectiveness Means by Institution Based on Funding Source and Level

\begin{tabular}{|c|c|c|c|c|c|}
\hline \multirow[t]{2}{*}{ Dimension/Domain/Overall } & \multicolumn{2}{|c|}{$\begin{array}{c}\text { Institution } 1 \\
\text { Higher State \& } \\
\text { Public Financial } \\
\text { Aid Funding }\end{array}$} & \multicolumn{2}{|c|}{$\begin{array}{c}\text { Institution } 2 \\
\text { Higher Federal } \\
\text { Funding \& Spending } \\
\text { per Student FTE }\end{array}$} & \multirow[b]{2}{*}{$t$} \\
\hline & $\mathrm{M}$ & SD & $\mathrm{M}$ & SD & \\
\hline $\begin{array}{l}\text { Student Educational Satisfaction } \\
\text { Student Academic and Personal }\end{array}$ & 9.03 & 2.221 & 10.53 & 2.212 & $-9.808^{*}$ \\
\hline Development & 23.72 & 7.246 & 26.34 & 7.742 & $-5.397^{*}$ \\
\hline $\begin{array}{l}\text { Student Career Development } \\
\text { Maintain or Expand Resource }\end{array}$ & 12.33 & 3.519 & 13.78 & 3.625 & $-5.950 *$ \\
\hline $\begin{array}{l}\text { Base } \\
\text { Faculty, Administrator and Staff }\end{array}$ & 12.09 & 3.217 & 12.22 & 3.061 & -.642 \\
\hline $\begin{array}{l}\text { Employment Satisfaction } \\
\text { Professional Development and }\end{array}$ & 18.48 & 5.036 & 21.08 & 5.575 & $-4.800 *$ \\
\hline $\begin{array}{l}\text { Quality of the Faculty } \\
\text { System Openness and }\end{array}$ & 12.28 & 3.482 & 14.42 & 3.819 & $-5.727 *$ \\
\hline Interaction & 23.08 & 10.274 & 21.00 & 10.507 & $3.062^{*}$ \\
\hline $\begin{array}{l}\text { Resources for Quality Programs, } \\
\text { Faculty and Students } \\
\text { Organizational Culture and }\end{array}$ & 11.40 & 3.545 & 13.44 & 4.258 & $-7.685^{*}$ \\
\hline $\begin{array}{l}\text { Health } \\
\text { Ability to Acquire, Save and }\end{array}$ & 15.60 & 9.658 & 14.72 & 10.315 & 1.244 \\
\hline $\begin{array}{l}\text { Use Resources Effectively } \\
\text { Ability to Overcome Financial }\end{array}$ & 9.95 & 3.314 & 10.72 & 4.207 & $-1.996^{*}$ \\
\hline Difficulties & 3.48 & 1.435 & 3.66 & 1.584 & -1.280 \\
\hline Environmental Systems Domain & 65.27 & 18.46 & 69.42 & 19.72 & $-3.365^{*}$ \\
\hline Social Systems Domain & 32.03 & 24.98 & 28.59 & 27.32 & 1.877 \\
\hline $\begin{array}{l}\text { Economic Systems Domain } \\
\text { Overall Institutional }\end{array}$ & 30.18 & 13.03 & 30.73 & 14.01 & -.632 \\
\hline Effectiveness & 122.07 & 52.52 & 125.91 & 55.77 & -1.099 \\
\hline
\end{tabular}




\section{Responses to Open-Ended Question}

The survey included an open-ended question asking respondent to share any comments regarding their perception of the effectiveness of the institution with which they are affiliated. This question was included to give respondents an opportunity to provide any additional input in areas they considered important. A total of 300 comments were provided, which accounted for $26.8 \%$ of total responses. Overall, comments were useful in expanding on the quantitative portion of the data. For example, some comments talked about the characteristics of the student body: "My ranking of how much extra academic work our students do reflects the fact that many of them are employed outside of school -- not that they are not intellectually curious or that our instruction does not inspire them to think beyond the course content." While the characteristics of the student body as a factor in effectiveness have not been explored in detail in this study, they might play a significant role and warrant further exploration.

Some comments contained suggestions or clarified responses: "The quality of this institution has increased dramatically over the past decade, but it is still viewed as second tier when compared to others in the state. A greater effort should be made to focus on research in science and technology and streamline administrative rivalries, which I understand can inhibit academic growth at this institution. Finally, some emphasis should be placed on marquee institutions such as sports, research and assistantships to attract those who will be enthusiastic about their affiliation with the University."

Others criticized some or all aspects of the university. Of interest here are seemingly negative perceptions internal constituencies have of their institution. There 
were a number of comments made by internal groups at both institutions criticizing their institution, its processes and outcomes. The following comment represents such a view: "This institution is characterized by low levels of organizational support. It is a relatively hostile environment for faculty. We are teaching a huge number of students which limits our ability to be effective. The best students at the University are truly exceptional and a joy to work with and a great many students here do not have adequate skills to perform college-level work. There are relatively few resources available to enhance their skills, especially in terms of writing. As an FYI, I left the questions blank about staff and administrator perspectives because honestly I don't know. We have so little interaction, I would just be guessing. Thanks for doing the survey."

A number of comments noted the financial situation of the institution and challenges associated with it: "The institution is one of the most severely understaffed ones I have worked in at the public level. For each new initiative, it seems the University operates without the awareness of the need for "bodies" that it will actually take to accomplish this objective. Also salaries are among the lowest in the country for recruiting people externally to work here. It is a great place to work, however, on many levels, but I have seen a lot of unfortunate turnover here of excellent staff who leave to take on better (paid) opportunities with more sane workloads attached. This is a huge loss to a place as large as this because the need for talent and institutional memory here is critical to being efficient and productive in the long term."

In addition, some shortcomings of the survey instrument were noted: "My only connection with your institution is to place undergraduate students in internships. Some 
are excellent; some are amazingly unprepared for the workplace. I could not relate to most questions on this survey." Another commenter stated: "Some of these questions I feel would be better asked of faculty or alums. I am not particularly aware of success or failure rates of graduates." It should be noted that most of the comments about the instrument came from external constituencies regarding their inability to respond to questions. This suggests that additional work might need to be done if the instrument is used with this constituency in future studies or a different sample of participants should be chosen.

Appendix F contains a larger sample of comments representing topics discussed above. Overall, many of the comments express dissatisfaction and frustration employees and students have with the institution with which they are affiliated. While there are also some positive comments noted by each constituency, a cursory look suggests that external constituencies had a higher number of positive comments at least as they relate to the employees they hire among the graduates of the institution. However, this observation should be taken with caution given smaller overall number of comments submitted by external constituencies.

\section{Summary}

A number of statistical procedures were used to examine research questions and provide answers to the hypotheses posed in this study. To accomplish this, a series of one and two-way ANOVAs was conducted and a series of independent sample t-tests was performed. Of the four hypotheses tested in this study, one was supported, two received partial support, and one had a limited support. The results of the study suggest that 
environmental, social, and economic factors grouped in three domains are strong contributors to organizational effectiveness in higher education, thereby supporting hypothesis $1 \mathrm{~b}$. The results related to this hypothesis also contributed to support and revise the proposed overall research model. The overall research model is revised to account for the influence of the institution type and other factors in overall effectiveness.

The groups of constituencies involved in the study did have different perceptions of effectiveness but there was no difference in perceptions for each of the six individual constituency groups. Thus, this hypothesis (hypothesis 1a) was partially supported suggesting that these constituencies could be grouped in three groups instead. Similarly, dependence on public funding and its influence on effectiveness (hypothesis 2) appear to depend on the sources of funding. Higher dependence on state funding and public financial aid funding seem to have negative influence on a number of individual dimensions of effectiveness, while federal funding and spending per student FTE is linked to positive ratings on a number of these dimensions.

The notion that institutions would be more effective if they addressed each of the three effectiveness domains simultaneously rather than one at the time had limited support (hypothesis 1c). Based on the information available to test this proposition, it cannot be stated with confidence that this would be the case, thus producing inconclusive results for this hypothesis. The next chapter will discuss these findings in more detail and address the implications for theory and practice. In addition, the theoretical foundations on which these hypotheses and findings are based will be discussed and suggestions for further research will be noted. 


\section{CHAPTER V}

\section{DISCUSSION}

This research examined the influence of environmental, social, and economic factors on organizational effectiveness with an emphasis on differences among public university constituencies. The results were used to test the applicability of the sustainability framework in assessing organizational effectiveness in public higher education. In addition, the role of resources in institutional effectiveness was explored with the focus on dependence on public resources.

\section{Research Questions and Overall Model}

The purpose of this research was to propose a framework for defining and assessing effectiveness in higher education and examine the role that resources play in effectiveness. It has been proposed that the construct of effectiveness is central to sustaining successful operations of higher education institutions. The following research questions were used to investigate the tenets put forward in this study:

1. To what extent is organizational effectiveness defined by environmental, social, and economic factors as they relate to public colleges and universities?

2. To what degree does resource dependence influence effectiveness of public colleges and universities?

To examine these questions and hypotheses discussed below, this research used a model where eleven dependent variables, which demonstrate dimensions of effectiveness in 
higher education organizations, were examined first separately and then clustered in three domains: environmental, social, and economic. ${ }^{1}$

Hypothesis 1a, which stated that perceptions of institutional effectiveness will differ based on the constituency (faculty, staff, administrators, students, government officials, employers) was partially supported. Of the fifteen variables explored, four (Student Educational Satisfaction; Student Career Development; Faculty, Staff and Administrator Employment Satisfaction; Ability to Overcome Financial Difficulties) did not show significant differences among any of the constituencies. Thus, there were significant differences among constituencies on eleven variables (Student Academic and Personal Development; Capacity to Maintain or Expand Resource Base; Professional Development and Quality of the Faculty; System Openness and Interaction; Resources for Quality Programs, Faculty and Students; Organizational Culture and Health; Ability to Acquire, Save and Use Resources Effectively; Environmental Systems; Economic Systems; Social Systems; Overall Effectiveness).

However, a look at the individual constituencies reveals interesting findings. There were no differences among internal university constituencies (faculty, administrators and staff) except for one variable, Professional Development and the Quality of the Faculty dimension. In addition, there was no difference on any of the variables examined between two external constituencies, government officials and

\footnotetext{
${ }^{1}$ The environmental domain of the effectiveness framework refers to the entities, organizations, groups and individuals an organization interacts with and is affected by in some way. The social domain is defined as the internal dimension of an institution and refers to the components such as faculty, administrators and staff workplace satisfaction, morale, organizational culture and health of the institution. The economic domain refers to the ability of an institution to acquire resources it needs, but also the ability to save and use resources effectively.
} 
employers. The student constituency exhibited greatest variability. In some cases, students tended to group with internal constituencies, in others, with external constituencies, and sometimes their responses were separate.

These results indicate that there might be a utility in defining three groups of constituencies when assessing perceptions of effectiveness (employees, students and external groups), rather than break constituencies in six groups as it was done in this research. Cameron (1978) found no difference among university employees based on the job they performed. He included administrators and department heads in his study, but there is no indication that faculty who did not have administrative functions were included in that study. The current study included faculty and staff, which was not previously the case in the studies of effectiveness performed by Cameron and others using his model. Therefore it was not known whether these groups would differ from the administrator group. Even though this research suggests that the three employee groups for the most part do not differ, these findings may not be definite given small number of institutions involved.

The significance of including constituencies not previously included in the studies on effectiveness stems from the belief that each of these constituencies plays an important role and is relevant to public higher education organizations. Faculty is the largest internal group in colleges and universities and can have an effect on organizational performance through their teaching, research and community service. Similarly, smooth functioning of many facets of the university depends on staff employees. In addition, staff is often the first point of contact with students and public 
and as such can have an influence on multiple dimensions of effectiveness. Thus, this research points to the fact that faculty and staff combined represents the majority of the employees in higher education institutions and as such play a significant role in creating an effective organization.

The addition of students in this research recognizes their importance as a constituency, not only as one of the main reasons for universities' existence, but also notes their increased role in financing higher education institutions through student tuition and alumni contributions. The inclusion of external constituencies beyond members of the board of higher education, who had been included in prior studies, allowed an examination of these groups in relation to the university effectiveness. The results can give universities a valuable insight into how these groups view their organizations, as well as information about their misperceptions. The external constituencies can have a direct or indirect effect on an organization through the influence on the flow of resources or their role in policy decisions, affecting the work of an organization and possibly its effectiveness.

Hypothesis $1 \mathrm{~b}$ stated that environmental, social, and economic factors will be strong contributors to organizational effectiveness in higher education. These factors were grouped in three domains, environmental, social, and economic systems domains with the purpose to test applicability of the sustainability framework in assessing effectiveness. This hypothesis was supported indicating that all three domains when tested separately account for high percentage of overall effectiveness. More specifically, Environmental Systems domain shares $73.6 \%$ of the variability with overall 
effectiveness, while Social and Economic domains share $88.9 \%$ and $86.4 \%$ of the variability respectively. The percentages above were obtained by calculating correlation coefficients between overall effectiveness and each domain and squaring them. The results were significant at the .01 level. As Field (2009) notes, squaring correlation coefficients provides a "measure of the amount of variability in one variable that is shared by the other" (p. 179).

These percentages, however also indicate that there is an overlap among domains in their influence on overall effectiveness. In order to look into these relationships further, partial correlations were calculated with the goal to parse out the unique effect of each domain. Partial correlations were computed for each pair of domains while controlling for overall effectiveness and the resulting coefficients were then squared. The assumption was that there would be effects that can be accounted for by each domain, but there would also be effects that could not be accounted for by any of the domains. The results support this assumption showing that $80 \%$ to $93 \%$ of the overall effectiveness can be accounted for by the three domains with the remaining $7 \%$ to $20 \%$ left to be explained by other factors. In particular, after controlling for overall effectiveness, the results indicate that environmental and social domain share $80 \%$ of the variance, environmental and economic share $12 \%$ and economic and social domain share $1 \%$ of the variance.

Due to the small number of institutions involved in this study, it was not possible to calculate unique effects of each domain. Rather, the effect of domain pairs were calculated and summed up to obtain the overall effect. This may not be a perfect measure, given that some unique effects for each domain could be and probably are present and are 
not accounted for with this methodology. It is also possible that the overall effect is overstated for the same reason and further research should evaluate this in more detail. Nevertheless, the results suggest that environmental, social, and economic factors strongly contribute to overall effectiveness thereby supporting hypothesis $1 \mathrm{~b}$.

This model leaves about $7 \%$ to $20 \%$ of the variance in overall effectiveness unexplained. The results of the factorial ANOVAs reported in the previous section found significant differences between institutions due to institution type. This difference accounted for anywhere from less than $1 \%$ to $14 \%$ of the variance depending on whether ANOVAs were performed for dimensions, domains or overall effectiveness. The main variable used in examining the overall research model was Overall Effectiveness. The results indicate that the institution type accounted for about $4 \%$ of the variance in the overall effectiveness. While the two studied institutions are classified as institutions of different type, it should be noted that they both are research institutions, albeit with different levels of research activity. There is no indication that this research designation alone would override all other differences, but the expectation is that this variance might be larger among institutions with very different missions. It is, however, important for testing of the overall model that the analysis points to significant differences due to institution type and future research should further examine the magnitude of this influence.

The question of institution type and the relationship of institution type to effectiveness have been explored in some depth in the literature review section of the study. It has been noted that prior to Cameron's (1978) research, the research on 
effectiveness in higher education was relevant only to a limited number of institutions of higher education. More specifically, criteria used to assess effectiveness did not pertain to institutions that are for example, regional, that educate more of non-traditional student population or are more student access oriented in their mission. Cameron attempted to change that and this research takes this goal a step further. To that end, it is important to note some of the characteristics of the institutions involved in this study. This will be important to take into account in any future studies testing this framework.

Institution 1 is a large urban institution located in a big city. According to the Carnegie classification, it is a research university with high research activity. Its undergraduate programs are relatively balanced between arts and sciences and professional fields with at least half of the graduate degrees corresponding to undergraduate majors, while its graduate programs are classified as doctoral, STEM dominant. Institution 1 is also classified as primarily nonresidential, medium full-time four-year selective institution with high transfer-in population, which means that $60-79 \%$ of its undergraduate student population attends full-time and at least $20 \%$ of its undergraduates are transfer students.

Institution 2 is a large comprehensive institution located in a mid-sized city. Carnegie classification classifies it is a research university with very high research activity. It is considered the state's flagship institution. Its undergraduate programs are, to a large degree, arts and sciences with some professional programs and at least half of the graduate degrees offered correspond to undergraduate majors. The graduate programs are classified as comprehensive doctoral. Institution 2 is classified as primarily 
nonresidential, full-time four-year selective institution with high transfer-in population which means that at least $80 \%$ of its undergraduate student population attends full-time and at least $20 \%$ of its undergraduates are transfer students.

Thus, in terms of comparison of the two institutions, it is important to note that these institutions have some similarities as well as some significant differences. The similarities come mainly from their research designation, in the sense that they are both research institutions, although the intensity of their research activity differs. Institution 1 has high research activity designation while Institution 2 is a very high research activity institution. In addition, both institutions serve a significant percentage of transfer students, although it should be pointed out that Institution 1 has a much higher percentage of transfer students within its undergraduate student population. Institution 1 has a large number of part-time students. About $35 \%$ of its undergraduates are part-time students as opposed to about $8 \%$ at Institution 2. Moreover, Institution 1 has a higher percentage of in state students, about $2 / 3$ of its undergraduates come from the home state while only a little over half of Institution's 2 undergraduates come from the state the institution is located in.

All these differences can have an influence on some aspects of effectiveness. As the study findings suggest, the differences related to institution type account for $1-14 \%$ of effectiveness. This variation comes, in part, due to the differences in some of the characteristics of each institution. It is clear from the information noted above that, for example, there are significant differences in the student population two institutions serve. This can have an effect not only on the academic preparation of the students they serve, 
but also on the ability of the students to finance their education, as well as on the overall student educational satisfaction.

Hypothesis 1c, which states that overall institutional effectiveness, and thus sustainability will be higher for institutions that address environmental, social, and economic factors simultaneously than for institutions focusing on only one of these factors at a time, had limited support mainly due to the number of cases involved in this study. While it was suggested that environmental, social, and economic factors, as defined in three domains, contribute significantly to overall effectiveness, this study is not conclusive about the effect of institutions simultaneously addressing all three domains. However, by looking at the results available from this study, including the contributions of the three domains and the role of constituencies in assessing effectiveness, there are indications that hypothesis 1c could be supported.

Moreover, starting from the proposition that all dimensions of effectiveness and consequently all three domains are of relatively equal importance for an institution, it follows that addressing the factors that influence all three domains would be beneficial for the institution. That said, there may be instances where an institution would want to focus on one domain more than on others for a period of time. This can be a result of new strategic initiatives, less than desirable level of effectiveness in a particular domain or other factors. As a whole, however, the expectation is that if an institution aspires to be effective, it would need to pay attention to all three domains simultaneously. Future studies involving a larger number of institutions should provide more conclusive answers to this question. 
Figure 3 below represents the revised theoretical model reflecting the findings of

this study.

Figure 3: Revised theoretical model of effectiveness in higher education

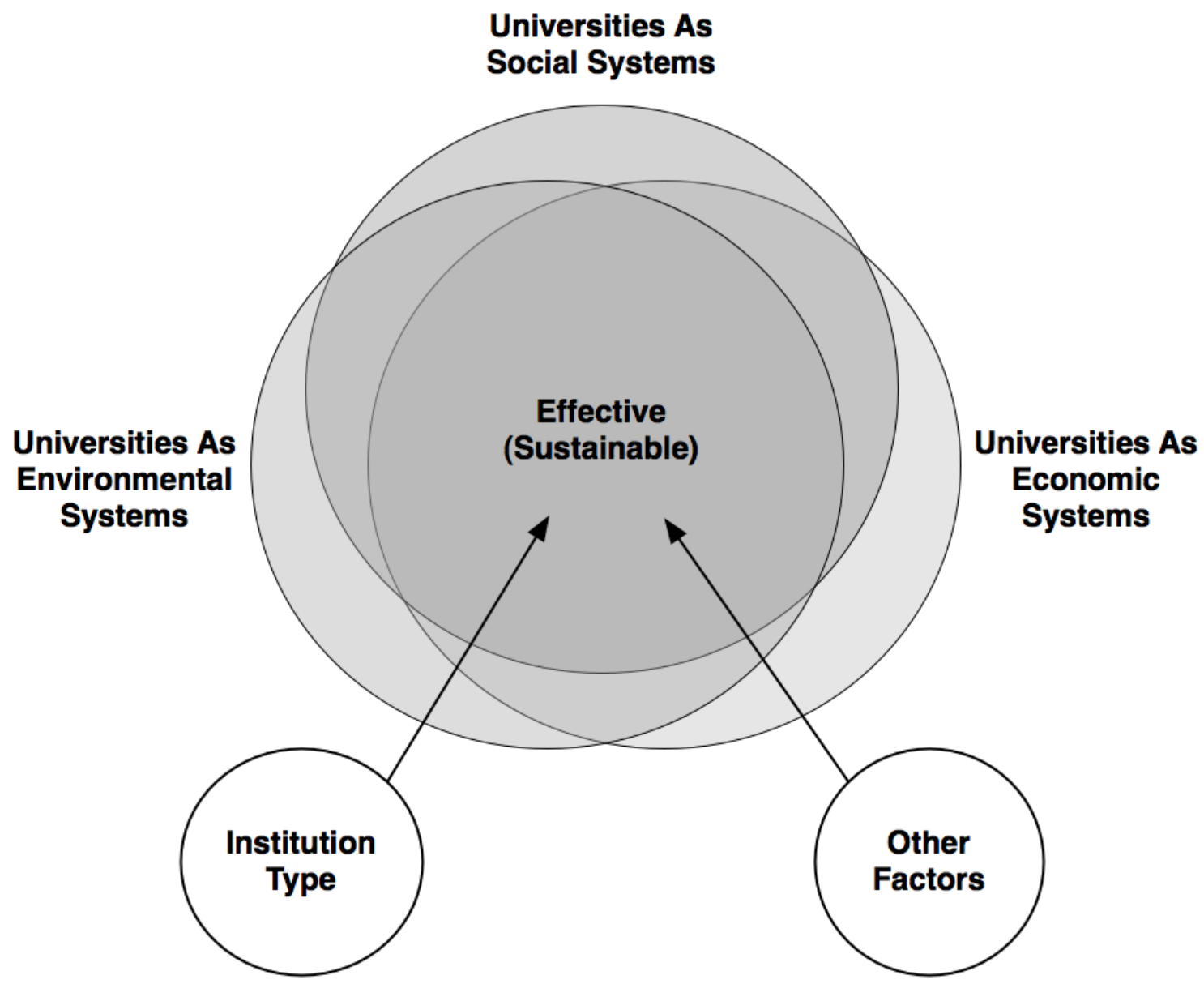

Note: The environmental systems domain of the effectiveness framework refers to the entities, organizations, groups and individuals an organization interacts with and is affected by in some way. The social systems domain is defined as the internal dimension of an institution and refers to the components such as faculty, administrators and staff workplace satisfaction, morale, organizational culture and health of the institution. The economic systems domain refers to the ability of an institution to acquire resources it needs, but also the ability to save and use resources effectively.

In testing hypothesis 2 , which postulated that the influence of resource

dependence on institutional effectiveness will differ depending on the predominant 
sources of public funding, three sources of public funding were taken into consideration: state funding, federal funding, and public financial aid funding. In addition, spending per student FTE was used as a fourth independent variable. This hypothesis had partial support indicating that dependence on state funding and public financial aid funding might have negative influence on some aspects of institutional effectiveness while dependence on federal funding and spending per student FTE has a positive effect. This was particularly true for all student outcomes dimensions (Student Educational Satisfaction, Student Academic and Personal Development, and Student Career Development), two of the four financial dimensions (Resources for Quality Programs, Faculty and Students and Ability to Acquire, Save and Use Resources Effectively) and two of the three internal dimensions (Faculty, Staff and Administrator Employment Satisfaction and Professional Development and Quality of the Faculty), for which Institution 2, with higher federal funding and spending per student, had significantly higher means. Institution 1, on the other hand, had significantly higher rating for the System Openness and Interaction dimension.

Institution 2 had a significantly higher mean difference on the Environmental Systems domain, while there was no significant difference between the institutions on Social and Economic Systems domains. In addition, there was no significant difference between the institutions in overall effectiveness. It might be of interest that the institutions did not differ in the economic domain despite the fact that their funding sources and levels of funding were different. However, one plausible explanation could be that they are located in the same state, indicating there are state factors other than 
funding that influence effectiveness. It is possible that comparing institutions from different states would yield different results and future research may wish to address that.

Overall, the findings suggest that higher dependence on state funding and public financial aid funding results in an institution being less effective on many of the dimensions. It might be beneficial for future studies to decouple these two sources of funding in order to parse out the effect of each separately. Results also suggest that this dependence may not be carried through to overall effectiveness. Thus, hypothesis 2 is supported with respect to individual dimensions but is not supported with respect to overall effectiveness.

While it was not examined in this study, the effectiveness of some of the individual dimensions could also be related to the student population institutions are serving. As noted before, Institution 1 has a large number of part-time students as well as adult students who have to balance academics with their work, family and other life demands. Furthermore, this institution as part of its mission admits a higher number of students who are not as academically prepared as students at Institution 2. It is possible that a combination of serving a student population requiring more institutional support with fewer resources than the institution has available to spend per student results in Institution 1 being perceived as less effective in student related dimensions. This proposition, if true, could explain some of the differences in student related dimensions between the institutions and future research may want to add student characteristics variables into the equation. 
Examination of hypotheses 1a, 1b, and 1c suggests that the first research question which asked about the extent to which organizational effectiveness is defined by environmental, social, and economic factors as they relate to public colleges and universities can, for the most part, be answered as to a large extent, with the caveat that the study should be repeated with a larger number of institutions and by contrasting institutions from different states. While it is not possible to generalize these findings to a population due to the small number of cases involved in this study, these findings provide support to the theoretical proposal regarding the application of the sustainability framework as a model of effectiveness and suggest that it could be used in assessing effectiveness in higher education institutions.

The answer to the second research question which asked: To what degree does resource dependence influence effectiveness of public colleges and universities, appears to depend on which sources of public funding an institution relies the most. To obtain a more robust answer to this question, it would be beneficial to look at the specific levels of funding by source and compare them among a larger number of institutions as well as look into the differences in student characteristics by institution.

\section{Effectiveness, Constituencies and Accountability}

In reviewing the objectives of this study, the attention returns to the construct of effectiveness, its theoretical basis, and the role of constituencies in assessing it. Past research (Cameron, multiple studies; Smart \& Hamm, 1993a; 1993b; Smart \& St. John, 1996), as well as this study, suggests that effectiveness is a multidimensional construct and effectiveness of individual dimensions within an organization can vary. This study 
identified eleven dimensions which were used to assess effectiveness in higher education. These dimensions were used in two ways: as individual dimensions, signifying specific measures within a particular dimension, and grouped in three domains with the purpose of building a theoretical framework that could then be applied for assessing effectiveness in colleges and universities.

An important point to note when discussing individual dimensions is the relationship between specific indicators within dimensions and overall effectiveness. As Ingraham (2005) notes, it is important that our performance metrics measure what is important. As institutions of higher education develop and refine their performance metrics, it is important to note that there are some traditional measures, which while important, do not capture everything higher education institutions are trying to accomplish.

The most common indicators of effectiveness in the ten states Ewell studied (as cited in Ruben, 1999) were: enrollment/graduation rates by gender, ethnicity, and program; degree completion and time to degree; persistence/retention rates by grade, ethnicity, and program; remediation activities and indicators of their effectiveness; transfer rates to and from two- and four-year institutions; pass rates on professional exams; job placement data on graduates and graduates' satisfaction with their jobs; and faculty workload and productivity in the form of student/faculty ratios and instructional contact hours (p. 3). As Ruben (1999) points out, "To some extent, as with business, higher education indicators have tended to be primarily historical, limited in predictive power, often incapable of alerting institutions to changes in time to respond, and have not 
given adequate consideration to important but difficult-to-quantify dimensions" (p. 3).

This study used many of Cameron's indicators, but to account for missing indicators and to re-conceptualize what constitutes effectiveness in higher education, additional indicators were developed for this research.

The role of constituencies in assessing organizational effectiveness, another objective of this study, has been a point of interest for other researchers as well (Connolly, Conlon \& Deutsch, 1980; Cameron, 1982; Tsui, 1990). The findings of this research indicate that there is a utility in asking multiple higher education constituencies their perceptions of effectiveness of a given institution. As noted before, there was a significant difference among groups of constituencies on the majority of dimensions. It was also discovered that some constituencies can be grouped based on the nature of their connection to the university. Thus, even though it was expected that internal constituencies (administrators, faculty and staff) will differ in their assessments, that expectation was not supported in this study. There was perhaps less surprise that two external constituencies, government and employers, did not differ in their ratings. The student constituency exhibited the greatest variability, sometimes siding with internal groups, other times with external and on occasions standing on their own. This suggests that there is enough variation in how students perceive the effectiveness of the university they attend that noting them as a separate group is warranted.

Cameron (1982) tested the multiple constituencies model empirically. He measured preferences of various constituencies, thus testing a conceptual model which states that organization is effective if it satisfies preferences of important constituencies. 
His results point out that there are differences among the constituencies he studied and that in most cases more effective organizations satisfy multiple constituencies. The constituencies included in his study were university administrators (executive, academic, financial, student affairs), trustees and representatives of major funders. He did not include faculty, staff or students. While there is no explanation about who the major funders were, there is also no indication that employers were involved in the study.

Tsui (1990) notes that most studies on constituencies begin with an assumption that "preference satisfaction is the major basis of the effectiveness judgment" (p. 480). This indeed is a theoretical concept Cameron tested. However, Tsui points out, that other factors can influence effectiveness judgment, without necessary satisfying preferences of the constituencies under study. One of these factors is cognitive processes involved in forming perceptions. By presenting certain information to constituencies, information they had not been aware of before, constituencies can change their evaluative judgment about an organization even though their preferences are not necessarily more satisfied. The second group of factors influencing effectiveness judgment involves social processes related to reputation. If an organization acquires reputation by, for example satisfying one constituency, and this constituency expresses favorable effectiveness judgment for that organization, this improved reputation over time can result in higher evaluative judgments by other constituencies even though they may not be any more satisfied than they had been before.

Tsui’s (1990) suggestions on constituencies' satisfaction can be useful in higher education. Affordability, value of a degree, and even value of higher education as a 
whole are some of the areas often criticized by policy makers and public. Having available effectiveness information and building on the idea that cognitive processes are important in how constituencies perceive effectiveness of organizations, colleges and universities have an opportunity to explain or dispute discrepancies between the reality and perceptions. Similarly, the question of reputation is particularly prevalent in higher education, especially with existence of national rankings, such as the U.S. World \& News Report and others. These ranking publications typically do not take into consideration all the comprehensive measures of institutional effectiveness. Nevertheless, they seem to support Tsui's notion that constituencies' evaluative judgments change through social processes created as a result of these rankings, even though their preferences may not necessary be satisfied.

This study did not empirically measure preferences of constituencies included in the study. Rather, it identified factors pertinent to various groups through the literature review as well as in conversations with relevant constituencies in the pilot phase of the survey. These activities led to a differentiation of the survey ultimately resulting in the three versions of the survey. For example, it was discovered that external groups did not have enough information or did not consider relevant some of the internal workings of an organization, such as employee satisfaction or professional development opportunities for faculty, administrators and staff. Similarly, students did not know or had preferences for some of the financial aspects of an organization. Thus to obtain useful information it was deemed appropriate to employ three versions of the survey. 
Connolly, Conlon \& Deutsch (1980) argue that organizational effectiveness can be viewed as a number of evaluative statements made by different constituencies, thus not treating effectiveness as a single statement. The current research supports this notion to a degree. It gives constituencies an important place in assessing effectiveness, but also sees a value in having combined statements of effectiveness for an organization. This view stems from the notion that effectiveness results can be used differently based on the needs of an organization and purpose for which evaluations will be used. This differentiation can be as simple as noting evaluations of overall effectiveness, either as a whole or by each constituency, to as complex as breaking down results to each individual effectiveness dimension or even going into each individual indicator or groups of indicators within dimensions.

Kells (1992) notes that performance indicators are "a factual or opinion information gathered from existing data bases or de novo, about the functioning of organizations or their constituent units and for various purposes (monitoring, decision support, comparing, evaluating, improving)" (p. 5). Sizer, Spee \& Borman (1992) note that performance indicators are most often used for monitoring, evaluation, dialogue, rationalization, and resource allocation - processes policy makers hope to lead to accountability. Layzell (1999) attempts to explain this intention by noting, "At the state level, accountability is operationalized through the setting of goals and objectives for higher education and the periodic measurement of progress toward those goals and objectives using accepted indicators" (p. 235). 
Besides the often touted use of effectiveness measures for external purposes of accountability, the internal purpose of improvement should not be ignored. However, as Borden (2010) notes, "Information gleaned from assessment for improvement does not aggregate well for public communication, and information gleaned from assessment for accountability does not disaggregate well to inform program-level evaluation". One of the reasons for this dichotomy is in what Shulock (2003) calls "culture gap", where the world of academia is fundamentally different from the world of policy making. Policy makers want summative, quick and concise measures that focus on relatively simplistic outcomes. Academia, on the other hand, finds, as Shulock (2003) points out, these measures inappropriate and even threatening. They lead to a concern "that such an approach can be punitive and can narrow society's concerns to those aspects of higher education that can be readily measured, at the expense of dearly held values" (p. 4).

This study with its focus on assessing the perceptions of various internal and external constituencies is also an attempt to narrow this culture gap. The dimensions of effectiveness put forward in this study are broad enough to respond to quick calls for specific outcomes, whether they come from policy makers, funding agents, students, parents or employers. At the same time, with the more specific indicators nested within each dimension, it is possible to identify areas of internal improvement and act on them as needed. Ewell (2009) suggests some strategies for balancing external calls for accountability with internal needs for improvement. Two of his strategies in particular might speak to the suggestions offered here: institutions should respond to the legitimate external concerns and institutions should strive for continuous improvement using 
evidence gathered through assessment activities. The availability of meaningful data measuring broad range of outcomes can guide improvement and satisfy calls for accountability accomplishing both of the objectives traditionally found in conflict.

This research relied on obtaining perceptive data about the institutions involved in the study. However, objective data is another important element of effectiveness assessment. Cameron (1978) attempted to include in his study objective data with mixed results. His main purpose of including objective data was to establish external validity for his nine dimensions of effectiveness. As he reported, some of the objective measures he needed were not available and some he deemed as not reliable as desired. There are valid reasons beyond establishing external validity to obtain objective measures of institutional effectiveness. They could be used as a comparison with perceptive data, giving institutions a way to assess similarities and differences between their actions and the perceptions people have of the same actions. In addition, objective data is necessary and, depending on the purpose, a required component for assessment. Thus, a right mix of objective and perceptual measures will give institutions a more comprehensive picture of their effectiveness.

\section{Effectiveness as Sustainability}

The findings of this study point that there is a value in applying the sustainability framework as a model of effectiveness in higher education. However, given an exploratory nature of this study, further research is needed in order to speak with confidence about the results. A few points should be noted in regards to decisions made 
in this study as they relate to the sustainability framework and its use as a model of effectiveness.

First, the use of the term and concept of environment is altered to mean environment in the organizational theory sense of the term. Even though the original meaning of environment as natural environment has been modified, it can be argued that its definition, given by Esty, Levy, Srebotnjak and de Sherbinin (2005), as a "long-term maintenance of valued environmental resources" (p. 11), generally applies in both cases. As Mellow (2008) states, "We must locate ourselves firmly in the context of a world that is radically different from the one that created the current systems of American colleges and universities. Without a more honest depiction, and absent an ability to accurately define, appropriately measure, and innovatively respond to reality, American higher education is not sustainable. Like an ecologically threatened environment, we must come to grips with what is undermining our ability to grow a sound ecosystem" (p. 29). Mellow goes on to pose a question about the elements and principles of a sound organizational ecosystem, suggesting some elements that in her view will help universities cope with current and future challenges. Similarly, the environmental domain in this study was used to address the organizational elements influencing universities from the external environment.

Second, social domain of the framework as put forward in this study refers only to internal components of higher education institutions to include professional development and the quality of the faculty, employee satisfaction, and organizational culture and health. It could be argued that in the sustainability framework, social domain goes 
beyond internal workings of a university and includes other social systems. While this argument is valid when looking at the sustainability framework in its original form, this research argues that in the adaptation of the framework as it was done in this study, it is more appropriate to place external elements of the system in other domains depending on their relationship to a university. That said, it is possible that there are components missing from the current model and future research should address that. Finally, the economic domain as proposed in this study underwent the least modification from its original meaning. Naturally, specific indicators are different and relevant to higher education institutions, but for the most part still refer to general economic principles.

This study aims to provide more understanding about the factors influencing effectiveness as well as measuring them. It was expected that examined institutions would be effective to a higher or lesser degree in each of the three domains (environmental, social, and economic), which was supported in the study. The expectation was also that the overall effectiveness and therefore an ability of institutions to sustain themselves in the long run would depend on how much universities attend to each of the three domains simultaneously. By looking at the results of the study, there are indications that this expectation could be supported as well, although more research is needed to be more conclusive about these results.

To support this latter hypothesis the notion of paradox, as advocated by some researchers (Cameron, 1986b, Weick 1976, Wagner, 2009), deserves to be further examined. According to Cameron (1986b), contradictory factors exist and operate at the same time in most organizations and the presence of this paradox, if addressed adequately 
enables organizations to adapt to turbulent conditions. In fact, in one of Cameron's studies on adaptation and effectiveness in higher education (1984), colleges and universities that were found successful in uncertain and turbulent environments were the ones that attended simultaneously to contradictions in their operations.

The concept of paradox has been linked to the concept of "janusian thinking". Rothenberg (1979) first introduced this term while doing research on creative people, researching, among others, Einstein and Picasso. The term originates from the Roman god Janus, who had many faces, with each face looking in opposite direction at the same time. According to Rothenberg, "Janusian thinking, consists of actively conceiving two or more opposite or antithetical ideas, images, or concepts simultaneously. Opposites or antitheses are conceived as existing side by side or as equally operative and equally true. Such thinking is highly complex" (p. 55).

Cameron (1984) notes, that this kind of complex thinking is necessary for flexibility of thought and problem solving, characteristics important for dealing with paradoxical organizations of today. Wagner (2009) points out that, "It has been said that paradox is like a gatekeeper to knowledge, denying passage to those who avoid it" (p. 4). Therefore, he suggests exploring paradox with all its facets and immersing into it in order to solve problems. Cameron notes not only the importance of janusian thinking as it relates to individuals in organizations, he also advocates for creation of organizations with janusian characteristics, which will enable them to better handle unpredictable events. The sustainability framework as a model of effectiveness, which advocates that its 
three domains are simultaneously attended to, may be well suited to help create organizations that deal successfully with the paradox.

\section{Resource Dependency Theory and Institutional Theory}

The conceptual base for the second research question was the influence of resource dependence on effectiveness with the particular focus on public resources. Public resources under consideration included state funding, federal funding and public financial aid funding. An additional independent variable included in testing of research question two was spending per student FTE. While further research is needed to achieve more robust results, the findings suggest that the Institution 1 which receives higher amount of state funding and higher amount of public financial aid is less effective in seven out of eleven dimensions of effectiveness.

In particular, dimensions associated with student educational satisfaction, student academic, personal, and career development, as well as some financial dimensions, were rated higher for the Institution 2 which receives more federal funding and spends more on students. On the other hand, Institution 1 appears to be significantly more effective in the System Openness and Interaction dimension. Additionally, it had higher rating in the Organizational Culture and Health dimension and Social Systems domain, although the difference was not significant. Thus, if institutions that receive more state funding and more public financial aid are less effective in the dimensions related to, for example, student outcomes, the question is why that would be the case and are there factors besides financial that have an influence on effectiveness. It also poses the question of whether 
there is a relationship between state funding and state control and what, if any, is its influence on effectiveness.

In looking at the theoretical concept of organizations' interactions with their environment, especially as it relates to resource dependence, literature review explored resource dependency theory and institutional theory. As a result, it was determined that there appears to be a value in using institutional theory to explain differences in effectiveness between two institutions. Resource dependency theory was deemed as not being able to fully explain environmental demands and organizational responses to these demands. It is important to note, however, that Pfeffer \& Salancik (2003), similarly to institutional theorists' view, also put an emphasis on the importance of the interaction with the environment and managing that environment. To that end they note, "Faced with conflicting demands, the organization must decide which groups to attend to and which to ignore" (p. 27).

While this is true, a difference between resource dependency theory and institutional theory lies in deciding how to respond to these demands. Pfeffer and Salancik would argue that when faced with conflicting demands, an organization makes decisions based on the level of resource dependence from each entity. They note, "To survive, organizations require resources. Typically, acquiring resources means the organization must interact with others who control those resources. In that sense, organizations depend on their environments" (p. 258). Institutional theory argues that organizations are often subject to isomorphic processes, which are according to DiMaggio and Powell (1983), constraining and force organizations to change and 
resemble other organizations that operate under similar environmental conditions. Thus, DiMaggio and Powell note, "individual efforts to deal rationally with uncertainty and constraint often lead, in the aggregate, to homogeneity in structure, culture, and output" (p. 147). This can also mean that isomorphic forces can prompt organizations to respond to environmental requests and influences other than financial, even though they depend on and need financial resources.

In looking at the results of this study, it appears that there are two sets of findings that need to be explained. One is the differences in effectiveness in individual dimensions. As noted before, the institution that receives more federal funding and spends more per student FTE was more effective on all student outcomes dimensions, two of the four financial dimensions and two of the three internal dimensions. Given that federal funding in this study, for the most part, relates to funding for grants and research, it could be proposed that a larger share of this type of funding will produce higher perceptions of effectiveness for some of the financial and internal dimensions.

While caution should be exercised when interpreting these results due to a small number of cases, some arguments can be put forward for further consideration. For example, Resources for Quality Programs, Faculty and Students, and Ability to Acquire, Save and Use Resources effectively are two financial dimensions shown to be significantly higher rated at Institution 2, which at least in part can be explained by higher research funding. The same may be true for Professional Development and the Quality of the Faculty and perhaps to a lesser extent for Faculty, Staff and Administrator Employment Satisfaction. However, there is no expectation that higher funding for 
research will result in a healthier and more supportive working environment. It could even be argued that high focus on research and a need to acquire more funding for research can work in an opposite direction. The results indicate that the Organizational Culture and Health dimension was rated lower at the Institution 2, although the difference was not significant.

It is, perhaps, noteworthy that effectiveness in the environmental domain, which includes dimensions such as student educational satisfaction, and student academic, personal, and career development, was significantly higher for the institution that receives more federal funding and spends more on student FTE. Research (Titus, 2006) does not indicate that more federal research funding results in better student outcomes at least as they relate to student persistence and graduation. However, Institution 2 also spends significantly higher amount per student than Institution 1. Even though research (Ehrenberg, 2007, Delta Cost Project, 2009) on the relationship between spending per student and quality of education is inconclusive mostly due to lack of developed quality measures, Blose, Porter and Kokkelenberg (2006) and Titus (2006) did find positive relationship between higher spending per student and graduation rates. While student outcomes such as the ones examined in this study cannot be equated with graduation rates, it is possible that there exists a positive relationship between the two. This would then explain higher effectiveness for the Institution 2 on these outcomes. It is, however, possible that higher ratings on student outcomes dimensions for Institution 2 could be related to factors other than the two financial indicators used in this study, particularly student characteristics factors. 
The second set of findings worth exploration relates to differences in overall effectiveness between the institutions. As noted before, even though Institution 2 appears to be more effective in seven out of eleven individual dimensions, this study did not find significant differences between institutions in overall effectiveness. Here is where the question of potential relationship between state funding and state control can be brought up again. Even though, Institution 2 receives a smaller portion of its overall funding from the state, it still is subject to state policies and control. Thus, it is possible that its relationship to the state influences its effectiveness. As Pfeffer and Salancik (2003) point out, "Part of the problem in understanding the environment as a concept is that the environment of an organization can affect an organization's outcomes without affecting its behavior. Important elements of the environment may be invisible to organizational decision makers, and hence, not considered in determining organizational actions, but these same elements can affect organizational success or failure" (p. 62-63).

One of the tenets of institutional theory is that even though an institution receives financial support from a group or entity, financial forces may not be the only ones through which that entity can affect the organization. In fact, other factors can and do influence institutions, sometimes even more than funding. The lack of difference in overall effectiveness found in this study serves to support this view as well as to support one of the theoretical premises of the study which stated that institutional theory better explains resource dependence in public higher education setting than resource dependency theory. In particular, it appears that Scott's (1995) concept of institutional 
pillars coupled with DiMaggio and Powell's notion of isomorphic changes are useful in explaining some of the findings of this study.

Scott (1995) identifies three elements or pillars of institutions - regulative, normative and cognitive. The regulative pillar refers to rules, laws and sanctions aimed to influence institutional behavior. The normative pillar includes norms and values and specifies how things should be done. The cognitive pillar refers to belief systems and symbolic aspects of social life in an organization. Similarly, DiMaggio and Powell's (1983) notion of isomorphism explains a change in an organization through coercive, mimetic and normative forces. Coercive isomorphism, as described by DiMaggio and Powell, "results from both formal and informal pressures exerted on organizations by other organizations upon which they are dependent and by cultural expectations in the society within which organizations function" (p. 150). Mimetic isomorphism derives from forces that encourage imitation and happens when organizations are faced with uncertainty. In these cases they model themselves after organizations they perceive as more successful. Lastly, normative isomorphism refers to pressures organizations and their members are subjected primarily through professionalization and are based either on formal education or on professional networks that span across organizations.

It is easy to see how higher education organizations with its complexities, multiple and ambiguous goals, and structures and culture resting on professions can be and are subject to all of these forces. And indeed, both Scott (1995), and DiMaggio and Powell (1983) note that organizations often exhibit all of these characteristics. In fact, DiMaggio and Powell note that the typology they developed is analytical and it is not 
always possible to distinguish it empirically. Nevertheless, they argue, "while the three types intermingle in empirical setting, they tend to derive from different conditions and may lead to different outcomes" (p. 150). Going back to the discussion related to this study, it is possible that a number of above mentioned institutional processes are underway in the two institutions studied. These processes cause them, partly by force through state regulations and partly through mimetic and normative pressures, to become similar in some of their characteristics resulting in the same overall effectiveness.

Higher federal funding and higher spending per student result in higher effectiveness in some university outcomes, but ultimately does not result in significantly higher overall effectiveness. To that end, it is worth noting that, "Although it is important to recognize that organizations may react to institutional pressures in a number of ways, it is also important to recognize the extent to which institutional environments influence and delimit what strategies organization can use" (Scott, 1995, p. 124). Thus, it is argued that the concepts within institutional theory offer a model to explain some of the behaviors seen in higher education institutions as well as provide some explanation for the findings of this study.

\section{Study Limitations}

In addition to the limitations of the study design noted in the methods chapter, there are some other limitations of this study. The first limitation pertains to the number of institutions involved in the study. While the number of participant responses was quite robust, it needs to be recognized that results are limited to just two institutions. Due to the exploratory nature of this study, it was deemed appropriate to examine the concepts 
studied and first attempt to generalize the findings to the theory (Yin, 2009). However, future studies involving more institutions would be needed in order to be able to generalize these results to the larger population of public higher education institutions. Another limitation was the fact that the sample population used in the research was restricted to one state. This was deemed an appropriate approach due to a desire to explore dependence on public funding within the same state context. But, in order to have generalizeable results, this research would need to be extended to other states.

It is important to note inconclusiveness of some of the findings. In regards to the influence of resource dependence on effectiveness, having a larger number of institutions would allow for different approaches to analysis, parsing out more details than was possible in this study. Similarly, while there are indications that the sustainability framework could be used as an appropriate theoretical model of effectiveness, a larger sample would be necessary to speak about it with confidence. Nevertheless, this is the first attempt to apply sustainability as theoretical framework in organizational effectiveness and as such is a valuable contribution to the field of organizational theory.

\section{Implications for Practice}

The results of this study yield several implications for practice. First, the difference among constituencies in their perceptions of effectiveness and the need for institutions to respond to the demands of these constituencies are important concepts to consider. Related to this is a need to have a comprehensive set of effectiveness indicators which encompass activities important for an institution to measure. As Ruben (2004) points out, it is important to develop a framework for measuring effectiveness and 
performance of higher education organizations by determining indicators that reflect the mission, aspirations and goals of the university. However, developing a satisfactory set of indicators is a challenge and Layzell (1999) and Nedwek (1996) note some limitations of existing indicator systems:

- Availability and limitations of data: sometimes data for adopted indicators were not previously collected, requiring an increase in time and cost necessary to start collecting missing data. Other times, in an attempt to avoid that problem, institutions adopt indicators for which data is already collected, thereby limiting the usefulness of the process.

- Number of indicators: there needs to be a balance between too many and too few indicators. The number of indicators often stems from a difference of opinion about what is important to measure. Thus, to address these differences, larger number of indicators is created making them potentially contradictory and less useful. On the other hand, too few indicators can present a problem as well by compressing several measures into smaller number of indicators and creating difficulties in measuring them.

- The relationship between policy and performance indicators: it is important to have a framework guiding policy decisions regarding performance indicators. The experience shows this is not always the case, which increases the possibility for collecting data not useful for decision-making.

- Quantitative vs. qualitative indicators: ideally, indicators will include both quantitative and qualitative measures. In reality, that is not often the case for a 
number of reasons, such as bias toward quantitative measures and difficulty in developing qualitative measures to address less tangible aspects of effectiveness.

- Inputs vs. processes vs. outcomes: It is often the case that inputs, processes and outcomes are mixed in developing indicators, without regard for the differences among them. Good indicators will address all three components but will also note differences among them.

- Use of indicator information: it is not always clear how the information collected for each indicator should be used. Ideally, the information should emphasize both accountability and institutional improvement. Focusing only on accountability lessens the use and acceptance of indicators.

- Lack of inclusion of relevant stakeholders in the creation of indicator: in some cases, indicators are developed without an input of broad base of stakeholders. While inclusive process takes longer and requires more effort, the indicators will ultimately be better if external and internal constituencies are involved from the beginning of the process.

The effectiveness dimensions developed in this research, along with specific items related to each dimension is an attempt to create a set of indicators that measure what is important while taking into consideration perspectives of a number of constituencies. Regardless of an attempt to be as inclusive as possible, it is possible and even expected that further use of these measures will identify missing indicators. Thus, refining the measures to fit institutional needs is a natural progression for practical use of effectiveness dimensions. As Ruben (2004) notes, "The usefulness of these indicators 
extends beyond performance measurement per se and contributes also to self-assessment, strategic planning, and the creation of focus and consensus on goals and directions within the organization" (pp. 97-98).

The finding that internal and external constituencies perceive effectiveness of an institution differently has at least two implications for university leaders. The first is a need to manage constituencies' expectations. At times this may involve simple tactics such as improved communications with relevant stakeholders or clarifying strategies an institution is pursuing. Other times it may lead to an attempt to modify constituencies' preferences or sometimes their perceptions. Tsui (1990) suggests analyzing cognitive and social processes leading to change in perceptions. For example, when looking at the reputation, her recommendation is to study how reputation is "created, how it persists, how it dissipates, and understanding how it may affect constituency expectations, perceptions, and judgment" (Tsui, 1990, p. 481). This can be an important further step in studying constituencies and their influence on institutions and does not have to be limited to reputation.

Sometimes, altering institutional practices to align it with constituencies' preferences is a viable option. Priorities may be reflected in how decisions are made and some decisions may result in the change of institutional priorities. It was interesting to note that there was no difference in perception in the study between internal and external constituencies regarding the extent to which institutions are open and interact with environment. The same is true for an ability of institutions to overcome financial challenges. Overall though, internal constituencies rated their institution higher on every 
domain and overall effectiveness. This suggests that government representatives and employers, which included businesses and non-profit organizations, view these institutions as less effective than internal groups. Thus, to the extent that funding and environment interact and to the extent that this interaction has an influence on an institution, whether purely in the financial sense or through policies and other means, these findings can have additional implications in how institutions decide to approach constituencies' perceptions.

Implications for Theory and Recommendations for Future Research This study addresses a gap in the research on organizational effectiveness in higher education. As the literature review revealed, there is no agreement on what constitutes effectiveness in higher education. Cameron's work and his nine dimensions of effectiveness represent an important contribution to this field of study. However, in his dimensions Cameron did not focus as much on external environment and its influence on effectiveness. Given the importance of external environment for higher education institutions, whether in regards to resources or other kinds of support or influence, it is essential to take this component into account when assessing effectiveness at the institutional level. This research refines Cameron's work by taking into consideration changes in higher education, including putting more emphasis on the external environment in which colleges and universities operate and the role resources play within it.

As a consequence of increased influence from the external environment as well as other changes in higher education, Cameron's nine dimensions of effectiveness were 
modified and expanded resulting in eleven dimensions developed in this study. Many of the changes can be attributed to the changes in the instrument, such as changes in wording of the questions or addition of new questions. Ultimately, one of Cameron's dimensions, Student Personal Development, did not load as a separate dimension during factor analysis and was combined with Student Academic Development on which it subsequently loaded. Some of the questions within Student Academic Development were dropped and substituted with what was deemed more relevant questions. In particular, questions related to academic rankings were dropped and new questions were added to reflect focus on student outcomes, such as level of knowledge and skills students develop during their academic career. Similarly, questions related to student career development are mostly substituted with new questions deemed as more appropriate indicators for measuring that dimension.

The addition of questions, particularly of a financial nature, resulted in three new dimensions added to the one already existing from Cameron's work. Questions added to the System Openness and Interaction dimension mostly loaded on that dimension during factor analysis. It should be noted, however, that some questions loaded relatively close on more than one dimension and because of the goals of this research were kept consistent with original Cameron's dimensions where possible. Other researchers (Smart \& Hamm, 1993a, 1993b) made similar decisions in the interest of comparing results with others who used the same model.

Thus, in terms of the quality of the instrument, this research attempted to update the instrument developed by Cameron. This work is not seen as final, however. Given 
that there may be indicators missing from the instrument, future research should focus on further refinement of the instrument. In addition, it would be beneficial to repeat factor analysis after additional items are included. To that end, it may be more appropriate to not be consistent with Cameron's dimensions, but rather strictly adopt the results of factor analysis.

This study uses the sustainability framework as a model of effectiveness and thus, not only addresses the lack of theoretical framework of effectiveness, but also expands the use of sustainability framework outside its common use. Initial results are optimistic, but additional research involving a larger number of institutions is needed in order to provide more conclusive recommendations for using this framework in higher education effectiveness assessment.

Moreover, the concept of environment is extended to mean environment in the organizational theory sense of the term. Scott (2003) suggests that organizations cannot be understood in isolation from their environment and if we are to be successful, we have to pay attention to all the actors associated with an organization. To that end, an investigation of internal and external constituencies aims to assess perceptions of these constituencies and possible implications for effectiveness.

One of the significances of the study is that it includes a number of constituencies not previously examined in the context of organizational effectiveness in higher education. As noted before, neither Cameron nor other researchers included faculty who did not perform administrative functions or staff in their research on effectiveness. This research changes that and includes both of these groups as they are considered crucial in 
maintaining successful operations of a university. Similarly, students and employers have not been included in other studies on effectiveness - this study examines their perceptions of effectiveness as well. While this research included a number of constituencies, it did not include all possible constituencies. Thus, further research on perceptions of the constituencies missing from this study, such as alumni and parents, might be beneficial and will add an important perspective of these groups.

This study uses perceptual data as primary means of collecting information about effectiveness. As such, it provides valuable insights about how some major constituencies see and assess the universities involved in the study. These findings can be a valuable asset to universities, especially to the extent that these constituencies can influence funding, policy decisions, or new student enrollment. However, it would be of great value to repeat this study using the same measures with objective data collected by institutions and compare findings. This can help open the door for the discussions about effectiveness, improvement and accountability and how they interrelate.

This study began to explore the theoretical under-pinnings of higher education organizations and their interactions with the environment with the particular attention to resource dependence aspect of this interaction. The resource dependency theory argues that organizations respond to the expectations of their funders (Pfeffer \& Salancik, 2003). Institutional theory, on the other hand, asserts that institutional complexities affect organizational structure and behavior and the resource dependence can be examined through the lenses of institutional theory (Scott, 1995). This study examines the role of resource dependence and its consequences for institutional effectiveness and argues that 
institutional theory can better explain this interaction with the environment than resource dependency theory. Continuing research to further explore this concept would be beneficial.

There are other questions related to effectiveness this study did not explore. Resource dependence was explored only as dependence on public resources. Given the proportion of funding that comes from other sources, it would be beneficial to explore how other funding sources influence effectiveness. Role and influence of constituencies is another area that deserves further consideration. How much influence should be allowed to particular constituencies and how much discretion universities can afford in this arena? This research compared effectiveness of one institution with another without having established a desired level of effectiveness either for individual dimensions or for overall effectiveness. Is there a value in establishing some benchmarks to which institutions would strive to or would that be just another way of competition among institutions? Finally, the notion of paradox and its relationship to effectiveness is mentioned in the discussion. If effectiveness is paradoxical requiring institutions to juggle multiple dimensions and find balance among them, the question becomes: Is there a value in having one overall effectiveness score and does it tell us anything useful about an institution?

Higher education institutions are under an increasing pressure to become more effective and more responsive to the demands of their constituencies. In order to respond, colleges and universities need to know how effective they are. The model of assessing effectiveness put forward in this research advances this task a step further. Having 
information about an institution's effectiveness can help universities not only become more accountable to their external constituencies but also start conversations with internal constituencies about needed improvements. To that end, the construct of effectiveness deserves to be continually explored in a search for better ways to measure it.

\section{Conclusion}

Today's colleges and universities are expected to be responsive to various societal demands, have a greater role in the economic development, and satisfy multiple constituencies especially to the degree that their funding base depends on the perceptions of these constituencies about their effectiveness.

Even though a need to assess organizational effectiveness is very much alive, research on how to assess it has not been robust in recent years. This study intends to help revive that research and enable higher education institutions to respond to challenges and make informed decisions regarding changes needed to ensure their sustainability as institutions contributing to the society in both public and private arena. And while the traditional measures of success, such as faculty reputation, quality of the student body and others discussed before, are important, they as Ruben (1999) notes "do not reflect some of the key success factors for a college or university, nor do they capture many of the dimensions of a university's mission, vision, or strategic direction" (p. 3).

This study furthers the task of identifying measures of success for a broader set of public colleges and universities. The findings suggest that organizational environment, social, and economic factors as defined in this study account for a large proportion of 
overall effectiveness and paying attention to all three simultaneously might be beneficial in building effective colleges and universities. In addition, the perceptions of students, internal, and external constituencies differ significantly from each other to warrant treating them as three separate groups in further explorations of the construct of effectiveness. Finally, the results indicate that the sources of public funding on which institutions depend most may influence effectiveness of many of the individual effectiveness dimensions proposed in this study. Thus, this study and its findings attempt to help institutions of higher education meet challenges they face while preserving their intrinsic values.

Zemsky and Wegner (1997) state that, "Higher education will either transform itself or be transformed as new markets, new technologies, and new competitors recast the business of the business - changing, in the process, how colleges and universities organize and deliver instructions as well as how they structure and manage their enterprises" (p. 72). For this transformation to happen successfully, colleges and universities will have to look both inward and outward when formulating strategies and making decisions. Developing a capacity to assess both internal and external indicators of excellence will enhance institutional ability to engage its constituencies and have more control over its existence. Zemsky and Wegner (1997) note,

"Once, it was possible for colleges and universities to look inward and define the challenges confronting them in their own terms; they could presume that the answers to problems in society could be found in the curriculum as they defined it. Much of the academy still wishes it enjoyed that sense of autonomy and deference once accorded institutions of learning. Indeed, the question most often asked within the academy is still, "How can society be made to recognize and support the value of what we do?" In contrast, the question now regularly asked 
by legislators, employers, parents, and students is, "How can higher education serve us better?" (p. 72).

This study suggests that both of these questions should be asked if higher education is to sustain itself and its role in the society. 


\section{REFERENCES}

Adams, W. M. (2006). The future of sustainability: Re-thinking environment and development in the twenty-first century. The World Conservation Union. Retrieved May 3, 2009 from http://cmsdata.iucn.org/downloads/iucn_future_of_sustanability.pdf.

Alexander, F. K. (2006). Concluding remarks. In R. G Ehrenberg (Ed.), What's happening to public higher education? American Council on Education: Praeger Series on Higher Education.

Andrews, R. (2008). An institutionalist approach to spatial variations in public service failure: Evidence from England. European Urban and Regional Studies, 15(4), 349362.

Babbie, E. R. (1973). Survey research methods. Belmont, CA: Wadsworth Publishing Company, Inc.

Beckhard, R. \& Harris, R. T. (1987). Organizational transitions: Managing complex change. Addison-Wesley Publishing Company.

Bergquist, W. H. \& Pawlak, K. (2008). Engaging the six cultures of the academy. San Francisco: Jossey-Bass Publisher.

Biemer, P. P. \& Lyberg, L. E. (2003). Introduction to survey quality. John Wiley \& Sons Publications.

Birnbaum, R. (1988). How colleges work: The cybernetics of academic organization and leadership. San Francisco: Jossey-Bass Publishers.

Blose, G. L., Porter, J. D. \& Kokkelenberg, E. C. (2006). The effect of institutional 
funding cuts on baccalaureate graduation rates in public higher education. In R. G. Ehrenberg (Ed.), What's happening to public higher education? American Council on Education: Praeger Series on Higher Education.

Borden, V. M. H. (2010, April 30). The accountability/improvement paradox. Inside Higher Ed. Retrieved March 15, 2011 from http://www.insidehighered.com/views/2010/04/30/borden.

Breneman, D. W. \& Finney, J. E. (1997). The changing landscape: Higher education finance in the 1990s. In P. M. Callan \& J. E. Finney (Eds.), Public and private financing of higher education: Shaping public policy for the future. American Council on Education: Oryx Press.

Brigham, S.E. (1996). Large scale events: New ways of working across the organization. Change: The Magazine of Higher Learning, 28 (6), 28-39.

Cameron, K. S. (1978). Measuring organizational effectiveness in institutions of higher education. Administrative Science Quarterly, 23, 604-632.

Cameron, K. S. (1980). Critical questions in assessing organizational effectiveness. Organizational Dynamics, 9(2), 66-80.

Cameron, K. S. (1981). Domains of organizational effectiveness in colleges and universities. The Academy of Management Journal, 24(1), 25-47.

Cameron, K. S. (1982). An empirical investigation of the multiple constituencies model of organizational effectiveness. National Center of Higher Education Management Systems. (ERIC Document Reproduction Service No. ED271069). 
Cameron, K. S. (1984). Organizational adaptation and higher education. The Journal of Higher Education, 55(2), 122-144.

Cameron, K. S. (1986a). A study of organizational effectiveness and its predictors. Management Science, 32(1), 87-112.

Cameron, K. S. (1986b). Effectiveness as paradox: Consensus and conflict in conceptions of organizational effectiveness. Management Science, 32(5), 539-553.

Cameron, K. S. (2005). Organizational effectiveness: Its demise and re-emergence through positive organizational scholarship. In K. G. Smith \& M. A. Hitt (Eds) Great minds in management: The process of theory development. Oxford University Press.

Cameron, K. S. \& Ettington, D. R. (1988). The conceptual foundations of organizational culture. In J. C. Smart (Ed.), Higher education: Handbook of theory and research, volume IV.

Cameron, K. S. \& Whetten, D. A. (1983). Some conclusions about organizational effectiveness. In K.S. Cameron \& D. A. Whetten (Eds) Organizational effectiveness: A comparison of multiple models. New York: Academic Press

Cameron, K. S. \& Whetten, D. A. (1996). Organizational effectiveness and quality: The second generation. Higher education: Handbook of theory and research, volume XI.

Clark, B.R. (1998). Creating entrepreneurial universities: Organizational pathways of transformation. New York: Pergamon Press.

Clott, C. B. (1995). The effects of environment, strategy, culture, and resource dependency on perceptions of organizational effectiveness of schools of business. ASHE annual meeting paper. (ERIC Document Reproduction Service No. 
ED391407).

Cohen, B. H. \& Lea, R. B. (2004). Essentials of statistics for the social and behavioral sciences. John Wiley \& Sons, Inc.

Cohen, J. (1988). Statistical power analysis for the behavioral sciences, $2^{\text {nd }}$ ed. Lawrence Erlbaum Associates, Publishers.

Cohen, J. (1992). Quantitative methods in psychology: A power primer. Psychological Bulletin 112(1), 155-159.

Cohen, J., Cohen, P., West, S. G., \& Aiken, L. S. (2003). Applied multiple regression/correlation analysis for the behavioral sciences, 3rd Ed. Mahwah, NJ: Lawrence Erlbaum Associates.

Connolly, T., Conlon, E. J. \& Deutsch, S. J. (1980). Organizational effectiveness: A multiple constituency approach. The Academy of Management Review, 5(2), 211-217.

Cooper, P. J. \& Vargas, C. M. (2004). Implementing sustainable development: From global policy to local action. Rowman \& Littlefield Publishers, Inc.

DiMaggio, P. J. \& Powell, W. W. (1983). The iron cage revisited: Institutional isomorphism and collective rationality in organizational fields. American Sociological Review, 48(2), 147-160.

Ehrenberg, R. G. (2006). The perfect storm and the privatization of public higher education. Change: The Magazine of Higher Learning, 38(1), 46-53.

Ehrenberg, R. G. (2007). The economics of tuition and fees in American higher education. Retrieved October 13, 2009, from Cornell University, ILR School site: 
http://digitalcommons.ilr.cornell.edu/cgi/viewcontent.cgi?article=1068\&context=working papers

Esty, D. C., Levy, M., Srebotnjak, T. \& de Sherbinin, A. (2005). 2005 Environmental Sustainability Index: Benchmarking National Environmental Stewardship. New Haven: Yale Center for Environmental Law \& Policy. Retrieved May 21, 2010 from http://www.yale.edu/esi/ESI2005_Main_Report.pdf.

Ewell, P. T. (2009). Assessment, accountability, and improvement: Revisiting the tension. Occasional Paper \#1. National Institute for Learning Outcomes Assessment.

Fairweather, J. S. \& Hodges, J. P. (2006). Higher education and the new economy. The Education Policy Center at Michigan State University.

Field, A. (2009). Discovering statistics using SPSS, $3^{\text {rd }}$ ed. Sage Publications Ltd.

Frye, R. (1999). Assessment, accountability, and student learning outcomes. Dialogue, 2.

Georgopoulos, B. S. \& Tannenbaum, A. S. (1957). A study of organizational effectiveness. American Sociological Review, 22(5), 534-540.

Gioia, D. A. \& Thomas, J. B. (1996). Identity, image, and issue interpretation: Sensemaking during strategic change in academia. Administrative Science Quarterly, 41(3), 370-403.

Gumport, P.J., \& Pusser, B. (1999). University restructuring: The role of economic and political contexts. Higher Education: Handbook of Theory and Research, 14, 146-200.

Gumport, P.J. (2000). Academic restructuring: Organizational change and institutional imperatives. Higher Education, 39, 67-91.

Guskin, A. E. (1996). Facing the future: The change process in restructuring universities. 
Change: The Magazine of Higher Learning, 28(4), 26-37.

Hossler, D. (2004). Refinancing public universities: Student enrollments, incentive-based budgeting, and incremental revenue. In E. P. St. John \& M. D. Parsons (Eds.), Public funding of higher education: Changing contexts and new rationales. Baltimore and London: The Johns Hopkins University Press.

Huisman, J. \& Currie, J. (2004). Accountability in higher education: Bridge over troubled water? Higher Education, 48(4), 529-551.

Ingraham, P. W. (2005). Performance: Promises to Keep and Miles to Go. Public Administration Review, 65(4), 390-395.

Katz. D. \& Kahn, R. L. (2005). Organizations and the system concept. In J. M. Shafritz, J. S. Ott, \& Y. S. Jang (Eds,), Classics of Organization Theory, 6th ed. New York: Wadsworth.

Kells, H. R. (1992). Performance indicators for higher education: A critical review with policy recommendations. The World Bank: PHREE Background Paper Series.

Layzell, D.T. (1999). Linking performance to funding outcomes at the state level for public institutions of higher education. Research in Higher Education, 40(2), 233-46.

Lewin, A. Y. \& Minton, J. W. (1986). Determining organizational effectiveness: Another look, and an agenda for research. Management Science, 32(5), 514-538.

Likert, R. (1967). The human organization: Its management and value. New York: McGraw-Hill.

Lysons, A. (1990). Dimensions and domains of organizational effectiveness in Australian 
higher education. Higher Education, 20, 287-300.

Lysons, A. \& Hatherly, D. (1992). Cameron's dimensions of effectiveness in higher education in the U.K.: A cross-cultural comparison. Higher Education, 23, 221-230.

Massy, W. F. (1996). Resource allocation in higher education. Ann Arbor: The University of Michigan Press.

Mellow, G. (2008). Creating a sustainable higher education system. The Presidency, $11(2), 28-33$.

Meyer, J. W. (1994). Rationalized environments. In W. R. Scott \& J. W. Meyer (Eds). Institutional environments and organizations: Structural complexity and individualism. Sage Publications.

Morgan, G. (1997). Images of organizations, $2^{\text {nd }}$ edition. Thousand Oaks, California: Sage Publications, Inc.

Nedwek, B. P. (1996). Public policy and public trust: The use and misuse of performance indicators in higher education. Higher education: Handbook of theory and research, volume XI.

Pascarella, E. T. \& Terenzini, P. T. (1991). How college affects students. San Francisco: Jossey-Bass.

Pfeffer, J. \& Salancik, G. R. (2003). The external control of organizations: A resource dependence perspective. Stanford, California: Stanford University Press.

Price, J. L. (1971). The study of organizational effectiveness. The Sociological Quarterly, 13(1), 3-15.

Quinn, R. E. \& Rohrbaugh, J. (1981). A competing values approach to organizational 
effectiveness. Public Productivity Review, 5(2), 122-140.

Roherty, B. M. (1997). The price of passive resistance in financing higher education. In P. M. Callan \& J. E. Finney (Eds.), Public and private financing of higher education: Shaping public policy for the future. American Council on Education: Oryx Press.

Romzek, B. S. (2000). Dynamics of public sector accountability in an era of reform. International Review of Administrative Science, 66, 21-44.

Rothenberg, A. (1979). The emerging goddess: The creative process in art, science, and other fields. The University of Chicago Press.

Ruben, B. D. (1999). Toward a balanced scorecard for higher education: Rethinking the college and university excellence indicators framework. White Paper, QCI, Center for Organizational Development and Leadership, Rutgers University.

Ruben, B. D. (2004). Pursuing excellence in higher education: Eight fundamental challenges. San Francisco: Jossey-Bass.

Schmitz, C. C. (1993). Assessing the validity of higher education indicators. The Journal of Higher Education, 64(5), 503-521.

Schneider, B., Parkington, J. J. \& and Buxton, V. M. (1980). Employee and customer perceptions of service in banks. Administrative Science Quarterly, 25(2), 252-267. Scott, W. R. (1995). Institutions and organizations. Thousand Oaks, London: Sage Publications.

Scott, W. R. (2003). Organizations: Rational, natural and open systems. New Jersey: Prentice Hall.

Scott, W. R. \& Meyer, J. W., eds. (1994). Institutional environments and organizations: 
Structural complexity and individualism. Sage Publications.

Seashore, S. E. \& Yuchtman, E. (1967). Factorial analysis of organizational performance. Administrative Science Quarterly, 12(3), 377-395.

Shulock, N. (2003). A fundamentally new approach for accountability: Putting state policies first. Association for the Study of Higher Education Forum.

Singleton, R. A. Jr., Straits, B. C. \& Straits, M. M. (1993). Approaches to social research, $2^{\text {nd }}$ ed. Oxford University Press.

Sizer, J., Spee, A. \& Bormans, R. (1992). The role of performance indicators in higher education. Higher Education, 24(2), 133-156.

Slaughter, S. \& Leslie, L. L. (1997). Academic capitalism: Politics, policies, and the entrepreneurial university. Baltimore and London: The Johns Hopkins University Press.

Slaughter, S. \& Rhoades, G. (2004). Academic capitalism and the new economy: Markets, state, and higher education. Baltimore and London: The John Hopkins University Press.

Smart, J. C. \& Hamm, R. E. (1993a). Organizational culture and effectiveness in twoyear colleges. Research in Higher Education, 34(4), 95-106.

Smart, J. C. \& Hamm, R. E. (1993b). Organizational effectiveness and mission orientation of two-year colleges. Research in Higher Education, 34(4), 489-502.

Smart, J. C. \& St. John, E. P. (1996). Organizational culture and effectiveness in higher education: A test of the "culture type" and "strong culture" hypotheses. Educational Evaluation and Policy Analysis, 18(3), 219-241. 
Steers, R. M. (1976). When is an organization effective? A process approach to understanding effectiveness. Organizational Dynamics, 5(2). 50-63.

Thelin, J. R. (2004). Higher education and the public trough: A historical perspective. In E. P. St. John \& M. D. Parsons (Eds.), Public funding of higher education: Changing contexts and new rationales. Baltimore and London: The Johns Hopkins University Press.

Tierney, W. G. (1988). Organizational culture in higher education: Defining the essentials. Journal of Higher Education, 59(1), 2-21.

Titus, M. A. (2006). No college student left behind: The influence of financial aspects of a state's higher education policy on college completion. The Review of Higher Education, 29(3), 293-317.

Trends in college pricing 2007. (2007). New York, NY: The College Board.

Trends in college spending: Where does the money come from? Where does it go? (2009). Delta Cost Project.

Tsui, A. S. (1990). A multiple-constituency model of effectiveness: An empirical examination at the human resource subunit level. Administrative Science Quarterly, $35(3), 458-483$.

Van de Ven, A. H. \& Ferry, D. L. (1980). Measuring and assessing organizations. New York: Wiley.

Wagner, A. (2009). Paradoxical life: Meaning, matter, and the power of human choice. Yale University Press.

Webster, D. S. (1981). Advantages and disadvantages of methods of assessing quality. 
Change, 13(7), 20-24.

Weick, K. E. (1976). Educational organizations as loosely coupled systems. Administrative Science Quarterly, 21, 1-19.

Welsh, J. F., \& Nunez, W. J. (2005). Faculty and administrative support for strategic planning: A comparison of two- and four-year institutions. Community College Review, 32(4), 20-39.

Whetten, D. A. (2004). In search of the $O$ in OMT. Distinguished Scholar Address, Organization and Management Theory Division, Academy of Management, New Orleans.

Winston, G. C. (1999). Subsidies, hierarchies and peers: the awkward economics of higher education. Journal of Economic Perspectives, 13, 13-36.

Yin, R. K. (2009). Case study research: Design and methods, $4^{\text {th }}$ ed. Sage Publications. Yuchtman, E. \& Seashore, S. E. (1967). A system resource approach to organizational effectiveness. American Sociological Review, 32(6), 891-903.

Zemsky, R. \& Wegner, G. R. (1997). Shaping the future. In P. M. Callan \& J. E. Finney (Eds.), Public and private financing of higher education: Shaping public policy for the future. American Council on Education: Oryx Press.

Zumeta, W. (2004). State higher education financing: Demand imperatives meet structural, cyclical, and political constraints. In E. P. St. John \& M. D. Parsons (Eds.), Public funding of higher education: Changing contexts and new rationales. Baltimore and London: The Johns Hopkins University Press. 


\title{
APENDIX A \\ E-mail Invitation to Participate in the Survey
}

Participate in the Effectiveness Survey

Dear,

A few days from now you will receive an e-mail asking you to fill out an on-line questionnaire about the educational outcomes, processes and work environment at $\mathrm{X}$ University.

The survey is part of the research for my doctoral dissertation. This research will help create a more complete understanding of factors influencing effectiveness of higher education institutions and is aimed to be a first step in building a model for sustainable higher education.

The subject line of the e-mail will be XU Effectiveness Survey and the e-mail will come from Mirela Blekic. Please take 10-15 minutes to fill out the survey when you see it in your inbox.

Thank you in advance for your time.

\author{
Mirela Blekic \\ Doctoral Candidate, Public Administration and Policy \\ Portland State University \\ 503-725-9892 \\ mirelab@pdx.edu
}


APENDIX A (continued)

Consent Document

(E-mail to complete the survey)

\section{Effectiveness Survey}

Dear ,

I am a doctoral student in the Hatfield School of Government, in the Department of Public Administration at Portland State University and my Dissertation Committee Chair is Dr. Craig Shinn. I am conducting a study about the effectiveness of higher education institutions and the role resources play in it. I would like to ask for your help in this study. The accompanying survey asks about your perceptions on educational outcomes, processes and work environment at X University and it should take 10-15 minutes to complete.

Your response is very important to me; the information you provide will help in understanding factors influencing effectiveness of higher education institutions and in building a model to assess effectiveness in higher education. Given a need for universities to respond to the demands of multiple constituencies but at the same time preserve their intrinsic values, developing a model to assess effectiveness is an important step in ensuring sustainability of higher education institutions.

Please take the survey now by following the link below:

(link here)

Your responses are confidential. Only summaries of total responses will be reported. No one will ever be able to identify you in any reports that are created from the data and no one who is not involved with the evaluation will ever see your answers. No identifiers beyond an e-mail address will be collected for respondents. The e-mail addresses will not be linked to survey responses and your contact information will be kept in a separate file from your responses. All data files will be stored in locked cabinets or on secure PSU servers.

Your participation in this survey is voluntary. Your willingness or unwillingness to participate will not affect your relationship with X University. Your completion of the survey indicates your consent to participate in this research. If you have any questions about the survey or wish to have your name removed from the mailing list, please contact Mirela Blekic at Portland State University (503-725-9892 or mirelab@pdx.edu).

If you have concerns or questions about this study, please contact the Human Subjects 
Research Review Committee, Office of Research and Sponsored Projects, Unitus Building 6th Floor, Portland State University, 503-725-4288, hsrrc@ pdx.edu.

Thank you for taking the time to complete this survey.

Sincerely,

Mirela Blekic

Doctoral Candidate, Public Administration and Policy

Portland State University 
APENDIX A (continued)

Follow-up E-mail to Complete the Survey

\author{
Effectiveness Survey \\ Dear ,
}

About a week ago, I e-mailed you a survey about the educational outcomes, processes and work environment at X University. If you have already responded to the survey, thank you. If not, please click on the survey link below at your earliest convenience, preferably within the next 5 days. I estimate that it will take approximately 10-15 minutes to complete the survey.

Please take the survey now by following the link below:

(link here)

Your responses are confidential. Only summaries of total responses will be reported. No one will ever be able to identify you in any reports that are created from the data and no one who is not involved with the evaluation will ever see your answers.

Participation in this survey is voluntary. Your willingness or unwillingness to participate will not affect your relationship with $X$ University. If you have any questions about the survey, or wish to have your name removed from the mailing list, please contact Mirela Blekic at Portland State University (503-725-9892 or mirelab@pdx.edu).

If you have concerns or questions about this study, please contact the Human Subjects Research Review Committee, Office of Research and Sponsored Projects, Unitus Building 6th Floor, Portland State University, 503-725-4288, hsrrc@pdx.edu.

Thank you for taking the time to complete this survey.

Sincerely, Mirela Blekic

Doctoral Candidate, Public Administration and Policy

Portland State University 


\section{APENDIX B}

Employee Survey

\section{Effectiveness Survey}

Thank you in advance for taking the time to complete this survey. Colleges and universities face an increased pressure to satisfy multiple and often competing demands, and the availability of resources is often tied to the perceived effectiveness of the institution as seen by various internal and external constituencies. This research examines organizational effectiveness in the higher education environment, its measurement and the role resources play in it.

This four-page survey asks a variety of questions about your perceptions related to processes and characteristics typical for this institution. The survey should take 10-15 minutes to complete. If you are not entirely sure how to answer a particular question, please provide your best estimate or leave the question blank.

Your responses are confidential and participation in this survey is voluntary. If you have questions about this survey, please contact Mirela Blekic at 503-725-9892 or mirelab@pdx.edu, or the Chair of the Human Subjects Research Review Committee in the Office of Research and Sponsored Projects at 503-725-4288 or hsrrc@pdx.edu.

\section{The items on the first two pages ask about the performance and actions of this institution.}

To what extent are the following characteristics typical of this institution?

\begin{tabular}{|c|c|c|c|c|c|c|}
\hline & Question & $\begin{array}{c}\text { Strongly } \\
\text { Agree }\end{array}$ & Agree & $\begin{array}{c}\text { Neither } \\
\text { Agree nor } \\
\text { Disagree }\end{array}$ & Disagree & $\begin{array}{l}\text { Strongly } \\
\text { Disagree }\end{array}$ \\
\hline 1.1 & $\begin{array}{l}\text { One of the outstanding } \\
\text { features of this institution is } \\
\text { the opportunity it provides } \\
\text { students for personal } \\
\text { development. }\end{array}$ & 0 & 0 & 0 & 0 & 0 \\
\hline 1.2 & $\begin{array}{l}\text { This institution is responsive } \\
\text { and adaptive to meeting the } \\
\text { changing needs of its } \\
\text { external constituencies, } \\
\text { which include individuals or } \\
\text { groups external to the } \\
\text { university but with links to } \\
\text { the university. }\end{array}$ & 0 & 0 & 0 & 0 & 0 \\
\hline
\end{tabular}




\begin{tabular}{|c|c|c|c|c|c|c|}
\hline 1.3 & $\begin{array}{l}\text { This institution has an ability } \\
\text { to obtain financial resources } \\
\text { in order to provide quality } \\
\text { instructional programs. }\end{array}$ & 0 & 0 & 0 & 0 & 0 \\
\hline 1.4 & $\begin{array}{l}\text { This institution has an ability } \\
\text { to obtain the resources it } \\
\text { needs to be effective. }\end{array}$ & 0 & 0 & 0 & 0 & 0 \\
\hline 1.5 & $\begin{array}{l}\text { When hiring new faculty } \\
\text { members, this institution can } \\
\text { attract leaders in their } \\
\text { respective fields. }\end{array}$ & 0 & 0 & 0 & 0 & 0 \\
\hline 1.6 & $\begin{array}{l}\text { This institution can attract } \\
\text { high achieving high school } \\
\text { graduates to attend. }\end{array}$ & 0 & 0 & 0 & 0 & 0 \\
\hline 1.7 & $\begin{array}{l}\text { After students leave this } \\
\text { institution, they maintain a } \\
\text { strong commitment to the } \\
\text { institution. }\end{array}$ & 0 & 0 & 0 & 0 & 0 \\
\hline 1.8 & $\begin{array}{l}\text { There is a feeling of } \\
\text { satisfaction among students } \\
\text { at this institution. }\end{array}$ & 0 & 0 & 0 & 0 & 0 \\
\hline 1.9 & $\begin{array}{l}\text { Many students drop out } \\
\text { because of dissatisfaction } \\
\text { with their educational } \\
\text { experiences at this } \\
\text { institution. }\end{array}$ & 0 & 0 & 0 & 0 & 0 \\
\hline 1.10 & $\begin{array}{l}\text { Many students complain } \\
\text { about their educational } \\
\text { experience at this institution } \\
\text { in venues such as, campus } \\
\text { newspaper, meetings with } \\
\text { faculty members or } \\
\text { administrators, or other } \\
\text { public forums. }\end{array}$ & 0 & 0 & 0 & 0 & 0 \\
\hline
\end{tabular}

\section{Please rate the graduates of this institution in the following:}




\begin{tabular}{|l|l|l|l|l|l|l|}
\hline \# & $\begin{array}{l}\text { These } \\
\text { graduates } \\
\text { are well } \\
\text { above } \\
\text { average }\end{array}$ & $\begin{array}{l}\text { These } \\
\text { graduates } \\
\text { are slightly } \\
\text { above } \\
\text { average }\end{array}$ & $\begin{array}{l}\text { These } \\
\text { graduates } \\
\text { are about } \\
\text { average }\end{array}$ & $\begin{array}{l}\text { These } \\
\text { graduates } \\
\text { are slightly } \\
\text { below } \\
\text { average }\end{array}$ & $\begin{array}{l}\text { These } \\
\text { graduates } \\
\text { are well } \\
\text { below } \\
\text { average }\end{array}$ \\
\hline $\begin{array}{l}\text { acquisition of } \\
\text { analytical, } \\
\text { problem-solving, } \\
\text { and } \\
\text { communication } \\
\text { skills. }\end{array}$ & 0 & 0 & 0 & 0 & 0 \\
\hline $\begin{array}{l}\text { Depth of } \\
\text { knowledge in their } \\
\text { major of study. }\end{array}$ & 0 & 0 & 0 & 0 & 0 \\
\hline $\begin{array}{l}\text { Community } \\
\text { engagement and } \\
\text { social } \\
\text { responsibility } \\
\text { development. }\end{array}$ & 0 & 0 & 0 & 0 & 0 \\
\hline
\end{tabular}

3. The following questions relate to employment satisfaction of institutional members. Please use the scale below in responding to these questions.

\begin{tabular}{|l|l|c|c|c|c|c|}
\hline 3 & Question & $\begin{array}{c}\text { A large } \\
\text { majority }\end{array}$ & $\begin{array}{c}\text { More } \\
\text { than } \\
\text { half }\end{array}$ & $\begin{array}{c}\text { About } \\
\text { half }\end{array}$ & $\begin{array}{c}\text { Less } \\
\text { than } \\
\text { half }\end{array}$ & $\begin{array}{c}\text { A small } \\
\text { minority }\end{array}$ \\
\hline 3.1 & $\begin{array}{l}\text { If given the chance of taking a } \\
\text { similar job at another institution, } \\
\text { how many faculty members do you } \\
\text { think would opt for leaving this } \\
\text { institution for professional } \\
\text { reasons? }\end{array}$ & 0 & 0 & 0 & 0 & 0 \\
$\begin{array}{l}\text { If given the chance of taking a } \\
\text { similar job at another institution, } \\
\text { how many administrators do you } \\
\text { think would opt for leaving this } \\
\text { institution for professional } \\
\text { reasons? }\end{array}$ & 0 & 0 & 0 & 0 & 0
\end{tabular}




\begin{tabular}{|c|c|c|c|c|c|c|}
\hline 3.3 & $\begin{array}{l}\text { If given the chance of taking a } \\
\text { similar job at another institution, } \\
\text { how many staff members do you } \\
\text { think would opt for leaving this } \\
\text { institution for professional } \\
\text { reasons? }\end{array}$ & 0 & 0 & 0 & 0 & 0 \\
\hline 3.4 & $\begin{array}{l}\text { Estimate how many faculty } \\
\text { members at this institution are } \\
\text { personally satisfied with their } \\
\text { employment. }\end{array}$ & 0 & 0 & 0 & 0 & 0 \\
\hline 3.5 & $\begin{array}{l}\text { Estimate how many administrators } \\
\text { at this institution are personally } \\
\text { satisfied with their employment. }\end{array}$ & 0 & 0 & 0 & 0 & 0 \\
\hline 3.6 & $\begin{array}{l}\text { Estimate how many staff members } \\
\text { at this institution are personally } \\
\text { satisfied with their employment. }\end{array}$ & 0 & 0 & 0 & 0 & 0 \\
\hline
\end{tabular}

4. To what extent are the following characteristics typical of this institution?

\begin{tabular}{|c|c|c|c|c|c|c|}
\hline$\#$ & Question & $\begin{array}{c}\text { Strongly } \\
\text { Agree }\end{array}$ & Agree & $\begin{array}{l}\text { Neither } \\
\text { Agree nor } \\
\text { Disagree }\end{array}$ & Disagree & $\begin{array}{l}\text { Strongly } \\
\text { Disagree }\end{array}$ \\
\hline 4.1 & $\begin{array}{l}\text { This institution emphasizes } \\
\text { activities outside the } \\
\text { classroom designed } \\
\text { specifically to enhance } \\
\text { students' personal, non- } \\
\text { academic development. }\end{array}$ & 0 & 0 & 0 & 0 & 0 \\
\hline 4.2 & $\begin{array}{l}\text { Students develop and mature } \\
\text { in non-academic areas (e.g., } \\
\text { socially, emotionally, } \\
\text { culturally) to a large degree } \\
\text { directly as a result of their } \\
\text { experiences at this institution. }\end{array}$ & 0 & 0 & 0 & 0 & 0 \\
\hline 4.3 & $\begin{array}{l}\text { This institution has the } \\
\text { reputation of possessing a } \\
\text { stimulating intellectual } \\
\text { environment with concern for } \\
\text { student academic } \\
\text { development. }\end{array}$ & 0 & 0 & 0 & 0 & 0 \\
\hline
\end{tabular}




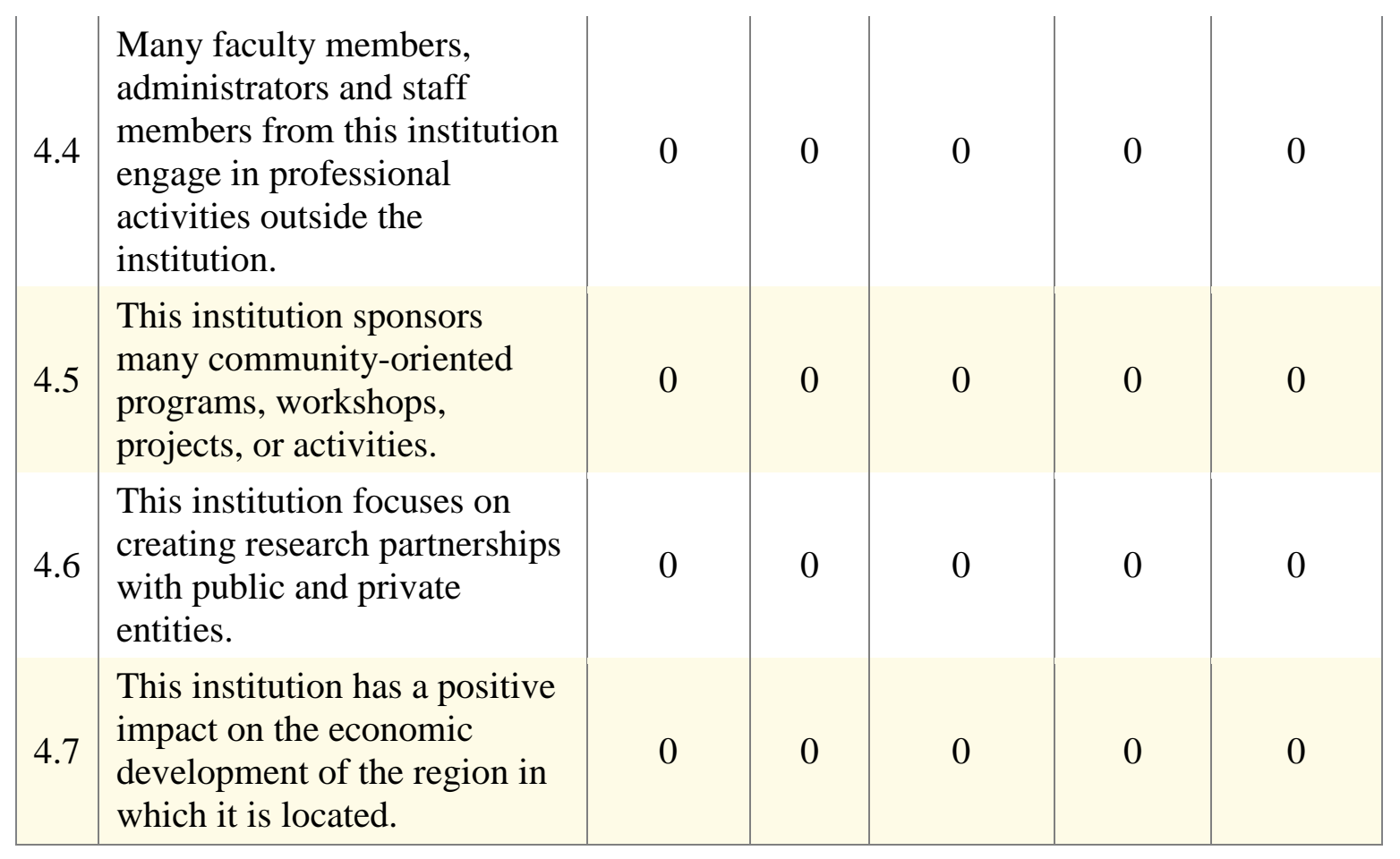

5. The following questions relate to the characteristics of faculty and students. Please use the scale below to respond.

\begin{tabular}{|l|l|c|c|c|c|}
\hline Question & $\begin{array}{c}\text { A large } \\
\text { majority }\end{array}$ & $\begin{array}{c}\text { More } \\
\text { than half }\end{array}$ & $\begin{array}{c}\text { About } \\
\text { half }\end{array}$ & $\begin{array}{c}\text { Less } \\
\text { than half }\end{array}$ & $\begin{array}{c}\text { A small } \\
\text { minority }\end{array}$ \\
\hline $\begin{array}{l}\text { How many faculty } \\
\text { members at this } \\
\text { institution would you } \\
\text { say published a book } \\
\text { or an article in a } \\
\text { professional journal, } \\
\text { or displayed a work of } \\
\text { art in a show last year? } \\
\text { What proportion of } \\
\text { the faculty members } \\
\text { would you estimate } \\
\text { teach at the "cutting } \\
\text { edge" of their field } \\
\text { i.e., require current } \\
\text { journal articles as } \\
\text { readings, revise } \\
\text { syllabi at least yearly, }\end{array}$ & 0 & 0 & 0 & 0 & 0 \\
\hline
\end{tabular}




\begin{tabular}{|c|c|c|c|c|c|c|}
\hline & $\begin{array}{l}\text { discuss current issues } \\
\text { in the field, etc.? }\end{array}$ & & & & & \\
\hline 5.3 & $\begin{array}{l}\text { How many faculty } \\
\text { members at this } \\
\text { institution are actively } \\
\text { engaged now in } \\
\text { professional } \\
\text { development activities } \\
\text { - e.g., doing research, } \\
\text { consulting, getting an } \\
\text { advanced degree, etc.? }\end{array}$ & 0 & 0 & 0 & 0 & 0 \\
\hline 5.4 & $\begin{array}{l}\text { How many faculty } \\
\text { members at this } \\
\text { institution would you } \\
\text { estimate have at some } \\
\text { time received an } \\
\text { academic award or } \\
\text { honor such as a } \\
\text { teaching, research, or } \\
\text { professional award or } \\
\text { a listing in a national } \\
\text { honorary directory? }\end{array}$ & 0 & 0 & 0 & 0 & 0 \\
\hline 5.5 & $\begin{array}{l}\text { How many faculty } \\
\text { members, } \\
\text { administrators, and } \\
\text { staff members at this } \\
\text { institution would you } \\
\text { say serve in the } \\
\text { community, in } \\
\text { government, on } \\
\text { boards and } \\
\text { committees, or in } \\
\text { other capacities? }\end{array}$ & 0 & 0 & 0 & 0 & 0 \\
\hline 5.6 & $\begin{array}{l}\text { In relation to other } \\
\text { institutions with which } \\
\text { it competes, what } \\
\text { proportion of the top } \\
\text { students attends this } \\
\text { institution rather than } \\
\text { the competition? }\end{array}$ & 0 & 0 & 0 & 0 & 0 \\
\hline
\end{tabular}


6. This section asks you to rate your perception of the general day-to-day

functioning of the overall institution. Please respond by choosing the number that best represents your perception of each item. If you agree strongly with one end of the scale, choose a number closer to that end of the scale. If you feel neutral about the item, choose a number near the middle of the scale. How do you perceive the following?

\begin{tabular}{|c|c|c|c|c|c|c|}
\hline \# & Question & Label 1 & $\begin{array}{l}\text { Label } \\
2\end{array}$ & $\begin{array}{l}\text { Label } \\
3\end{array}$ & $\begin{array}{l}\text { Label } \\
4\end{array}$ & Label 5 \\
\hline 6.1 & $\begin{array}{l}\text { Student/faculty } \\
\text { relationships }\end{array}$ & $\begin{array}{l}\text { Closeness, } \\
\text { lots of } \\
\text { informal } \\
\text { interactions, } \\
\text { mutual } \\
\text { personal } \\
\text { concern }\end{array}$ & 2 & 3 & 4 & $\begin{array}{l}\text { No closeness, } \\
\text { mostly } \\
\text { instrumental } \\
\text { relations, little } \\
\text { informal } \\
\text { interaction }\end{array}$ \\
\hline 6.2 & $\begin{array}{l}\text { Equity of treatment and } \\
\text { rewards }\end{array}$ & $\begin{array}{l}\text { People } \\
\text { treated fairly } \\
\text { and rewarded } \\
\text { equitably }\end{array}$ & 2 & 3 & 4 & $\begin{array}{l}\text { Favoritism and } \\
\text { inequity } \\
\text { present, unfair } \\
\text { treatment } \\
\text { exists }\end{array}$ \\
\hline 6.3 & $\begin{array}{l}\text { Interdepartmental } \\
\text { relations in the institution }\end{array}$ & $\begin{array}{l}\text { Lots of } \\
\text { coordination, } \\
\text { joint } \\
\text { planning } \\
\text { collaboration, } \\
\text { no friction }\end{array}$ & 2 & 3 & 4 & $\begin{array}{l}\text { No joint } \\
\text { activity, } \\
\text { conflict, lack } \\
\text { of coordination } \\
\text { and } \\
\text { communication }\end{array}$ \\
\hline 6.4 & $\begin{array}{l}\text { General pattern of } \\
\text { supervision and control }\end{array}$ & $\begin{array}{l}\text { Rigid } \\
\text { control, strict } \\
\text { supervision, } \\
\text { pressure for } \\
\text { conformity }\end{array}$ & 2 & 3 & 4 & $\begin{array}{l}\text { Respect for } \\
\text { differences, } \\
\text { personal } \\
\text { freedom, } \\
\text { individual } \\
\text { autonomy }\end{array}$ \\
\hline
\end{tabular}




\begin{tabular}{|c|c|c|c|c|c|c|}
\hline 6.5 & $\begin{array}{l}\text { Organizational health of } \\
\text { the institution }\end{array}$ & $\begin{array}{l}\text { Institution } \\
\text { runs } \\
\text { smoothly, } \\
\text { healthy } \\
\text { organization, } \\
\text { productive } \\
\text { internal } \\
\text { functioning }\end{array}$ & 2 & 3 & 4 & $\begin{array}{l}\text { Institution runs } \\
\text { poorly, } \\
\text { unhealthy } \\
\text { organization, } \\
\text { unproductive } \\
\text { internal } \\
\text { functioning }\end{array}$ \\
\hline 6.6 & $\begin{array}{l}\text { Recognition and reward } \\
\text { received for good work } \\
\text { from superiors }\end{array}$ & $\begin{array}{l}\text { Recognition } \\
\text { received for } \\
\text { good work, } \\
\text { rewarded for } \\
\text { success }\end{array}$ & 2 & 3 & 4 & $\begin{array}{l}\text { No rewards for } \\
\text { good work, no } \\
\text { one recognizes } \\
\text { success }\end{array}$ \\
\hline 6.7 & $\begin{array}{l}\text { The amount of } \\
\text { information or feedback } \\
\text { you receive }\end{array}$ & $\begin{array}{l}\text { Feel } \\
\text { informed, in- } \\
\text { the-know, } \\
\text { information } \\
\text { is available }\end{array}$ & 2 & 3 & 4 & $\begin{array}{l}\text { Feel isolated, } \\
\text { out-of-it, } \\
\text { information is } \\
\text { never available }\end{array}$ \\
\hline 6.8 & $\begin{array}{l}\text { The general social } \\
\text { environment }\end{array}$ & $\begin{array}{l}\text { Cooperative, } \\
\text { supportive, } \\
\text { mutual } \\
\text { concern for } \\
\text { others, } \\
\text { humane }\end{array}$ & 2 & 3 & 4 & $\begin{array}{l}\text { Competitive, } \\
\text { no support, } \\
\text { unsympathetic, } \\
\text { "every person } \\
\text { for } \\
\text { her/himself" }\end{array}$ \\
\hline
\end{tabular}

7. The next set of questions asks about activities and outcomes for this institution. Please use the scale below to respond.

\begin{tabular}{|l|l|c|c|c|c|c|}
$\#$ & $\begin{array}{c}\text { A large } \\
\text { majority }\end{array}$ & $\begin{array}{c}\text { More } \\
\text { than } \\
\text { half }\end{array}$ & $\begin{array}{c}\text { About } \\
\text { half }\end{array}$ & $\begin{array}{c}\text { Less } \\
\text { than } \\
\text { half }\end{array}$ & $\begin{array}{c}\text { A small } \\
\text { minority }\end{array}$ \\
\hline 7.1 & $\begin{array}{l}\text { How many students would you say } \\
\text { engage in extra academic work } \\
\text { (e.g., reading, studying, writing) } \\
\text { over and above what is specifically } \\
\text { assigned in the classroom? }\end{array}$ & 0 & 0 & 0 & 0 & 0 \\
$\begin{array}{l}\text { For how many graduates was } \\
\text { education received at this } \\
\text { institution beneficial in obtaining } \\
\text { employment? }\end{array}$ & 0 & 0 & 0 & 0 & 0
\end{tabular}




\begin{tabular}{|c|c|c|c|c|c|c|}
\hline 7.3 & $\begin{array}{l}\text { How many students who graduate } \\
\text { from this institution are well } \\
\text { prepared for challenges of today's } \\
\text { global economy? }\end{array}$ & 0 & 0 & 0 & 0 & 0 \\
\hline 7.4 & $\begin{array}{l}\text { How many graduates of this } \\
\text { institution are able to connect } \\
\text { classroom learning with real-world } \\
\text { experience? }\end{array}$ & 0 & 0 & 0 & 0 & 0 \\
\hline 7.5 & $\begin{array}{l}\text { How many students would you say } \\
\text { attend this institution to fulfill } \\
\text { career or occupational goals as } \\
\text { opposed to attending for social, } \\
\text { athletic, financial, or other reasons? }\end{array}$ & 0 & 0 & 0 & 0 & 0 \\
\hline
\end{tabular}

8. The following questions concern changes in conditions outside this institution over the last five years.

\begin{tabular}{|l|l|c|c|c|c|}
\hline Question & $\begin{array}{c}\text { Strongly } \\
\text { Agree }\end{array}$ & Agree & $\begin{array}{c}\text { Neither } \\
\text { Agree nor } \\
\text { Disagree }\end{array}$ & Disagree & $\begin{array}{c}\text { Strongly } \\
\text { Disagree }\end{array}$ \\
\hline 8.1 & $\begin{array}{l}\text { Factors outside the } \\
\text { institution that affect its } \\
\text { enrollments have become } \\
\text { more predictable over the } \\
\text { last five years. }\end{array}$ & 0 & 0 & 0 & 0 \\
$\begin{array}{l}\text { External uncertainties have } \\
\text { made institutional revenues } \\
\text { less predictable over the last } \\
\text { five years. } \\
\text { The number of potential } \\
\text { students from whom our } \\
\text { institution can recruit has } \\
\text { increased over the last five } \\
\text { years. }\end{array}$ & 0 & 0 & 0 & 0 \\
\hline $\begin{array}{l}\text { Financial resources have } \\
\text { become more difficult to } \\
\text { obtain over the last five } \\
\text { years. }\end{array}$ & 0 & 0 & 0 & 0 \\
\hline
\end{tabular}

9. The next section deals with the strategy this institution is pursuing. Please indicate the extent to which you agree or disagree with each item. 


\begin{tabular}{|c|c|c|c|c|c|c|}
\hline \# & Question & $\begin{array}{c}\text { Strongly } \\
\text { Agree }\end{array}$ & Agree & $\begin{array}{l}\text { Neither } \\
\text { Agree nor } \\
\text { Disagree }\end{array}$ & Disagree & $\begin{array}{l}\text { Strongly } \\
\text { Disagree }\end{array}$ \\
\hline 9.1 & $\begin{array}{l}\text { This institution is expanding } \\
\text { the array of academic } \\
\text { programs it offers. }\end{array}$ & 0 & 0 & 0 & 0 & 0 \\
\hline 9.2 & $\begin{array}{l}\text { This institution is increasing } \\
\text { the number of out-of-state } \\
\text { students it admits. }\end{array}$ & 0 & 0 & 0 & 0 & 0 \\
\hline 9.3 & $\begin{array}{l}\text { This institution is increasing } \\
\text { the investment in outreach } \\
\text { functions that deal with, for } \\
\text { example, admissions, } \\
\text { development, government } \\
\text { relations, businesses, non- } \\
\text { profits, and others. }\end{array}$ & 0 & 0 & 0 & 0 & 0 \\
\hline 9.4 & $\begin{array}{l}\text { This institution tries to } \\
\text { insulate itself from demands } \\
\text { and pressures in the } \\
\text { environment. }\end{array}$ & 0 & 0 & 0 & 0 & 0 \\
\hline 9.5 & $\begin{array}{l}\text { This institution is likely to be } \\
\text { the first to try new activities } \\
\text { or policies. }\end{array}$ & 0 & 0 & 0 & 0 & 0 \\
\hline 9.6 & $\begin{array}{l}\text { Institutional members educate } \\
\text { influential individuals and } \\
\text { groups about the value of the } \\
\text { institution. }\end{array}$ & 0 & 0 & 0 & 0 & 0 \\
\hline 9.7 & $\begin{array}{l}\text { This institution establishes } \\
\text { new domains of activity (e.g., } \\
\text { programs, initiatives). }\end{array}$ & 0 & 0 & 0 & 0 & 0 \\
\hline 9.8 & $\begin{array}{l}\text { Administrators at this } \\
\text { institution emphasize finding } \\
\text { new money for a balanced } \\
\text { budget. }\end{array}$ & 0 & 0 & 0 & 0 & 0 \\
\hline 9.9 & $\begin{array}{l}\text { Administrators at this } \\
\text { institution emphasize saving } \\
\text { money for a balanced budget. }\end{array}$ & 0 & 0 & 0 & 0 & 0 \\
\hline
\end{tabular}




\begin{tabular}{|c|c|c|c|c|c|c|}
\hline 9.10 & $\begin{array}{l}\text { Administrators at this } \\
\text { institution provide incentives } \\
\text { for conserving resources. }\end{array}$ & 0 & 0 & 0 & 0 & 0 \\
\hline
\end{tabular}

10. The last two questions ask about your position at this institution: What is your current position?
\# $\quad$ Answer
1 Administrator - Executive
2 Administrator - Academic Affairs
3 Administrator - Financial Affairs
4 Administrator - Student Affairs
5 Dean
6 Department Chair
7 Faculty
8 Professional Staff
9 Other (please specify)

11. What field, department, or program are you affiliated with?

\begin{tabular}{|c|l||}
\hline$\#$ & Answer \\
\hline 1 & Academic Affairs \\
2 & Agricultural Sciences \\
3 & Business Administration \\
4 & Computer Science \\
5 & Education \\
6 & Engineering \\
7 & Finance and Administration \\
8 & Fine and Performing Arts \\
9 & Government Relations \\
10 & Humanities \\
11 & Human Resources \\
12 & Information Technologies \\
13 & Law \\
14 & Library
\end{tabular}




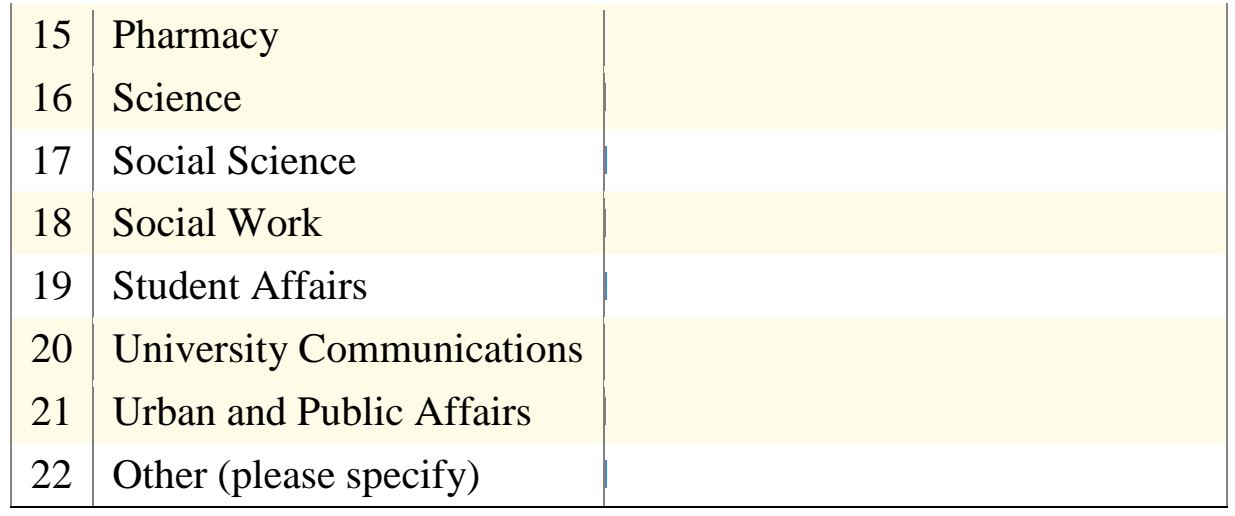

12. Do you have any other comment that you would like to share regarding your perceptions of the effectiveness of this institution? 


\title{
APPENDIX C
}

\author{
Student Survey
}

\section{Effectiveness Survey}

Thank you in advance for taking the time to complete this survey. Colleges and universities face an increased pressure to satisfy multiple and often competing demands, and the availability of resources is often tied to the perceived effectiveness of the institution as seen by various internal and external constituencies. This research examines organizational effectiveness in the higher education environment, its measurement and the role resources play in it.

This three-page survey asks a variety of questions about your perceptions related to processes and characteristics typical for this institution. The survey should take about 10 minutes to complete. If you are not entirely sure how to answer a particular question, please provide your best estimate or leave the question blank.

Your responses are confidential and participation in this survey is voluntary. If you have questions about this survey, please contact Mirela Blekic at 503-725-9892 or mirelab@pdx.edu, or the Chair of the Human Subjects Research Review Committee in the Office of Research and Sponsored Projects at 503-725-4288 or hsrrc@pdx.edu.

\section{To what extent are the following characteristics typical of this institution?}

\begin{tabular}{|c|c|c|c|c|c|c|}
\hline \# & Question & $\begin{array}{c}\text { Strongly } \\
\text { Agree }\end{array}$ & Agree & $\begin{array}{l}\text { Neither } \\
\text { Agree } \\
\text { nor } \\
\text { Disagree }\end{array}$ & Disagree & $\begin{array}{l}\text { Strongly } \\
\text { Disagree }\end{array}$ \\
\hline 1.1 & $\begin{array}{l}\text { One of the outstanding } \\
\text { features of this } \\
\text { institution is the } \\
\text { opportunity it provides } \\
\text { students for personal } \\
\text { development. }\end{array}$ & 0 & 0 & 0 & 0 & 0 \\
\hline 1.2 & $\begin{array}{l}\text { This institution is } \\
\text { responsive and adaptive } \\
\text { to meeting the changing } \\
\text { needs of its external } \\
\text { constituencies, which } \\
\text { include individuals or } \\
\text { groups external to the } \\
\text { university but with links } \\
\text { to the university. }\end{array}$ & 0 & 0 & 0 & 0 & 0 \\
\hline 1.3 & When hiring new & 0 & 0 & 0 & 0 & 0 \\
\hline
\end{tabular}




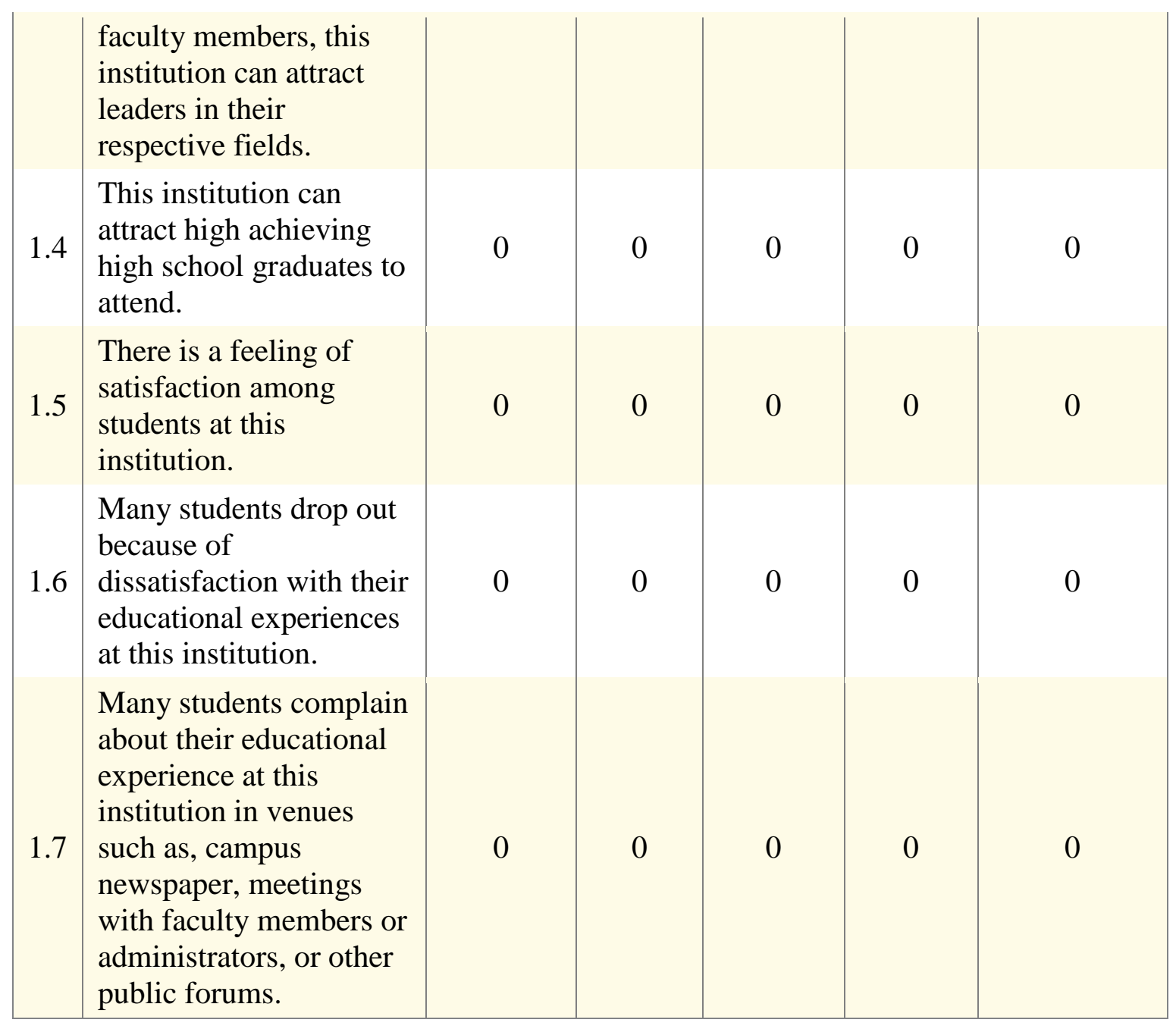

2. Please rate the graduates of this institution in the following:

\begin{tabular}{|l|l|l|l|l|l|l|}
\hline \# & $\begin{array}{l}\text { These } \\
\text { graduates } \\
\text { are well } \\
\text { above } \\
\text { average }\end{array}$ & $\begin{array}{l}\text { These } \\
\text { graduates } \\
\text { are slightly } \\
\text { above } \\
\text { average }\end{array}$ & $\begin{array}{l}\text { These } \\
\text { graduates } \\
\text { are about } \\
\text { average }\end{array}$ & $\begin{array}{l}\text { These } \\
\text { graduates } \\
\text { are slightly } \\
\text { below } \\
\text { average }\end{array}$ & $\begin{array}{l}\text { These } \\
\text { graduates } \\
\text { are well } \\
\text { below } \\
\text { average }\end{array}$ \\
\hline 2.1 & $\begin{array}{l}\text { Acquisition of } \\
\text { analytical, } \\
\text { problem-solving, } \\
\text { and } \\
\text { communication } \\
\text { skills. }\end{array}$ & 0 & 0 & 0 & 0 & 0
\end{tabular}




\begin{tabular}{|l|l|l|l|l|l|l|}
2.2 & $\begin{array}{l}\text { Depth of } \\
\text { knowledge in their } \\
\text { major of study. }\end{array}$ & 0 & 0 & 0 & 0 & 0 \\
\hline $\begin{array}{l}\text { Community } \\
\text { engagement and } \\
\text { social } \\
\text { responsibility } \\
\text { development. }\end{array}$ & 0 & 0 & 0 & 0 & 0 \\
\hline
\end{tabular}

3. To what extent are the following characteristics typical of this institution?

\begin{tabular}{|c|c|c|c|c|c|c|}
\hline \# & Question & $\begin{array}{c}\text { Strongly } \\
\text { Agree }\end{array}$ & Agree & $\begin{array}{l}\text { Neither } \\
\text { Agree nor } \\
\text { Disagree }\end{array}$ & Disagree & $\begin{array}{l}\text { Strongly } \\
\text { Disagree }\end{array}$ \\
\hline 3.1 & $\begin{array}{l}\text { This institution emphasizes } \\
\text { activities outside the } \\
\text { classroom designed } \\
\text { specifically to enhance } \\
\text { students' personal, non- } \\
\text { academic development. }\end{array}$ & 0 & 0 & 0 & 0 & 0 \\
\hline 3.2 & $\begin{array}{l}\text { This institution sponsors } \\
\text { many community-oriented } \\
\text { programs, workshops, } \\
\text { projects, or activities. }\end{array}$ & 0 & 0 & 0 & 0 & 0 \\
\hline 3.3 & $\begin{array}{l}\text { This institution has the } \\
\text { reputation of possessing a } \\
\text { stimulating intellectual } \\
\text { environment with concern for } \\
\text { student academic } \\
\text { development. }\end{array}$ & 0 & 0 & 0 & 0 & 0 \\
\hline 3.4 & $\begin{array}{l}\text { Students develop and mature } \\
\text { in non-academic areas (e.g., } \\
\text { socially, emotionally, } \\
\text { culturally) to a large degree } \\
\text { directly as a result of their } \\
\text { experiences at this institution. }\end{array}$ & 0 & 0 & 0 & 0 & 0 \\
\hline
\end{tabular}

4. The next two questions ask you to rate your perception of the general day-to-day functioning of the overall institution. If you agree strongly with one end of the scale, choose a number closer to that end of the scale. If you feel neutral about 
the item, choose a number near the middle of the scale. How do you perceive the following?

\begin{tabular}{|c|c|c|c|c|c|c|}
\hline \# & Question & Label 1 & $\begin{array}{l}\text { Label } \\
2\end{array}$ & $\begin{array}{l}\text { Label } \\
3\end{array}$ & $\begin{array}{l}\text { Label } \\
4\end{array}$ & Label 5 \\
\hline 4.2 & $x^{2}$ & $\begin{array}{l}\text { Closeness, } \\
\text { lots of } \\
\text { informal } \\
\text { interactions, } \\
\text { mutual } \\
\text { personal } \\
\text { concern } \\
\text { Institution } \\
\text { runs } \\
\text { smoothly, } \\
\text { healthy } \\
\text { organization, } \\
\text { productive } \\
\text { internal } \\
\text { functioning }\end{array}$ & 2 & 3 & 4 & $\begin{array}{l}\text { No } \\
\text { closeness, } \\
\text { mostly } \\
\text { instrumental } \\
\text { relations, } \\
\text { little } \\
\text { informal } \\
\text { interaction } \\
\text { Institution } \\
\text { runs poorly, } \\
\text { unhealthy } \\
\text { organization, } \\
\text { unproductive } \\
\text { internal } \\
\text { functioning }\end{array}$ \\
\hline
\end{tabular}

5. The next set of questions asks about activities and outcomes for this institution. Please use the scale below to respond.

\begin{tabular}{|l|l|c|c|c|c|}
\hline Question & $\begin{array}{c}\text { A large } \\
\text { majority }\end{array}$ & $\begin{array}{c}\text { More } \\
\text { than } \\
\text { half }\end{array}$ & $\begin{array}{c}\text { About } \\
\text { half }\end{array}$ & $\begin{array}{c}\text { Less } \\
\text { than } \\
\text { half }\end{array}$ & $\begin{array}{c}\text { A small } \\
\text { minority }\end{array}$ \\
\hline $\begin{array}{l}\text { How many faculty members, } \\
\text { administrators, and staff members at } \\
\text { this institution would you say serve } \\
\text { in the community, in government, on } \\
\text { boards and committees, or in other } \\
\text { capacities? }\end{array}$ & 0 & 0 & 0 & 0 & 0 \\
$\begin{array}{l}\text { How many students would you say } \\
\text { engage in extra academic work (e.g., } \\
\text { reading, studying, writing) over and } \\
\text { above what is specifically assigned } \\
\text { in the classroom? }\end{array}$ & 0 & 0 & 0 & 0 & 0 \\
\hline
\end{tabular}


For how many graduates was

5.3 education received at this institution beneficial in obtaining employment?

How many students who graduate from this institution are well prepared for challenges of today's global economy?

How many graduates of this institution are able to connect classroom learning with real-world experience?

How many students would you say attend this institution to fulfill career or occupational goals as opposed to attending for social, athletic, financial, or other reasons?

\begin{tabular}{l|l|l|l|l}
0 & 0 & 0 & 0 & 0 \\
0 & 0 & 0 & 0 & 0 \\
0 & 0 & 0 & 0 & 0 \\
0 & 0 & 0 & 0 & 0
\end{tabular}

\section{The next section deals with the strategy this institution is pursuing. Please indicate the extent to which you agree or disagree with each item.}

\begin{tabular}{|c|c|c|c|c|c|c|}
\hline \# & Question & $\begin{array}{c}\text { Strongly } \\
\text { Agree }\end{array}$ & Agree & $\begin{array}{l}\text { Neither } \\
\text { Agree nor } \\
\text { Disagree }\end{array}$ & Disagree & $\begin{array}{l}\text { Strongly } \\
\text { Disagree }\end{array}$ \\
\hline 6.1 & $\begin{array}{l}\text { This institution is expanding } \\
\text { the array of academic } \\
\text { programs it offers. }\end{array}$ & 0 & 0 & 0 & 0 & 0 \\
\hline 6.2 & $\begin{array}{l}\text { This institution is increasing } \\
\text { the number of out-of-state } \\
\text { students it admits. }\end{array}$ & 0 & 0 & 0 & 0 & 0 \\
\hline 6.3 & $\begin{array}{l}\text { This institution establishes } \\
\text { new domains of activity } \\
\text { (e.g., programs, initiatives). }\end{array}$ & 0 & 0 & 0 & 0 & 0 \\
\hline 6.4 & $\begin{array}{l}\text { Institutional members } \\
\text { educate influential } \\
\text { individuals and groups about } \\
\text { the value of the institution. }\end{array}$ & 0 & 0 & 0 & 0 & 0 \\
\hline 6.5 & $\begin{array}{l}\text { This institution has an ability } \\
\text { to obtain financial resources } \\
\text { in order to provide quality } \\
\text { instructional programs. }\end{array}$ & 0 & 0 & 0 & 0 & 0 \\
\hline
\end{tabular}


7. The last two questions ask about your history as a student at this institution: When you first enrolled to this institution you were a...

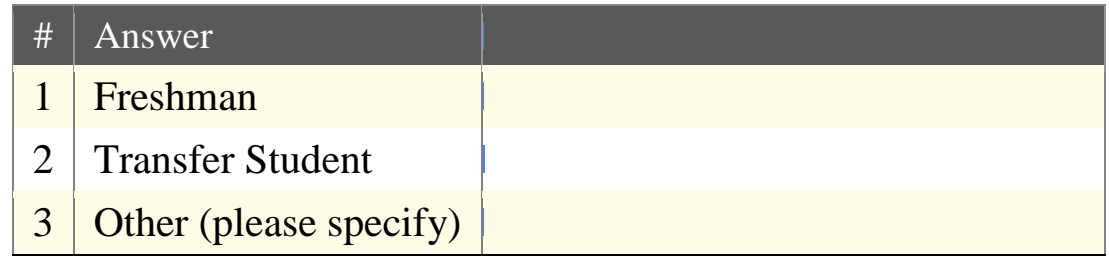

8. If you are a transfer student, please indicate your status at the time of transfer to this institution?

\begin{tabular}{|c|l|}
\hline \# & Answer \\
\hline 1 & Freshman \\
2 & Sophomore \\
3 & Junior \\
4 & Senior \\
5 & Other (please specify) \\
\hline
\end{tabular}

9. Do you have any other comment that you would like to share regarding your perceptions of the effectiveness of this institution? 


\section{APENDIX D \\ External Constituencies Survey}

\section{Effectiveness Survey}

Thank you in advance for taking the time to complete this survey. Colleges and universities face an increased pressure to satisfy multiple and often competing demands, and the availability of resources is often tied to the perceived effectiveness of the institution as seen by various internal and external constituencies. This research examines organizational effectiveness in the higher education environment, its measurement and the role resources play in it.

This three-page survey asks a variety of questions about your perceptions related to processes and characteristics typical for this institution. The survey should take 5-10 minutes to complete. If you are not entirely sure how to answer a particular question, please provide your best estimate or leave the question blank.

Your responses are confidential and participation in this survey is voluntary. If you have questions about this survey, please contact Mirela Blekic at 503-725-9892 or mirelab@pdx.edu, or the Chair of the Human Subjects Research Review Committee in the Office of Research and Sponsored Projects at 503-725-4288 or hsrrc@pdx.edu.

\section{To what extent are the following characteristics typical of this institution?}

\begin{tabular}{|c|c|c|c|c|c|c|}
\hline \# & Question & $\begin{array}{c}\text { Strongly } \\
\text { Agree }\end{array}$ & Agree & $\begin{array}{l}\text { Neither } \\
\text { Agree nor } \\
\text { Disagree }\end{array}$ & Disagree & $\begin{array}{l}\text { Strongly } \\
\text { Disagree }\end{array}$ \\
\hline 1.1 & $\begin{array}{l}\text { This institution is responsive } \\
\text { and adaptive to meeting the } \\
\text { changing needs of its external } \\
\text { constituencies, which include } \\
\text { individuals or groups external } \\
\text { to the university but with links } \\
\text { to the university. }\end{array}$ & 0 & 0 & 0 & 0 & 0 \\
\hline 1.2 & $\begin{array}{l}\text { This institution has an ability } \\
\text { to obtain financial resources } \\
\text { in order to provide quality } \\
\text { instructional programs. }\end{array}$ & 0 & 0 & 0 & 0 & 0 \\
\hline 1.3 & $\begin{array}{l}\text { This institution has an ability } \\
\text { to obtain the resources it } \\
\text { needs to be effective. }\end{array}$ & 0 & 0 & 0 & 0 & 0 \\
\hline
\end{tabular}


When hiring new faculty members, this institution can attract leaders in their respective fields.
0

\begin{tabular}{|l|l|l|l|}
0 & 0 & 0 & 0 \\
\hline
\end{tabular}

\section{Please rate the graduates of this institution in the following:}

\begin{tabular}{|l|l|l|l|l|l|l|}
\hline \# & Question & $\begin{array}{l}\text { These } \\
\text { graduates } \\
\text { are well } \\
\text { above } \\
\text { average }\end{array}$ & $\begin{array}{l}\text { These } \\
\text { graduates } \\
\text { are slightly } \\
\text { above } \\
\text { average }\end{array}$ & $\begin{array}{l}\text { These } \\
\text { graduates } \\
\text { are about } \\
\text { average }\end{array}$ & $\begin{array}{l}\text { These } \\
\text { graduates } \\
\text { are slightly } \\
\text { below } \\
\text { average }\end{array}$ & $\begin{array}{l}\text { These } \\
\text { graduates } \\
\text { are well } \\
\text { below } \\
\text { average }\end{array}$ \\
\hline 2.1 & $\begin{array}{l}\text { Acquisition of } \\
\text { analytical, } \\
\text { problem-solving, } \\
\text { and } \\
\text { communication } \\
\text { skills. } \\
\text { Depth of } \\
\text { knowledge in their } \\
\text { major of study. }\end{array}$ & 0 & 0 & 0 & 0 & 0 \\
\hline 2.3 & $\begin{array}{l}\text { Community } \\
\text { engagement and } \\
\text { social } \\
\text { responsibility } \\
\text { development. }\end{array}$ & 0 & 0 & 0 & 0 & 0 \\
\hline
\end{tabular}

\section{To what extent are the following characteristics typical of this institution?}

\begin{tabular}{|c|c|c|c|c|c|c|}
\hline \# & Question & $\begin{array}{c}\text { Strongly } \\
\text { Agree }\end{array}$ & Agree & $\begin{array}{l}\text { Neither } \\
\text { Agree nor } \\
\text { Disagree }\end{array}$ & Disagree & $\begin{array}{l}\text { Strongly } \\
\text { Disagree }\end{array}$ \\
\hline 3.1 & $\begin{array}{l}\text { This institution has the } \\
\text { reputation of possessing a } \\
\text { stimulating intellectual } \\
\text { environment with concern for } \\
\text { student academic } \\
\text { development. }\end{array}$ & 0 & 0 & 0 & 0 & 0 \\
\hline
\end{tabular}




\begin{tabular}{|c|c|c|c|c|c|c|}
\hline 3.2 & $\begin{array}{l}\text { Many faculty members, } \\
\text { administrators and staff } \\
\text { members from this institution } \\
\text { engage in professional } \\
\text { activities outside the } \\
\text { institution. }\end{array}$ & 0 & 0 & 0 & 0 & 0 \\
\hline 3.3 & $\begin{array}{l}\text { This institution sponsors } \\
\text { many community-oriented } \\
\text { programs, workshops, } \\
\text { projects, or activities. }\end{array}$ & 0 & 0 & 0 & 0 & 0 \\
\hline 3.4 & $\begin{array}{l}\text { This institution focuses on } \\
\text { creating research partnerships } \\
\text { with public and private } \\
\text { entities. }\end{array}$ & 0 & 0 & 0 & 0 & 0 \\
\hline 3.5 & $\begin{array}{l}\text { This institution has a positive } \\
\text { impact on the economic } \\
\text { development of the region in } \\
\text { which it is located. }\end{array}$ & 0 & 0 & 0 & 0 & 0 \\
\hline
\end{tabular}

4. The next set of questions asks about activities and outcomes for this institution. Please use the scale below to respond.

\begin{tabular}{|l|l|c|c|c|c|c|}
\hline$\#$ & $\begin{array}{c}\text { A large } \\
\text { majority }\end{array}$ & $\begin{array}{c}\text { More } \\
\text { than } \\
\text { half }\end{array}$ & $\begin{array}{c}\text { About } \\
\text { half }\end{array}$ & $\begin{array}{c}\text { Less } \\
\text { than } \\
\text { half }\end{array}$ & $\begin{array}{c}\text { A small } \\
\text { minority }\end{array}$ \\
\hline 4.1 & $\begin{array}{l}\text { How many faculty members, } \\
\text { administrators, and staff members at } \\
\text { this institution would you say serve } \\
\text { in the community, in government, } \\
\text { on boards and committees, or in } \\
\text { other capacities? }\end{array}$ & 0 & 0 & 0 & 0 & 0 \\
$\begin{array}{l}\text { For how many graduates was } \\
\text { education received at this institution } \\
\text { beneficial in obtaining employment? }\end{array}$ & 0 & 0 & 0 & 0 & 0 \\
$\begin{array}{l}\text { How many students who graduate } \\
\text { from this institution are well } \\
\text { prepared for challenges of today's } \\
\text { global economy? }\end{array}$ & 0 & 0 & 0 & 0 & 0
\end{tabular}


How many graduates of this institution are able to connect classroom learning with real-world experience?

How many students would you say attend this institution to fulfill career or occupational goals as opposed to attending for social, athletic, financial, or other reasons?

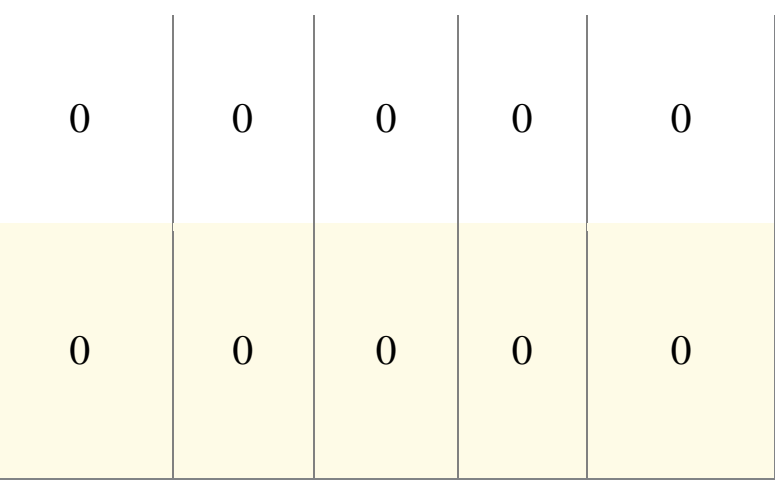

5. The following questions concern changes in conditions outside this institution over the last five years.

\begin{tabular}{|c|c|c|c|c|c|c|}
\hline \# & Question & $\begin{array}{l}\text { Strongly } \\
\text { Agree }\end{array}$ & Agree & $\begin{array}{l}\text { Neither } \\
\text { Agree nor } \\
\text { Disagree }\end{array}$ & Disagree & $\begin{array}{l}\text { Strongly } \\
\text { Disagree }\end{array}$ \\
\hline 5.1 & $\begin{array}{l}\text { External uncertainties have } \\
\text { made institutional revenues } \\
\text { less predictable over the } \\
\text { last five years. }\end{array}$ & 0 & 0 & 0 & 0 & 0 \\
\hline 5.2 & $\begin{array}{l}\text { Financial resources have } \\
\text { become more difficult to } \\
\text { obtain over the last five } \\
\text { years. }\end{array}$ & 0 & 0 & 0 & 0 & 0 \\
\hline
\end{tabular}

6. The next section deals with the strategy this institution is pursuing. Please indicate the extent to which you agree or disagree with each item.

\begin{tabular}{|c|c|c|c|c|c|c|}
\hline \# & Question & $\begin{array}{c}\text { Strongly } \\
\text { Agree }\end{array}$ & Agree & $\begin{array}{l}\text { Neither } \\
\text { Agree nor } \\
\text { Disagree }\end{array}$ & Disagree & $\begin{array}{l}\text { Strongly } \\
\text { Disagree }\end{array}$ \\
\hline 6.1 & $\begin{array}{l}\text { This institution is expanding } \\
\text { the array of academic } \\
\text { programs it offers. }\end{array}$ & 0 & 0 & 0 & 0 & 0 \\
\hline 6.2 & $\begin{array}{l}\text { This institution is increasing } \\
\text { the number of out-of-state } \\
\text { students it admits. }\end{array}$ & 0 & 0 & 0 & 0 & 0 \\
\hline
\end{tabular}




\begin{tabular}{|c|c|c|c|c|c|c|}
\hline 6.3 & $\begin{array}{l}\text { This institution is increasing } \\
\text { the investment in outreach } \\
\text { functions that deal with, for } \\
\text { example, admissions, } \\
\text { development, government } \\
\text { relations, businesses, non- } \\
\text { profits, and others. }\end{array}$ & 0 & 0 & 0 & 0 & 0 \\
\hline 6.4 & $\begin{array}{l}\text { This institution tries to } \\
\text { insulate itself from demands } \\
\text { and pressures in the } \\
\text { environment. }\end{array}$ & 0 & 0 & 0 & 0 & 0 \\
\hline 6.5 & $\begin{array}{l}\text { This institution is likely to be } \\
\text { the first to try new activities } \\
\text { or policies. }\end{array}$ & 0 & 0 & 0 & 0 & 0 \\
\hline 6.6 & $\begin{array}{l}\text { Institutional members educate } \\
\text { influential individuals and } \\
\text { groups about the value of the } \\
\text { institution. }\end{array}$ & 0 & 0 & 0 & 0 & 0 \\
\hline 6.7 & $\begin{array}{l}\text { This institution establishes } \\
\text { new domains of activity (e.g., } \\
\text { programs, initiatives). }\end{array}$ & 0 & 0 & 0 & 0 & 0 \\
\hline 6.8 & $\begin{array}{l}\text { Administrators at this } \\
\text { institution emphasize finding } \\
\text { new money for a balanced } \\
\text { budget. }\end{array}$ & 0 & 0 & 0 & 0 & 0 \\
\hline 6.9 & $\begin{array}{l}\text { Administrators at this } \\
\text { institution emphasize saving } \\
\text { money for a balanced budget. }\end{array}$ & 0 & 0 & 0 & 0 & 0 \\
\hline
\end{tabular}

7. The last two questions ask about your relationship with this institution: What is your current affiliation with this institution?

\section{Answer}

1 Board of Higher Education Member

2 Business Community Member

3 Local Government Representative

4 Non-profit Sector Member

5 State Legislator

6 Other (please specify) 
8. If you are a business, local government or non-profit, please indicate your current position?

\begin{tabular}{|l|l|l|}
\hline \# & Answer \\
\hline 1 & Executive & \\
2 & Manager/Supervisor & \\
3 & Human Resources Representative & \\
4 & Other (please specify) & \\
\hline
\end{tabular}

9. Do you have any other comment that you would like to share regarding your perceptions of the effectiveness of this institution? 


\section{APENDIX E}

\section{Survey Key}

Dimensions of Effectiveness:

1 - Student educational satisfaction

2 - Student academic and personal development

3 - Student career development

4 - Capacity to maintain or expand resource base

5 - Faculty, administrator and staff employment satisfaction

6 - Professional development and quality of the faculty

7 - System openness and interaction

8 - Resources for quality programs, faculty and students

9 - Organizational culture and health

10 - Ability to acquire, save and use resources effectively

11- Ability to overcome financial difficulties

$\mathrm{x}$ - Employees

* - Students

**. External Constituencies

\section{TABLE 28}

Relationship Between Survey Items and Dimensions of Effectiveness

\begin{tabular}{|c|c|c|c|c|c|c|c|c|c|c|c|}
\hline \multicolumn{12}{|c|}{ Effectiveness Dimension } \\
\hline Survey & & & & & & & & & & & \\
\hline Item & 1 & 2 & 3 & 4 & 5 & 6 & 7 & 8 & 9 & 10 & 11 \\
\hline 1.1 & & $\mathrm{x}^{*}$ & & & & & & & & & \\
\hline 1.2 & & & & & & & $\mathrm{x} * * *$ & & & & \\
\hline 1.3 & & & & & & & & $\mathrm{X} * * *$ & & & \\
\hline 1.4 & & & & & & & & $\mathrm{x} * *$ & & & \\
\hline 1.5 & & & & & & & & $\mathrm{X} * * *$ & & & \\
\hline 1.6 & & & & & & & & $\mathrm{x}^{*}$ & & & \\
\hline 1.7 & & & & & & & & $\mathrm{x}$ & & & \\
\hline 1.8 & $\mathrm{x}^{*}$ & & & & & & & & & & \\
\hline 1.9 & $\mathrm{X}^{*}$ & & & & & & & & & & \\
\hline 1.10 & $\mathrm{X}^{*}$ & & & & & & & & & & \\
\hline 2.1 & & $\mathrm{x} * * *$ & & & & & & & & & \\
\hline 2.2 & & $\mathrm{x}^{* * * *}$ & & & & & & & & & \\
\hline 2.3 & & $\mathrm{x} * * *$ & & & & & & & & & \\
\hline 3.1 & & & & & $\mathrm{x}$ & & & & & & \\
\hline 3.2 & & & & & $\mathrm{x}$ & & & & & & \\
\hline
\end{tabular}




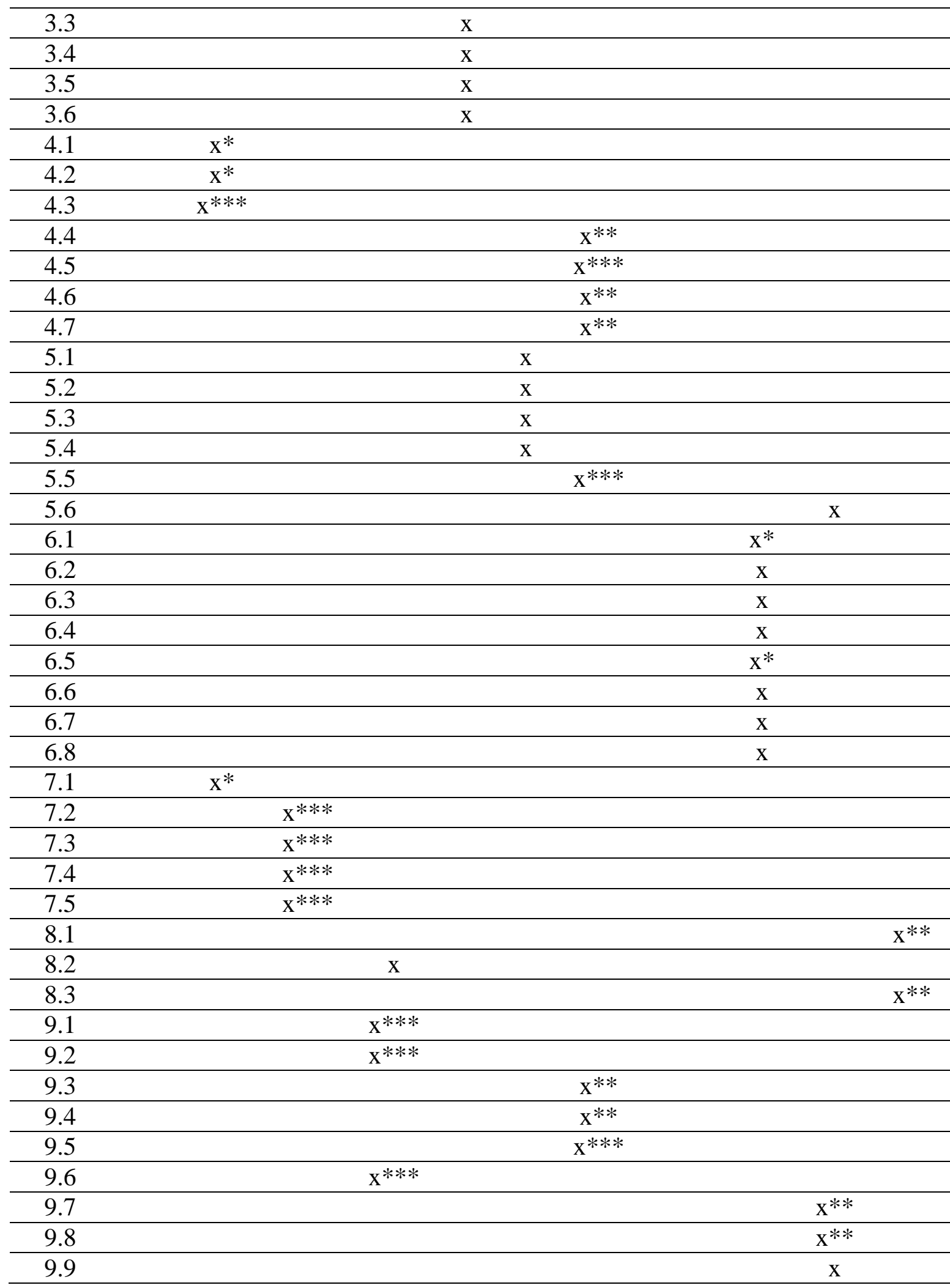




\section{APPENDIX F \\ COMPILATION OF RESPONSES TO OPEN-ENDED QUESTION}

Question: Do you have any other comment that you would like to share regarding your perceptions of the effectiveness of this institution?

- "While there are improvements in the university's image in the community, internal operational issues are not as yet. What I generally feel is the failure of all educational institutions is helping students who graduate find jobs. The real measure of a student success is to gain a job in his/her field after obtaining a hard earned, expensive, degree." - "Too many students come to this university solely to get a job. This means that many students do not show an interest or commitment to academic, campus, or community engagement."

- "This university needs serious organizational help, from the top down. Inefficiencies in practices and policies are everywhere and there aren't enough staff or resources to combat the root causes. We spend too much time trying to navigate idiosyncratic and outdated university policies. We should be spending this time with our students, engaging them in the community so they'll go out in the world and be ambassadors for University. I spend more time getting around the system because it doesn't work than I should ever have to. Things like registration and scheduling never work. Have you heard of another large university that does paper registration? Simple things like that would make the small tasks easier for faculty, which then allows us to be more effective in the classroom. University practices are unsustainable, which is so ironic it's not funny. Why does each department buy their own paper from Staples or wherever? Why isn't the university 
buying paper in bulk? We could get more sustainable paper for less money if someone was in charge of sourcing that across the university. Why did the university cut the two printer technicians a few years ago? Those two people fixed all the printers on campus. Now if our printer breaks we spend \$200 on having an outside contractor come look at it, and that doesn't include repairs. \$200 for a visit. How many printers break on campus every day? It's usually easier to throw them out than to get them fixed. And how does that work in with the mission statement of the university. I think faculty would be more inclined to actively get on the sustainability bandwagon if we didn't see such stupid waste happening every day. Then you'd have active participation by the majority, rather than frantic promotion by a very small minority. The university needs leadership, and frankly that's not going to come from the old guard administration that leads us. President is a good leader, but it doesn't work to have one person lead an organization."

- "This institution has forgotten that students are not merely people in search of an education, but consumers engaging in an expensive undertaking and as such have rights when the institution fails to provide its promised curriculum. In overlooking its obligation to provide all that it advertises and collects student funds for this institution has done its students numerous disservices."

- "This was a strange survey to take. It was long, and I completed it in a time I felt pressure to be doing something else. I also don't think this survey gets at what is happening in terms of higher education increasingly becoming a gatekeeper in terms of people who need education not being able to afford it and having increased challenge in improving their social location.” 
- "This institution has a serious lack in senior leadership when it comes to allocating resources and strategic planning. Furthermore, resources are not allocated based on need/productivity. Peter is robbed to pay Paul; structural problems are ignored. This carries over to garnering outside resources through development. University has no simple articulated message or brand. We add positions to perform a function before we underwrite the positions we already have that serve university-wide. By any measure our infrastructure is broken; we have no clear articulated vision."

- "I don't think people are that happy in this institution. But I believe people stay because of the institution's location - the area has a lot to offer, and because of the unemployment rate and financial crisis. There isn't a perception and maybe even a reality that people within higher education can be as mobile as was possible previously."

- "There are many questions relating to the need for resources yet University Relations is not listed under field, department or program. I have found this omission to be consistent and it reflects a major obstacle to our ability to secure private funding. There is limited awareness of the need and a resulting lack of integration of the message in campus wide activities and efforts.”

- "The question "This institution has an ability to obtain financial resources in order to provide quality instructional programs." I agree that they have the ability to do so if they privatize which I do not value as an adequate way of obtaining financial resources. Therefore I disagree that University has an ability to obtain financial resources creatively."

- "The faculty feel completely devalued by the administration." 
- "The effectiveness of this institution remains high, and will become compromised with continued tuition cost, primarily, due in part that Financial Aid cannot meet the demands of increasing tuition, living cost and fees associated with the high number of enrolling students."

- "Sorry to be so vague in my responses. I know little about the University or its faculty. We have hired 2 grads from this University for an accounting/bookkeeping position. Both have been well versed and outstanding employees."

- "The University is clustered with internal politics to the point that the left hand does not know what the right hand is doing. I frequently hear arguments between staff. Also, I am not so surprised that most of my instructors don't have a firm grasp of the subject matter they are teaching. In addition, I have seen University pass over highly qualified professors in favor of "new blood. The University has a reputation of a college to go to if you can't afford to move out of state or out of city, to a better University. I am only here because I love the city and think the city is a great place to live. The Art Department is a joke! Having been here for about a year now, I have learned less than I did at the community college. And that is not saying much I sympathize with the instructors who are given a job to do, but find themselves with their hands tied. Too many adjunct and part time instructors. I called one of mine today, only to find out that she did not have voicemail for me to leave an important message. There is talent at University/Art. Some of the instructors have a lot to offer. Health center is a great idea. It is too bad that it does not function as well as it should. Out of all the appointments that I have made there, resolution has been found perhaps half of the time. Over all, I would say that University 
needs some serious overhauling and perhaps a "common sense" approach to the day to day execution of the school."

- "The University has served my needs well. I would recommend it to anyone interested in a quality education for a somewhat affordable price. Students only get out of their education, what they put in."

- "The University has been referred to by alums as "chaos on the blocks" - meaning, lack of coherent systems, policies, and lack of collaborative depts and divisions. The amt of "towers" and "Turf" that are present here is extraordinary - I have never worked at a place that interacts less with itself."

- "The University serves as a valuable asset to the community by facilitating the higher education of individuals who also actively engage in the working society, instead of isolating themselves in college towns and student housing. This allows the insight into how their education will serve them as a functional member of society as they are cultivating themselves in said society, as opposed to the imagined ideals of many other college students in the state and the U.S. who find themselves in a manner of culture shock when they leave their universities for the real world."

- "People get out of this University what they put into it. People expect things to be handed to them but miss the fact that they are not making the most of their educational experience and not connecting with faculty and making important connections."

- "Overall effectiveness is good, but improvement could be made in requirement of graduates. Additional communications classes, both written and spoken should be required. There appears to be a degradation over the past decade in the ability of 
graduates to spell, use proper grammar and communicate effectively in the business environment. Additionally, there is still a disconnect between theory and application once in the work force. One solution is to require internships in their desired field of labor in order to graduate. That is somewhat onerous, but would help alleviate the disconnect. In my experience, a 4.0 student rarely translates into a great employee. It just means that they know how to get good grades, but don't necessarily know how to be a good employee."

- "Over all the University is a pleasure to work for. The students, Faculty, Staff everyone. The job that I do is fun and I enjoy coming to work each day."

- "Our school is highly effective, an exciting place to be, and on the rise in terms of reputation. We are engaged in the community to an extraordinary degree, head and shoulders above more prestigious institutions. However we are not prestigious, our faculty, staff, and administrator salaries are painfully low in comparison to comparator institutions. There is a contrast between our actual value (which is high) and how we are perceived by the general public, by the legislature, and by potential students (which is lower). Also, our department and several others are nationally recognized ... there is variance across departments on student attitude and professional recognition." - "My perceptions may be biased by being in a strong department. I think we prepare our students very well and contribute strongly to the economic health of the region. This is particularly true because of the access we give to place-bound and older students." - "My perception is largely based on my experiences with the undergraduate students I have worked with. While they are not lacking I tend to find students from some of the 
other local universities stand out more than students from this university. I felt unable to comment on many of the questions you posed."

- "I m not sure that many people in the city know about the effectiveness of this institution - which is why you're doing this survey I imagine and I think a good idea." - "I have recruited and hired outstanding new employees through the employment office."

- "I think we have a fairly strong faculty given the status of the university and the compensation it offers. Generally our students are average or above with a few that are quite good. In some of our graduate programs, we are attracting the highest ranked students and competing with the most elite schools. Faculty are not very well rewarded nor is there much institutional support but perhaps those days are over for all universities. I think we have a good president who understands the university and is actively engaged in promoting it and finding a path in these difficult times. We definitely need to do more for students in terms of connecting them to occupational opportunities and developing in them a sense of identity, loyalty and long term connection to the university."

- "I think that the students we interview from this University are well prepared academically, but that they are not as well prepared as students from other universities in the state when it comes to presenting themselves in the career marketplace. (I have most knowledge of students from the business school in particular.). While the career services office at the business school tries its best and does the most possible with what it has, these efforts have not been supported enough by the institution in terms of resources. Moreover, it does not appear that the faculty and administration act in partnership with 
career services to provide the tools students need to present themselves as top candidates. It seems they expect the one person left in the business school's career services office to be solely responsible for students' career preparation while the rest of the institution is disconnected from that effort when, in fact, they should be much more intertwined. This is something other universities in the state do much better than this University."

- "I have no opinion/perception for several of the questions on the survey. I strongly believe, however, that this University does far more community based work than your average state institution."

- "I attend part-time in the evenings. I think the experience for a student like myself problem varies widely from the experiences of other more traditional students."

- "I am disappointed with the lack of academic rigor at this university. To put it bluntly, an "A" is easy to get here."

-"How can one even justify the comment of "This institution is expanding the array of academic programs it offers" when University is offering less than ever, has a constant staff of rotating adjuncts and hardly any programs of study. Much has been cut even in the last two years that I have attended. I am an English major at the senior level and there were four English classes for me to choose from this term. Four. I am both appalled and disappointed with the University especially with the way it allocates funds. It's ridiculous that we're paying more than ever and getting less than ever."

- "A resource poor university that keeps trying to more than it can do well. Always more students to fit in, always seeking more acclaim by starting some new trendy program, but always doing so on the backs of underfunded programs, and overworked faculty. The 
University always says it values faculty, but really values only dollars. Each time a contract is negotiated, the university fights as hard as it possibly can to cut back salaries and benefits while class sizes grow and administrative support never does."

- "As a relative newcomer to the University and to higher education, I have been struck by how difficult it is to get anything done here and by how the institution is managed not by outcomes or by productivity, but by traditions and politics. I still feel that University has the potential to be the most significant contributor to prosperity and well-being in our region (the reason I came here), but I feel like the administration - from the very top down - has been flat-footed in responding to the new economic reality and has spent too much time on ill-planned nice-to-haves, instead of focusing on those things that will be crucial to our region's future."

- "The questions posed by this survey are subjective, when objective numbers are available. This will not be a responsible dataset."

- "Wealthy people should not control how a college is run."

- "Undergraduate research made the difference for me. I wish more students of all majors had the same opportunities."

- "Tuition is far too high for what they offer in return."

- "Too much money goes to sports. Need more funding for science, math programs."

- "This is an incredible institution, but administration often shows a disregard for the perspectives and opinions of students as a major stakeholder group." 
- "This institution lacks high quality communication and enthusiasm between teachers and their students which at times cause the quality of the learning experience to be extremely degraded."

- "There is lots of focus on incoming freshmen but much less for transfer and particular non-traditional students. “

- "The University has improved dramatically over the 20 years that I have been here; better students, better faculty, better facilities, etc."

- "The pressures on public education at all levels in the state will only be increasing in the coming years. It's not clear to me how my institution (or its peers) will respond to these pressures, which made completing this survey somewhat difficult."

- "The faculty at this institution are much better than the institution deserves. Pay is at about $70 \%$ of the AVERAGE pay for comparable faculty at comparable institutions. Faculty are, all things considered, incredibly dedicated to their students and to delivering an education on the level of the educations we got at (almost invariably) more prestigious institutions. The crappiness of this university, in terms of institutional support and even washing of windows (once a decade, whether they need it or not) is endemic. The imbalance between athletic spending and spending on our core mission (uh... education?) is a constant insult. I have applied several times to other positions, but since I've been aiming particularly high (I was shortlisted at $\mathrm{X}$ University a few years ago, and at $\mathrm{Y}$ University more recently) I've not yet left, but I will continue to apply as the more attractive positions come up. University's attitude ("it's good enough : )) is not good enough." 
- "It's obvious to me that the University only cares about wealthy young people and their schedules. As an older, non-traditional student it is extremely difficult to complete my degree and work a job because they only teach upper level classes between the hours o 9 AM and 5 PM. If I need an evening class, it's not available. And there's scant little to choose from as an online class, that gives me a more flexible schedule. The University needs to care about people who are not so wealthy that they don't have to work wile going to school. It's clear that those are the University's top priority."

- "Institution has been instrumental in structuring new funding model across academic departments that allows departments to actually plan more effectively. Emphasis on interdisciplinary programs has grown and has gained momentum which is a win for everyone."

- "Increasingly the institution is suffering from a widening rift between administration and faculty. The values of the two groups are drifting further apart. There is ever less communication and respect on both sides."

- "In terms of academic education, this institution doesn't seem any more capable than the community college I came from, where I paid half as much.”

- "I think the institution is fairly effective, with plenty of room for growth. I'm encouraged by the quality of new hires and new students. The trajectory is good. This survey is a little challenging with respect to this institution: doesn't reflect the right disciplines, doesn't understand how our employees are described (what is staff? classified employees?). It probably should have been tested more thoroughly before using it." 
- "I am sorry but I do not have much experience with this institution so I could not answer very many of your questions."

- "Having already obtained a Bachelor's degree (that I have been unable to transfer into a profession and/or income [not from this institution]) I may be jaded as to the effectiveness of most liberal arts degrees in their relationship to obtaining employment. Most four year degrees, [in my opinion] unless directly related to some sort of professional certificate, e.g. nursing or teaching, do not translate directly into a means of employment. And this is something I think is almost hidden by all post-secondary institutions. I was always told that a degree meant a higher wage and thus a higher quality of life, whatever the degree happens to be. But this institution, like most I know of are not truthful in letting people know that they will have 30 years of student loans and no guarantee of a higher wage, if any wage at all. I think this is a huge disservice down to students."

- "I love the focus on athletics, but I wish the tutoring center for the entire rest of the student population was better than being stuck in the basement of one of the oldest buildings on campus. Also, the Honors College is stuck in the upstairs of a building, when it should be paid more attention to. 